\title{
BROOKHEVEN
}

NATIONAL LABORATORY

\section{THE NEW YORK MIDTOWN DISPERSION STUDY (MID-05) METEOROLOGICAL DATA}

\author{
R. Michael Reynolds, T.M. Sullivan, S. Smith, V. Cassella
}

July 25, 2006

\section{Environmental Sciences Department/Environmental Research \& Technology Division}

\author{
Brookhaven National Laboratory \\ P.O. Box 5000 \\ Upton, NY 11973-5000 \\ www.bnl.gov
}

\begin{abstract}
Notice: This manuscript has been authored by employees of Brookhaven Science Associates, LLC under Contract No. DE-AC02-98CH10886 with the U.S. Department of Energy. The publisher by accepting the manuscript for publication acknowledges that the United States Government retains a non-exclusive, paid-up, irrevocable, world-wide license to publish or reproduce the published form of this manuscript, or allow others to do so, for United States Government purposes.
\end{abstract}




\section{DISCLAIMER}

This report was prepared as an account of work sponsored by an agency of the United States Government. Neither the United States Government nor any agency thereof, nor any of their employees, nor any of their contractors, subcontractors, or their employees, makes any warranty, express or implied, or assumes any legal liability or responsibility for the accuracy, completeness, or any third party's use or the results of such use of any information, apparatus, product, or process disclosed, or represents that its use would not infringe privately owned rights. Reference herein to any specific commercial product, process, or service by trade name, trademark, manufacturer, or otherwise, does not necessarily constitute or imply its endorsement, recommendation, or favoring by the United States Government or any agency thereof or its contractors or subcontractors. The views and opinions of authors expressed herein do not necessarily state or reflect those of the United States Government or any agency thereof. 


\title{
THE NEW YORK MIDTOWN DISPERSION STUDY (MID-05) METEOROLOGICAL DATA REPORT
}

\author{
July 25, 2006
}

R. Michael Reynolds

T.M. Sullivan

S. Smith

V. Cassella

Brookhaven National Laboratory

\begin{abstract}
The New York City midtown dispersion program, MID05, examined atmospheric transport in the deep urban canyons near Rockefeller Center. Little is known about air flow and hazardous gas dispersion under such conditions, since previous urban field experiments have focused on small to medium sized cities with much smaller street canyons and examined response over a much larger area. During August, 2005, a series of six gas tracer tests were conducted and sampling was conducted over a $2 \mathrm{~km}$ grid. A critical component of understanding gas movement in these studies is detailed wind and meteorological information in the study zone. .To support data interpretation and modeling, several meteorological stations were installed at street level and on roof tops in Manhattan. In addition, meteorological data from airports and other weather instrumentation around New York City were collected. This document describes the meteorological component of the project and provides an outline of data file formats for the different instruments. These data provide enough detail to support highly-resolved computational simulations of gas transport in the study zone.
\end{abstract}




\section{Table of Contents}

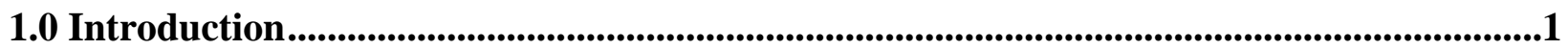

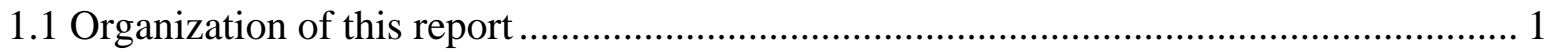

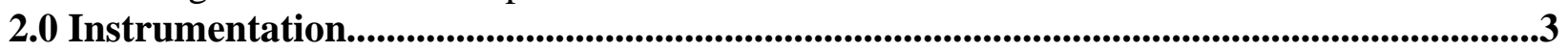

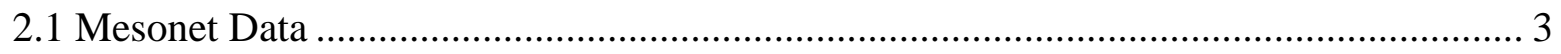

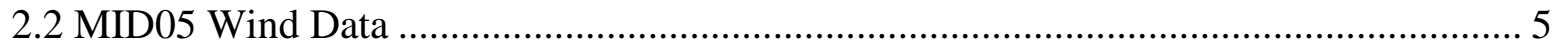

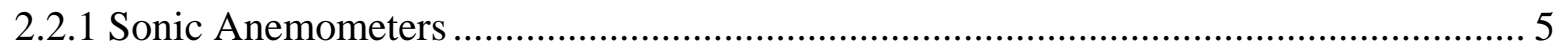

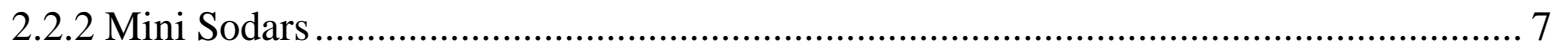

3.0 Instrumentation Location and Data File Identification ......................................................10

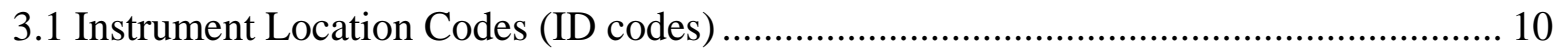

4.0 Deployment ...............................................................................................................................16

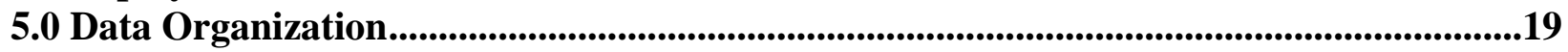

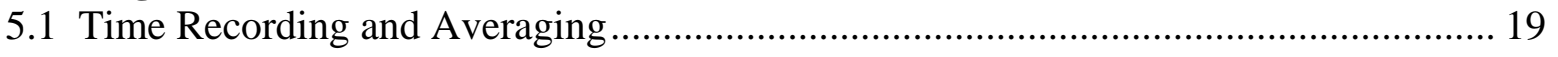

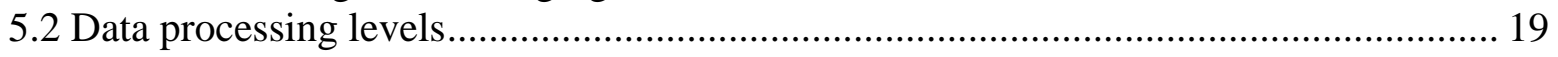

5.3 Level 1 Data File Naming Convention ................................................................ 22

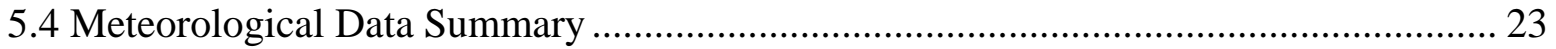

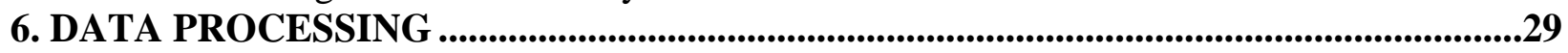

6.1 Sonic Anemometer Data Processing................................................................... 29

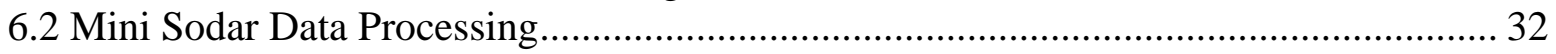

7. Equipment Quality Assurance Tests................................................................................37

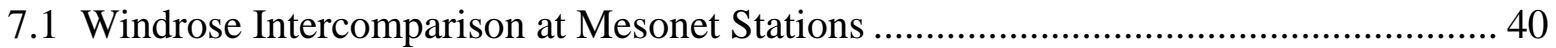

7.2 Wind Intercomparisons in the BNL Meteorological Field ...................................... 44

Appendix A: Listing of All Folders and Files In Level 0 for MID05 ...................................59

Appendix B: Listing of All Folders and Files for Level 1 Data ..................................................74

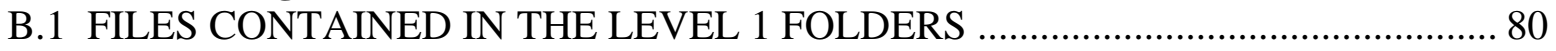

Appendix C: RM Young 81000 UltraSonic Anemometer ..................................................83 


\section{List of Figures}

Figure 1 Roof top installation of 3D sonic anemometer.

Figure 2 Mini Sodar on the MetLife Building with 3DSonic anemometer in the left background.

Figure 3 Photographs of the twelve street locations for sonic anemometers used in MID05 tests.

Figure 4 Map showing locations of roof top stations deployed for the MID05 experiment. ...... 14 Figure 5 Map showing the locations of street sonic anemometer stations during IOPs $1-3$ and IOPs 4 - 6.

Figure 6 The mini Sodar and wind measurements from the MetLife building roof................... 39 Figure 7 The mini Sodar and wind measurements from the General Motors Building (gm) roof.

40

Figure 8 Directional Wind Rose from nine mesonet stations in August 2005. ........................ 42 Figure 9 The vector speed, vector direction, and air temperature at $10 \mathrm{~m}$ (blue) and $88 \mathrm{~m}$ (red) from the BNL meteorological tower.......................................................................... 44 Figure 10 Wind speed and direction measured by the mini Sodar at $20 \mathrm{~m}$ (blue) and $100 \mathrm{~m}$ (red).

Figure 11 Comparsion of Wind Speed and Direction for the miniSodar at 20, 40, 60, and 90 meters and the BNL meteorological tower at $88 \mathrm{~m}$.

Figure 12 The group mean wind speed (left column) and direction (right column) during each of

the intercomparison periods.

Figure 13 Comparison of measured wind direction to the group mean wind direction for instruments SN 320, SN 364, and SN 607 in group 1. 


\section{List of Tables}

Table 1 Specifications and Settings for 3D sonic anemometers during MID05.......................... 5

Table 2 Settings for Mini Sodars used during MID05 ...................................................... 8

Table 3 Station identification codes, location and geographic locaters .................................. 10

Table 4 Station identification codes and level 0 File Folder name........................................... 20

Table 5. Summary of data collected by each sonic anemometer station for each day of the experiment. The numbers are in Mbytes of raw data for each day. Note that a full day of $10-\mathrm{Hz}$ data produces a file of 58 Mbytes.................................................................................... 24

Table 6 Street level 3D anemometer operating times (EST) ............................................. 24

Table 7 Roof top and set back 3D anemomtor operating times during the IOPs. ..................... 26

Table 8 Meteorological variables measured at the mesonet stations........................................ 27

Table 9 File format for 3D sonic anemometer data processed and averaged over a 5 minute

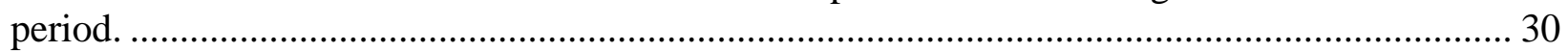

Table 10 mini Sodar data table ....................................................................................... 33

Table 11 Results from the three-step cleaning process for SODAR wind time series from

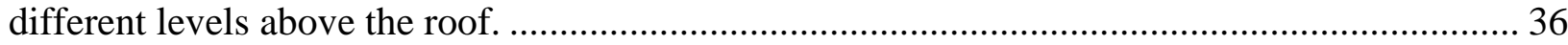

Table 12 The mean bias and standard deviation for each sonic anemometer during the four-part

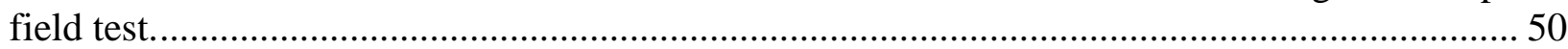




\subsection{Introduction}

In August 2005 a series of six tracer studies were conducted in near Rockefeller Center as part of the New York City Urban Dispersion Program (UDP). Collectively these studies are known as MID05 they examined outdoor releases of Perfluorocarbon Tracer (PFT) tracers and Sulfur hexafluoride $\left(\mathrm{SF}_{6}\right)$, indoor releases of PFTs, and subway releases of PFTs. Depending on the test, up to 160 samplers were used to measure $\mathrm{SF}_{6}$ and up to 80 samplers were used to measure PFTs during the sampling event. Samplers were located covering a $2 \mathrm{~km}$ square grid as well as being in the building being tested and along several subway lines. A critical component of understanding gas movement in these studies is detailed wind and meteorological information.

This document provides details on the meteorological equipment used and data that were collected by Brookhaven National Laboratory (BNL) during the UDP, MID05. Intense Operation Periods (IOPs) were conducted on August .8, 12, 14, 18, 20 and 24 in which portable meteorological instrumentation was deployed and operated from 6A.M. to 12 A.M., Eastern Standard Time (EST). In addition, a mesonet was formed using local area airports and other installed weather instrumentation as well as roof top instrumentation installed specifically for the UDP tests. Mesonet data were collected continuously from at least a week prior to the first IOP and for several weeks after the last IOP. This report focuses on data collected during the sampling period of August 8th - 24th.

\subsection{Organization of this report}

Instrumentation: The instrumentation is described in a general way in Section 2. 
Locations: Location sites are named and described in Section 3. The location sites are described independently from the instrumentation located there. Thus a wind station and a mini Sodar can occupy the same location, for example a roof top on a building.

Deployment procedures: Section 4 describes the procedures followed in deploying, aligning, and data recording.

Data Organization: The structure of the data files from the different instruments is discussed in section 5 .

Data Processing: Section 6 provides review of the data processing methods used for the wind and sodar data sets.

Quality Assurance: Section 7 reviews several techniques used for quality assurance. Tests were performed at the BNL meteorology field to determine the quality of the sodar and 3D sonic anemometer data. 


\subsection{Instrumentation}

Several different types of weather instrumentation were available for data collection during the MID05 study period. Data from surrounding weather stations at airports, Universities, and National Oceanic and Atmospheric Administrations (NOAA) National Weather Service (NWS) stations were available. This data is referred to as the mesonet for these experiments. The mesonet stations are described briefly in this section and the data collected is described in Section 5. In addition, mini Sodars and sonic anemometers were installed on rooftops and building setbacks for the duration of the test period. Sonic anemometers were also installed at street level during the IOPs. The data format for these instruments is described in Appendix A and $\mathrm{B}$.

\subsection{Mesonet Data}

Mesonet data was collected from several locations. General information on the different types of stations is provided below. Data collected and stored as part of the MID05 experiment are discussed in chapter 5 and Appendix B of this report.

\subsubsection{ASOS Stations}

The Automated Surface Observing Systems (ASOS) program is a joint effort of the National Weather Service (NWS), the Federal Aviation Administration (FAA), and the Department of Defense (DOD). The ASOS systems serve as the nation's primary surface weather observing network. ASOS is designed to support weather forecast activities and aviation operations and, at the same time, support the needs of the meteorological, hydrological, and climatological research communities. ASOS stations report basic weather elements including:

- Sky condition: cloud height and amount (clear, scattered, broken, overcast) up to 12,000 feet - Visibility: (to at least 10 statute miles) 
- Basic present weather information: type and intensity for rain, snow, and freezing rain

- Obstructions to vision: fog, haze

- Pressure: sea-level pressure, altimeter setting

- Ambient temperature, dew point temperature

- Wind direction, speed and character (gusts, squalls)

- Precipitation accumulation

- Selected significant remarks including- variable cloud height, variable visibility, precipitation beginning/ending times, rapid pressure changes, pressure change tendency, wind shift, peak wind.

More information on these sites can be found at http://www.nws.noaa.gov/asos/

\subsubsection{City College New York (CCNY) Meteorological Station}

The NOAA-CREST weather station, located on the roof of the Marshak Science Building on

Convent Avenue north of $135^{\text {th }}$ Street in Manhattan, began operation on 12 December, 2003. The station's sensors collect data each second and compute one-minute averages. The station's sensor array collects data in one-minute cycles controlled by the program resident in a datalogger at the site. The datalogger is polled and the information downloaded to the host computer in the Steinman Hall Engineering Building via the campus internet. Archived data is available on the NOAA-CREST web site. Data includes wind speed, direction and their vectors; air, dew point, wet bulb, heat index and wind chill temperatures; relative humidity; air pressure; rain; plant trans-evaporation and solar flux. More information on this weather station can be found at http://icerd.engr.ccny.cuny.edu/noaa/wc/

\subsubsection{Stevens Institute of Technology}

The New York Harbor Observing and Prediction System (NYHOPS) was established to permit an assessment of ocean, weather, environmental, and vessel traffic conditions throughout the New York Harbor region. The system is designed to provide a knowledge of meteorological and oceanographic conditions both in real-time and forecasted out to 48 hours in the Hudson River, 
the East River, NY/NJ Estuary, Raritan Bay, Long Island Sound and the coastal waters of New Jersey. For more information see http://hudson.dl.stevens-tech.edu/NYHOPS/

\section{NOAA DCNET/URBANet}

DCNet/URBANet is a NOAA program designed to test and evaluate the feasibility of acquiring wind turbulence measurements within the complex topology of the urban environment. They maintain weather instrumentation to provide three-dimensional wind speed and direction, relative humidity, temperature and other derived weather variables. In New York City they maintain stations at the Environmental Measurement Laboratory (EML) and Times Square. More information can be found at http://dcnet.atdd.noaa.gov/.

\subsection{MID05 Wind Data}

Data collected specifically for the MID05 tests included street level sonic anemometers and roof top level anemometers and mini Sodars.

\subsubsection{Sonic Anemometers}

The three-dimensional sonic anemometer (3Dsonic) model 81000 manufactured by R.M. Young Co. was used for turbulence measurements in this project. The instrument provided velocity measurements in 3 dimensions ( $\mathrm{u}, \mathrm{v}$, and $\mathrm{w}$ ) and sonic temperature (Tsonic). The instrument brochure with all specifications is given in Appendix C. Table 1, below, provides key characteristics for the measurements.

Table 1 Specifications and Settings for 3D sonic anemometers during MID05

\begin{tabular}{|l|l|}
\hline Variable & Settings \\
\hline Internal sampling rate & $160 \mathrm{~Hz}$ \\
\hline
\end{tabular}




\begin{tabular}{|l|l|}
\hline Output sample rate & $10 \mathrm{~Hz}$ \\
\hline Output format & ASCII \\
\hline Output variables & $\mathrm{u}, \mathrm{v}, \mathrm{w}$, Tsonic \\
\hline Wind speed range & $+/-40 \mathrm{~m} / \mathrm{s}$ \\
\hline Wind speed resolution & $\pm 0.01 \mathrm{~m} / \mathrm{s}$ \\
\hline Valid wind elevation angles & $\pm 60 \mathrm{deg}$ \\
\hline
\end{tabular}

Sonic anemometers are well suited for urban locations, especially at street level, where wind speeds have considerable vertical flow and where wind velocities can be quite small. However, a limitation in this type of instrument is that measurements become suspect with the elevation from horizontal exceeds $\pm 60^{\circ}$. 3Dsonic data where this limitation is exceeded is flagged in the data file. The ASCII digital output from the 3Dsonic is collected by a laptop PC running a customized program called "ECCheck.” 


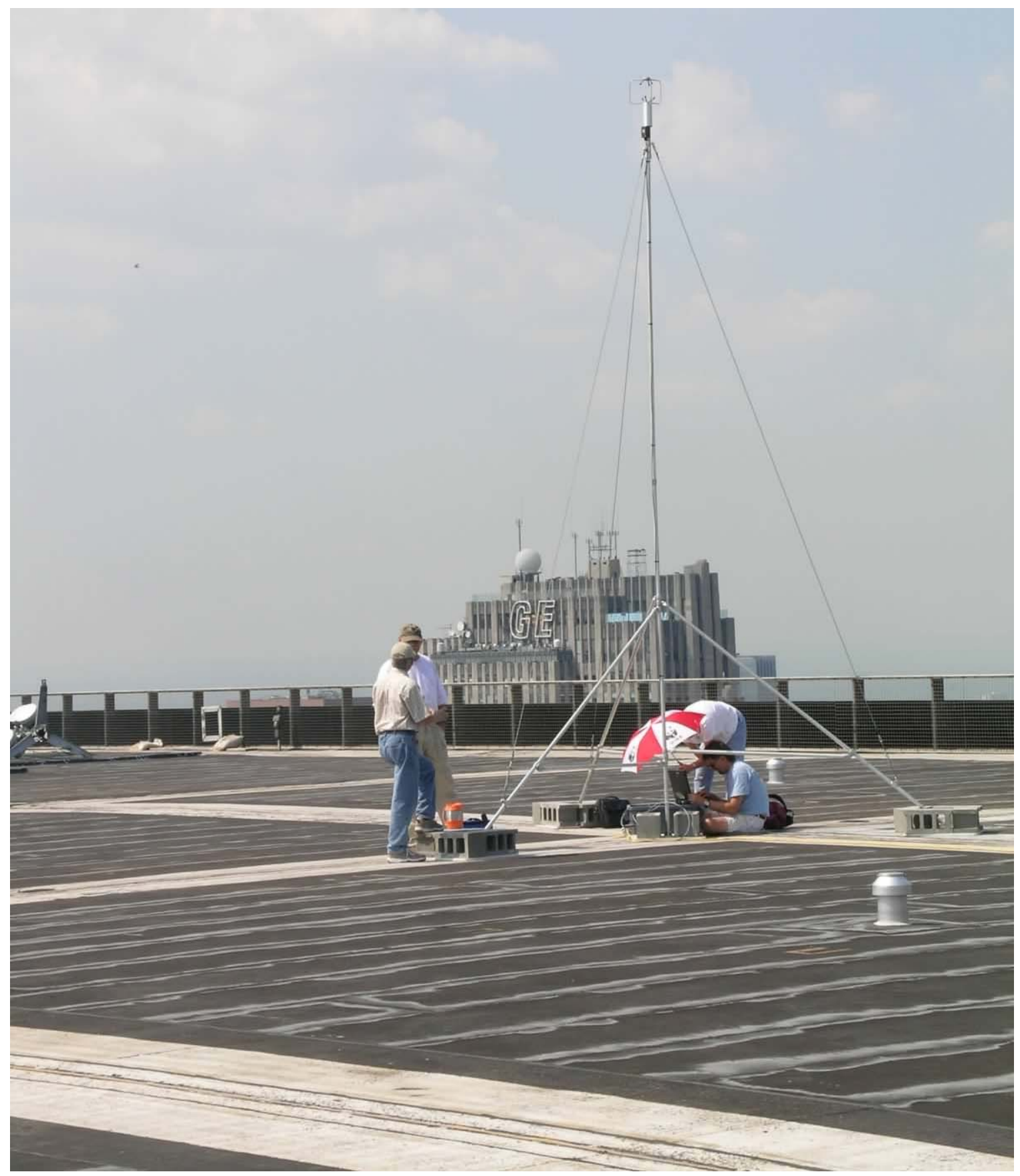

Figure 1 Roof top installation of 3D sonic anemometer.

\subsubsection{Mini Sodars}

Sodar (sonic detection and ranging) systems were used to remotely measure the vertical turbulence structure and the wind profile of the lower layer of the atmosphere. Sodars operate on 
the principle of acoustic backscattering. The mini Sodar, the instrument used in this study, was developed by AeroVironment Inc. in the late 1980’s to measure wind profiles in helicopter landing areas where noise levels were extreme. It was made commercially available in 1994 and has been deployed in situations where winds within the lowest $200 \mathrm{~m}$ are needed and ambient noise in the lower frequency range (1000-2000 Hz) is high. It was determined that the optimum frequency to operate Sodar in these conditions was near $4500 \mathrm{~Hz}$. The same conclusion was reached by Crescenti and Baxter (1998) and Crescenti (1998) for the urban environment where automobiles and HVAC systems can add considerable ambient noise. The following table lists the specifications for the mini Sodar. Figure 2 shows a picture of a mini Sodar installed on the roof of the MetLife building.

Table 2 Settings for Mini Sodars used during MID05

\begin{tabular}{|l|c|}
\hline \multicolumn{1}{|c|}{ Parameter } & Settings \\
\hline Maximum Sampling Altitude & $200 \mathrm{~m}$ \\
\hline Minimum sampling Altitude & $15 \mathrm{~m}$ \\
\hline Height Resolution & $10 \mathrm{~m}$ \\
\hline Transmit frequency & $4500 \mathrm{~Hz}$ \\
\hline Averaging interval & $2 \mathrm{~min}$ \\
\hline Wind speed range & $0-35 \mathrm{~m} / \mathrm{s}$ \\
\hline Wind speed accuracy & $<0.2 \mathrm{~m} / \mathrm{s}$ \\
\hline Wind direction accuracy & $<5 \mathrm{deg}$ \\
\hline Power input (electrical) & $30 \mathrm{~W}$ \\
\hline Power output (acoustic avg) & $40 \mathrm{~W}$ \\
\hline Voltage & $120 / 220 \mathrm{VAC}$ \\
\hline Weight & $255 \mathrm{lbs}$ \\
\hline Antenna ht & $1.2 \mathrm{~m}$ \\
\hline Antenna Width & $1.2 \mathrm{~m}$ \\
\hline Antenna length & $1.5 \mathrm{~m}$ \\
\hline Total ht with collar & $2.1 \mathrm{~m}$ \\
\hline Beam width & 16 \\
\hline Beam zenith angles & $6 \mathrm{deg}$ \\
\hline
\end{tabular}




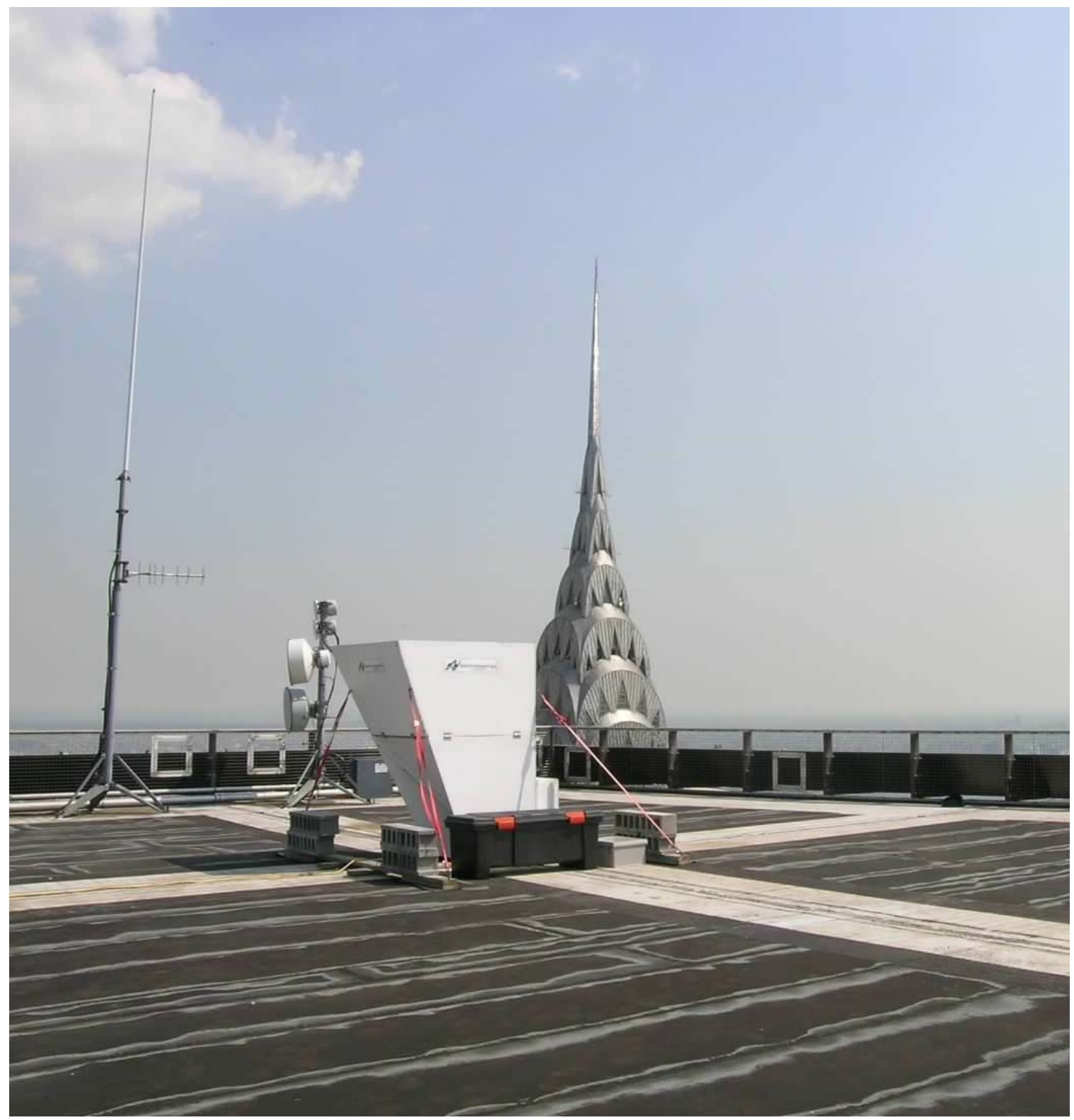

Figure 2 Mini Sodar on the MetLife Building with 3DSonic anemometer in the left background. 


\subsection{Instrumentation Location and Data File Identification}

\subsection{Instrument Location Codes (ID codes)}

A set of ID codes has been developed in order to provide unambiguous reference to any meteorological data in the project. Table 3 presents the station ID codes, a narrative description of the location, latitude, longitude, ground elevation in meters above mean sea level (MSL), and instrument elevation in meters above ground level. Latitude and longitude values are based on the WGS 84 datum.

Table 3 Station identification codes, location and geographic locaters

\begin{tabular}{|l|l|l|l|l|c|}
\hline \multicolumn{1}{|c|}{$\begin{array}{c}\text { Station } \\
\text { Identifier }\end{array}$} & \multicolumn{1}{|c|}{ Location } & Latitude & Longitude & $\begin{array}{c}\text { Ground } \\
\text { Elevation } \\
(\mathrm{m})(\mathrm{msl})\end{array}$ & $\begin{array}{c}\text { Wind } \\
\text { sensor } \\
\text { Elevation } \\
(\mathrm{m})\end{array}$ \\
\hline \multicolumn{5}{|c|}{ Regional Meteorological Stations } \\
\hline cpk & $\begin{array}{l}\text { Central park, NOAA } \\
\text { ASOS }\end{array}$ & 40.7833 & -73.967 & 39.6 & 25 \\
\hline ewr & Newark Airport ASOS & 40.7167 & -74.1667 & 2.3 & 10 \\
\hline field & $\begin{array}{l}\text { BNL meteorological } \\
\text { field }\end{array}$ & 40.87083 & -72.6167 & 27.4 & 3 \\
\hline fok & $\begin{array}{l}\text { Westhampton Gabreski } \\
\text { Airport }\end{array}$ & 40.85 & -72.6333 & 20.4 & 10 \\
\hline hpn & $\begin{array}{l}\text { White Plains } \\
\text { Westchester Co Airport }\end{array}$ & 41.0667 & -73.7 & 115.5 & 10 \\
\hline hwv & $\begin{array}{l}\text { Shirley Brookhaven } \\
\text { Airport }\end{array}$ & 40.8167 & -72.8667 & 25 & 10 \\
\hline isp & $\begin{array}{l}\text { Islip Long Island } \\
\text { Macarthur Airport }\end{array}$ & 40.8 & -73.1 & 25.6 & 10 \\
\hline lga & $\begin{array}{l}\text { New York LaGuardia } \\
\text { Airport }\end{array}$ & 40.7833 & -73.8833 & 3.4 & 10 \\
\hline jfk & $\begin{array}{l}\text { New York J F Kennedy } \\
\text { Int'l Airport }\end{array}$ & 40.6333 & -73.7667 & 3.4 & 10 \\
\hline & \multicolumn{1}{|c|}{ Roof top meteorological stations } & & \\
\hline
\end{tabular}




\begin{tabular}{|c|c|c|c|c|c|}
\hline ccny & $\begin{array}{l}\text { CCNY Roof, NOAA } \\
\text { GPS station }\end{array}$ & 40.8192 & -73.95 & 98 & 58 \\
\hline eml & $\begin{array}{l}\text { Environ Meas Lab, 15th } \\
\text { floor, roof }\end{array}$ & 40.72842 & -74.0066 & 5 & 82 \\
\hline gm1 & $\begin{array}{l}\text { GM Building Roof } \\
\text { (3D sonic anemometer) }\end{array}$ & 40.76357 & -73.9725 & -- & 225 \\
\hline gm2 & $\begin{array}{l}\text { GM Building Roof } \\
\text { (mini Sodar) }\end{array}$ & 40.76352 & -73.9724 & - - & 225 \\
\hline lbr1 & $\begin{array}{l}\text { Bldg A, } 745 \text { Seventh } \\
\text { Ave, Roof installation }\end{array}$ & dcnet & dcnet & - - & -- \\
\hline lbr2 & $\begin{array}{l}\text { Bldg A, S side setback, } \\
\text { above 49th St. }\end{array}$ & 40.76041 & -73.9833 & -- & $\sim 32$ \\
\hline met1 & $\begin{array}{l}\text { MetLife Building } \\
\text { (3D sonic anemometer) }\end{array}$ & 40.75342 & -73.9765 & -- & 247 \\
\hline met2 & $\begin{array}{l}\text { MetLife Building } \\
\text { (mini Sodar) }\end{array}$ & 40.75321 & -73.9764 & -- & 247 \\
\hline mgh1 & $\begin{array}{l}\text { McGraw-Hill roof, SE } \\
\text { corner }\end{array}$ & 40.75946 & -73.9812 & 17.7 & 213 \\
\hline mgh2 & $\begin{array}{l}\text { McGraw-Hill setback, } \\
\text { N side, 49th St. }\end{array}$ & 40.76011 & -73.9832 & -- & 30 \\
\hline opp & $\begin{array}{l}\text { One Penn Plaza, roof, } \\
\text { SW corner }\end{array}$ & 40.75168 & -73.9933 & 10.7 & 233 \\
\hline $\mathrm{ppz}$ & $\begin{array}{l}\text { Park Ave Plaza roof } \\
\text { station }\end{array}$ & 40.75922 & -73.9738 & -- & 182 \\
\hline \multicolumn{6}{|c|}{ Street Level Meteorological Stations } \\
\hline s1 & $\begin{array}{l}6^{\text {th }} \text { Ave btwn } 49 \text { th and } \\
50^{\text {th }} \text { St. Rockefeller } \\
\text { Center west entrance. }\end{array}$ & 40.75944 & -73.9806 & 18 & 3 \\
\hline s2 & $\begin{array}{l}\text { 49th Street btwn } 6^{\text {th }} \text { and } \\
7^{\text {th }} \text { Aves. }\end{array}$ & 40.75967 & -73.9825 & 17 & 3 \\
\hline s3 & $\begin{array}{l}12216^{\text {th }} \text { Ave., on 48th } \\
\text { St. }\end{array}$ & 40.75931 & -73.9825 & 17.6 & 3 \\
\hline s4 & $\begin{array}{l}\text { On curb in front of } 1230 \\
6^{\text {th }} \text { Ave. }\end{array}$ & 40.75879 & -73.9811 & 17.6 & 3 \\
\hline s5 & $\begin{array}{l}\text { On curb on } 6^{\text {th }} \text { btwn } \\
50^{\text {th }} \text { and } 51^{\text {st }} \text { Sts. }\end{array}$ & 40.76021 & -73.9801 & 18.9 & 3 \\
\hline
\end{tabular}




\begin{tabular}{|l|l|l|l|l|c|}
\hline s6 & $\begin{array}{l}\text { On curb, 50 } \\
6^{\text {th }} \\
\text { and Bdwy. }\end{array}$ & 40.75995 & -73.9808 & 18.2 & 3 \\
\hline s7 & $\begin{array}{l}\text { On } 7^{\text {th }} \text { Ave btwn } 48^{\text {th }} \\
\text { and } 49^{\text {th }} \text { Sts. }\end{array}$ & 40.76011 & -73.9839 & 16.1 & 3 \\
\hline s8 & $\begin{array}{l}7^{\text {th }} \text { Ave. btwn } 49^{\text {th }} \text { and } \\
50^{\text {th }} \text { Sts. }\end{array}$ & 40.7607 & -73.9834 & 16.1 & 3 \\
\hline s9 & $\begin{array}{l}50 \text { th St btwn } 6^{\text {th }} \text { and } 7^{\text {th }} \\
\text { aves. }\end{array}$ & 40.7608 & -73.9827 & 16.5 & 3 \\
\hline s10 & $\begin{array}{l}49 \text { th St btwn } 7^{\text {th }} \text { and } 6^{\text {th }} \\
\text { Aves }\end{array}$ & 40.7603 & -73.983 & 16.1 & 3 \\
\hline s11 & $\begin{array}{l}45 \text { th St btwn } 6^{\text {th }} \text { and } 7^{\text {th }} \\
\text { Aves. }\end{array}$ & 40.757 & -73.9835 & 17 & 3 \\
\hline s12 & $\begin{array}{l}54 \text { th St. btwn } 6^{\text {th }} \text { and } 7^{\text {th }} \\
\text { Aves. }\end{array}$ & 40.76278 & -73.9793 & 20 & 3 \\
\hline sit1 & $\begin{array}{l}\text { Stevens Inst. of Tech., } \\
\text { Admin Roof }\end{array}$ & 40.7608 & -73.9827 & 32 & 51.8 \\
\hline
\end{tabular}

Figure 3 shows pictures of the street level sonic anemometer installations. Maps are provided with the locations of roof top instrumentation, Figure 4, and street level instrumentation, Figure 5. 
Figure 3 shows the street level sonic anemometers at all 12 locations.
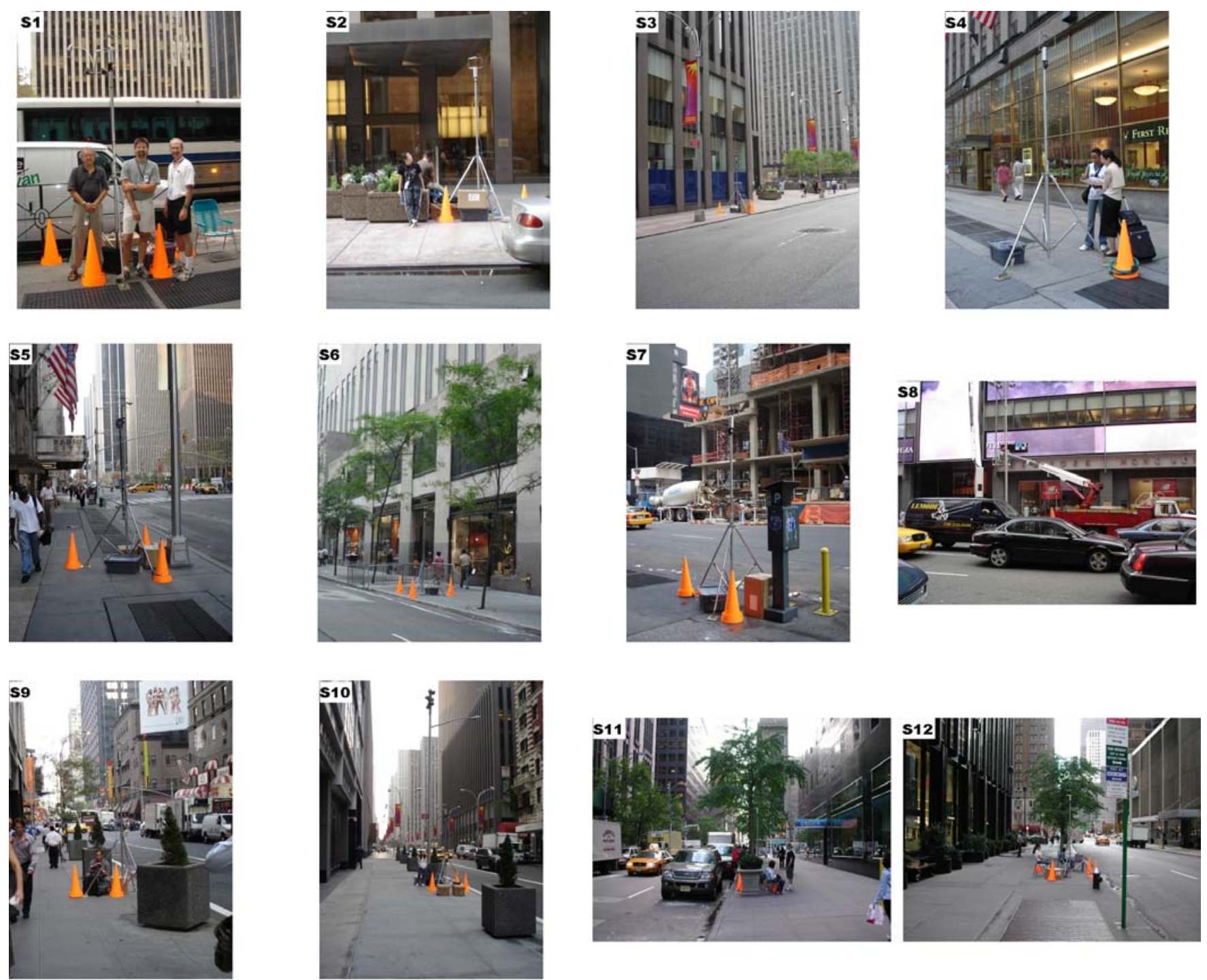

Figure 3 Photographs of the twelve street locations for sonic anemometers used in MID05 tests. 


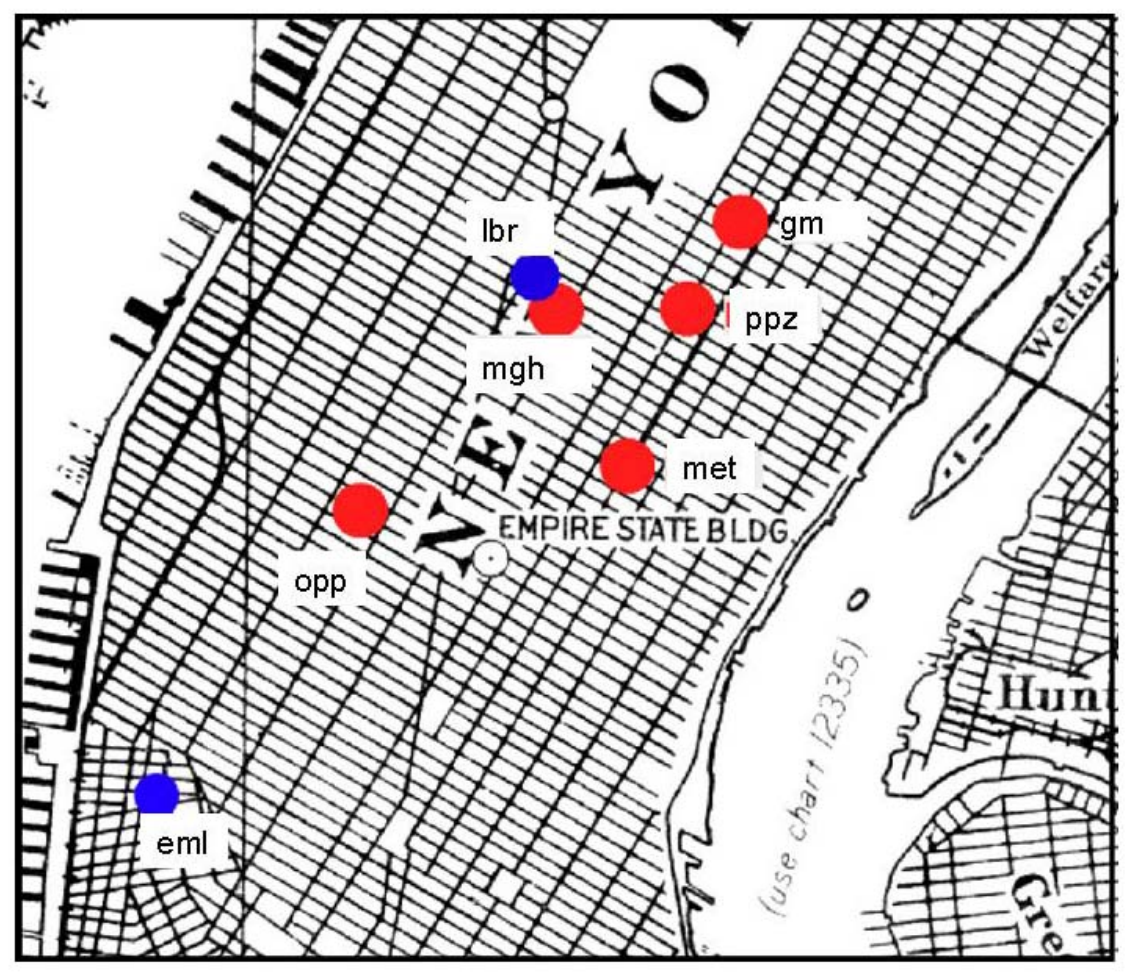

Figure 4 Map showing locations of roof top stations deployed for the MID05 experiment. 

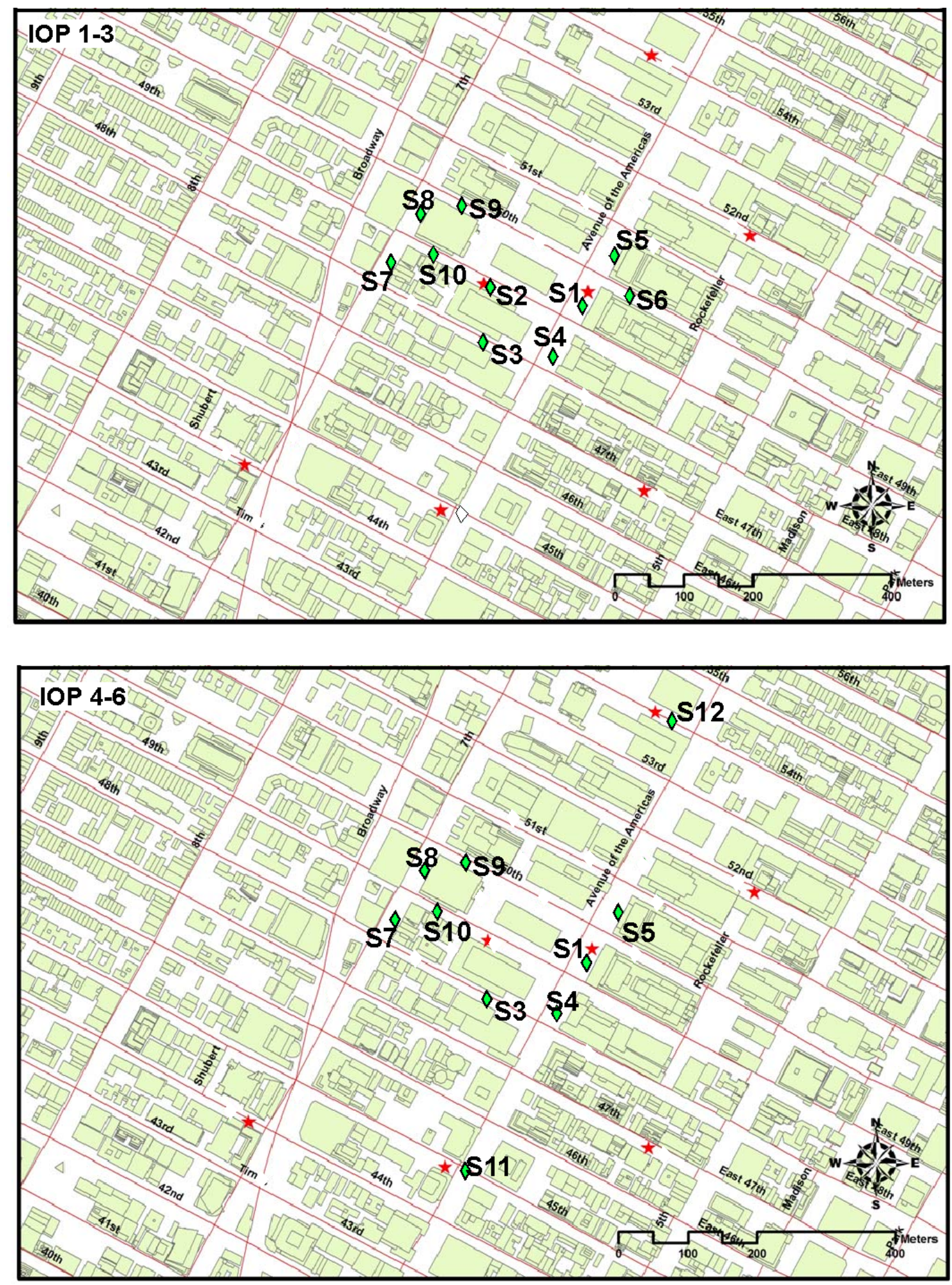

Figure 5 Map showing the locations of street sonic anemometer stations during IOPs 1 -3 and IOPs 4 - 6. 


\subsection{Deployment}

The deployments of roof top meteorological stations were all completed prior to the first IOP (Intensive Operations Period). The buildings were selected based on geographical location and ability to gain access. The installations took place within the month preceding the first IOP. Installation included mounting the equipment to the roof or setback, testing of equipment response, and calibration. Installations included two setback stations, one at the McGraw Hill building (mgh2) and one at the Lehman Brothers building (lbr2). The exact installation procedure varied due to the facilities available to us on each building. All equipment was tied into building power with a Marine battery available for backup. Each station was checked for performance one day prior to each IOP. At this time stations that were not operating were restarted.

There were six IOPs during the MID05 experiment. In order to facilitate the coordination of the large number of participants, it was arranged that the BNL staff responsible for deploying the street level sonic anemometers would meet at the Tick Tock Diner, on the northwest corner of $8^{\text {th }}$ Avenue and $35^{\text {th }}$ street, at 0400 hours (EST) each morning. Two vans loaded with the 10 3D sonic anemometers and associated towers left BNL by 0245 hours to arrive at the diner by 0400 . MID05 student team members met BNL staff and formed three teams of three people to install the equipment. The vans were driven to two staging areas, the first was 100 feet north of the northeast corner of 6th Ave and $49^{\text {th }}$ street. The second location was 300 feet east of the south east corner of $7^{\text {th }}$ Ave and $49^{\text {th }}$ street. There were 3 teams each installing 3 or 4 stations. For consistency, all BNL and student team members reported to the same location for each IOP. The installation procedure involved unloading the vans and placing the 3D Sonic anemometer and 
tower on a cart with associated equipment (computer to run operations, and installation hardware). The team members wheeled the carts and carried the equipment to each of the predesignated locations. For each of the six IOPs the same equipment and the same team members went to the same station. The towers and the anemometers were identical units. The computers collecting the data had similar physical characteristics and were running identical data acquisition software. All ten street level stations were set up and running by 0600 EST except on the first IOP where delays prevented a few of the instruments from starting on time. The sonic anemometers were aligned parallel to $6^{\text {th }}$ and $7^{\text {th }}$ Avenues or perpendicular to the street they were set up on. This insured that all the instruments would be oriented in the same direction. The compass heading for $6^{\text {th }}$ Avenue is 29 degrees. Once the stations were up and running BNL staff that deployed the equipment "roamed" from station to station confirming operation. At 1200 hours (EST) the stations were shut down, disassembled and brought back to be loaded into one of the vans waiting at the staging areas.

Between IOPs the equipment was brought back to BNL. The batteries were recharged and the anemometers were checked for proper operation. The data were copied from the laptops to thumb drives and then copied again to a central computer. Any and all repairs, if necessary, were made during this time.

Equipment failures involving street level stations were minimal. Data recovery from the street level stations was above 95 percent of the total expected. We experienced a slightly higher failure rate on the roof top stations. These failures were mainly due to exposure to extreme heat. We measured rooftop temperatures to be $50{ }^{\circ} \mathrm{C}$ and higher. The computers collecting the data were shutting down due to the excessive temperature in the enclosures. Some 
of the instrumentation power supplies overheated and failed. To compensate for the heat between the $3^{\text {rd }}$ and $4^{\text {th }}$ IOP, fans and vents were installed in the enclosures to remove the excessive heat inside and to circulate cooler air from the outside. In addition, cables were run to allow instrumentation to be located in shady areas and reduce the heat loading. These modifications corrected most of the problems with the rooftop stations. Data recovery from the rooftop stations was better than 80 percent over the six IOPs. After taking the steps to reduce heat loading on the equipment, data recovery was near $100 \%$.

After the final IOP all of the roof top equipment was removed within two weeks. Data collected from roof top equipment was similarly copied from the laptops to a central computer. All of the data was post processed and went through a QA/QC process. 


\subsection{Data Organization}

The two goals of this technical report are to provide a complete description of the meteorological measurement sub-task to the MID05 experiment, and to provide a description of the data set that accompanies this report.

\subsection{Time Recording and Averaging}

In all data in this report, all dates and times are recorded and reported in Eastern Standard Time, unless otherwise specified. The EST time zone is -5 hours different than Greenwich Mean Time. Time is written from year to seconds left to right. For example a time of 20050806,072634 is equal to 8/6/2005 7:26:34., using the United States date format, For all data sets averaged over a time-interval, the date/time mark represents the center of the averaging time. For instance, if an averaging interval is 300 seconds ( 5 minutes), the $0900 \mathrm{~h}$ average is derived from data samples from 085730 to 090230 .

\subsection{Data processing levels}

Data are segregated into three levels.

Level 0: Level 0 data are the raw data collected by the recording device available at the location. The raw $10 \mathrm{~Hz}$ data from the 3DSonic instrument are included here. Archive ASOS data downloaded from the NWS or other on line sources are collected here exactly as received. In some cases raw level 0 data are cleaned to remove obviously bad records, spikes, and dropouts. As the original data are not altered, these files remain in the level 0 
Level 1: The raw data becomes level 1 after they are processed by applying calibration coefficients, wind direction rotations, and any corrections to produce data in true physical units.

Level 2: Level 2 data are finalized, corrected, and calibrated data sets. Derived variables are also included at level 2.

In this report, Level 0 and Level 1 data are provided. A listing of all level 0 data files is presented in Appendix A and all level 1 data files are listed in Appendix B. Level 0 data are grouped into folders based on the instrumentation or location for ASOS stations. There is one Level 0 folder for each instrument used. Table 4 lists all of the Level 0 folders. These folders contain the raw data collected as part of the MID05 field study. For 3D Sonic anemometer data as received data use a file extension dat while files that have had obviously bad records removed use an extension of da1. Detailed descriptions of format and contents of the Level 0 data files are in section 5.4 .

Table 4 Station identification codes and level 0 File Folder name.

\begin{tabular}{|l|l|}
\hline \multicolumn{1}{|c|}{$\begin{array}{c}\text { Station } \\
\text { Identifier }\end{array}$} & \multicolumn{1}{c|}{ Level 0 Folder Name } \\
\hline \multicolumn{2}{|c|}{ Regional Meteorological Stations } \\
\hline cpk & cpk_Central_Park_asos \\
\hline ewr & ewr_NewarkAirport_asos \\
\hline hpn & hpn_airport_asos \\
\hline isp & isp_IslipAirp[ort_asos \\
\hline lga & lga_LaGuardiaAirport_asos \\
\hline jfk & jfK_KennedyAirport_asos \\
\hline sit1 & sit_Stevens_HoweCenter \\
\hline \multicolumn{2}{|c|}{ Roof Top Meterological Stations } \\
\hline ccny & CCNY \\
\hline
\end{tabular}




\begin{tabular}{|l|l|}
\hline eml & eml_NYC002 \\
\hline gm1 & gm1_GMBldg_roof_met \\
\hline gm2 & gm1_GMBldg_roof_Sodar \\
\hline lbr1 & lbr1_BldgA_roof \\
\hline lbr2 & lbr2_LehmanBrothers_setback \\
\hline met1 & metl1_met \\
\hline met2 & metl2_sodar \\
\hline mgh1 & mgh1_McGrawHill_roof \\
\hline mgh2 & mgh2_McGrawHill_setback \\
\hline opp & opp_OnePennPlaza_roof_met \\
\hline ppz & ppza_ParkPlaza_roof \\
\hline & street Level Meteorological Stations \\
\hline s1 & s1 \\
\hline s2 & s3 \\
\hline s3 & s4 \\
\hline s4 & s5 \\
\hline s5 & s6 \\
\hline s6 & s7 \\
\hline s7 & s8 \\
\hline s8 & s9 \\
\hline s9 & s10 \\
\hline s10 & s11 \\
\hline s11 & s12 \\
\hline s12 & \\
\hline
\end{tabular}

Level 1 data files contain data processed from the Level 0 files. Processing depends on the file but typically involves calculating averages over a time period or aligning data to correspond to true North. For example, the 3DSonic data are collected every 0.1 seconds. This data is reviewed by computer software, bad records are omitted, and a five minute (300 second) average 
of the data is calculated. Level 1 data are grouped into eight folders IOP1, IOP2, IOP3, IOP4, IOP5, IOP6, meso, and field. IOP folders contain the data from street level sonic anemometers designated as s1 - s12. This data was collected only during the 6 hour period of each IOP. The meso folder contains all of the ASOS weather station data and rooftop meteorological station data. This data were collected for the entire month of August 2005. The field folder contains the sonic anemometer data used on side-by-side comparisons of these instruments conducted at the BNL meteorological field.

\subsection{Level 1 Data File Naming Convention}

Level 1 Data files are named using a set pattern. Any file or folder names that belong to a particular IOP will begin with the IOP followed by an underscore and an optional descriptive text. For example, a folder might be named iop1_08AugustRelease.

Each data set is identified by the following three sets of ID codes:

a) Experiment ID: “mid” is the ID code for this experiment.

b) IOP ID: The entire data set is subdivided into groups called Intensive Operation periods. IOPs have a specific start and stop time as defined in the IOP information file. In addition to IOPs, an ID is available to designate meteorological data from all permanent weather stations such as NOAA ASOS stations at airports, the DCNet/URBANet stations and any BNL stations. THE ID for this data is meso. The IDs for these projects are: meso, iop1, iop2, iop3, iop4, iop5, iop6.

c) Station ID: In each IOP a set of particular stations are defined. A station here is defined as a particular set of instrumentation that produces a specific data set. For example, an ASOS airport station, a mini Sodar, and a BNL street tripod are all separate stations, even if they 
are co-located. In the case of the roof of the MetLife building, a sodar and a BNL meteorological station are co-located, so they are given stations names of met1 and $\underline{\text { met2}}$. Station IDs are provided in Table 3 above.

An example file name is mid_meso_met1_rmy3d_300av.dat. This file will contain all of the data from the met1 station (Metropolitan Life roof top, 3D Sonic anemometer), from the meso folder for the MID05 field campaign. Recall that meso data were collected for the entire month of August 2005. So this file will contain the 3D Sonic data from the Metropolitan Life rooftop on a five minute (300 second) averaging period for the entire month of August.

\subsection{Meteorological Data Summary}

\subsubsection{Sonic Anemometer Data}

The sonic anemometer wind stations provided information of the 3-dimensional turbulent winds. Stations were deployed on rooftops (eml, gm1, lbr1, met1, mgh1, opp, ppz ), building setbacks (lbr2, mgh2), and the street stations during iop1-iop6(s1-s12). The wind stations were comprised of (a) the RMY 3D sonic anemometer, (b) a tripod, 3 or $6 \mathrm{~m}$ high, or a mounting pole in the case of gm1, and (c) a data acquisition system with battery backup, AC converter, and laptop PC. The data acquisition program was a custom built program called ECCheck.exe.

Table 5 summarizes of all the 3D-sonic turbulence data collected for the UDP MID05 experiment. The roof stations and street stations are shown with their identifiers. The columns are labeled according to the date from August $2^{\text {nd }}$ to September $3^{\text {rd }}$. The first column contains the Station ID. A full description of the station locations is in Table 3. The numbers in each cell are the file sizes in Mbytes for each station and for each day. A dash means no data were collected on that day. A number of 0 indicates that less than 1 Mbyte was collected on that day. 
Table 5. Summary of data collected by each sonic anemometer station for each day of the experiment. The numbers are in Mbytes of raw data for each day. Note that a full day of $10-\mathrm{Hz}$ data produces a file of 58 Mbytes.

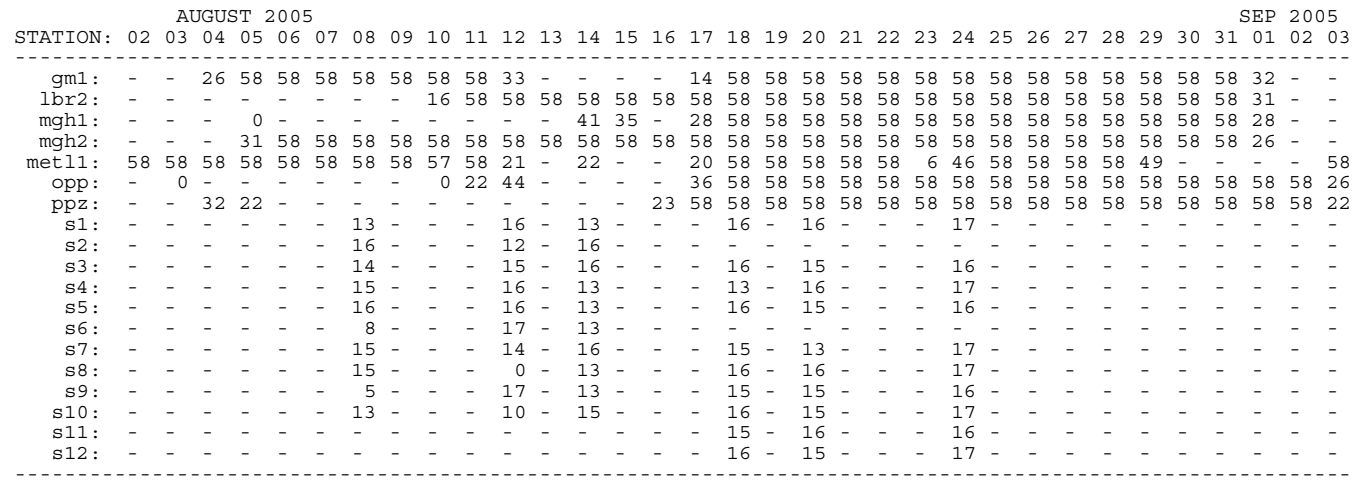

Table 5 provides the information collected from the street-level 3D sonic anemometers used during each IOP. Data were collected during each IOP from approximately 06:00 to 12:00 EST. The anemometers provided 3D velocity measurements (u, v, w), speed of sound (SOS), and sonic temperature (TSonic) every 0.1 seconds. The first tracer release of each IOP occurred 6:00. From Table 5 it is clear that data were not always collected during the IOP time period of 6:00 - 12:00. This was due to machine failures attributed to overheating. Table 6 shows the operating times of all the street level 3D sonic instrumentation based on obtaining a valid 300 second average of the $10 \mathrm{~Hz}$ data. If the Table cell is shaded gray it indicates that data were unavailable for at least 30 minutes over the time period form 6:00 - 12:00.

Table 6 Street level 3D anemometer operating times (EST).

\begin{tabular}{|c|l|l|l|l|l|l|}
\hline \multirow{2}{*}{ Station ID } & $\begin{array}{l}\text { IOP1 } \\
\text { August 8 }\end{array}$ & $\begin{array}{l}\text { IOP2 } \\
\text { August 12 }\end{array}$ & $\begin{array}{l}\text { IOP3 } \\
\text { August 14 }\end{array}$ & $\begin{array}{l}\text { IOP4 } \\
\text { August 18 }\end{array}$ & $\begin{array}{l}\text { IOP5 } \\
\text { August 20 }\end{array}$ & $\begin{array}{l}\text { IOP6 } \\
\text { August 24 }\end{array}$ \\
\hline s1 & $6: 40-12: 00$ & $5: 10-12: 00$ & $5: 00-10: 35$ & $5: 15-12: 00$ & $5: 15-12: 00$ & $5: 05-12: 00$ \\
\hline s2 & $5: 30-12: 15$ & $5: 25-6: 20$ & $5: 00-11: 55$ & -- & -- & -- \\
& & $7: 35-8: 05$ & & & & \\
& & $8: 20-9: 05$ & & & & \\
& & $10: 00-12: 05$ & & & & \\
\hline
\end{tabular}




\begin{tabular}{|c|c|c|c|c|c|c|}
\hline s3 & $6: 15-12: 00$ & $5: 45-11: 55$ & $5: 25-12: 00$ & $5: 15-12: 00$ & $5: 35-11: 55$ & $5: 20-12: 00$ \\
\hline s4 & $5: 45-12: 10$ & $5: 30-12: 00$ & $5: 25-10: 35$ & $5: 10-12: 00$ & $5: 15-12: 00$ & $5: 05-12: 00$ \\
\hline s5 & $5: 25-12: 00$ & $5: 10-12: 05$ & $5: 10-10: 30$ & $5: 30-12: 00$ & $5: 40-12: 00$ & $5: 15-12: 00$ \\
\hline s6 & $\begin{array}{c}6: 15-6: 20 \\
6: 35-8: 45 \\
9: 50-9: 55 \\
10: 05-10: 30\end{array}$ & $5: 10-12: 05$ & $5: 05-10: 30$ & -- & -- & -- \\
\hline s7 & $5: 45-12: 10$ & $5: 45-11: 45$ & $5: 25-12: 05$ & $5: 30-12: 00$ & $5: 25-12: 00^{*}$ & $5: 05-12: 00$ \\
\hline s8 & $5: 45-11: 50$ & $6: 05-12: 10$ & $\begin{array}{c}5: 05-8: 00 \\
8: 25-10: 25 \\
11: 25 \\
11: 40-11: 50 \\
12: 00-12: 05\end{array}$ & $5: 20-12: 05$ & $5: 35-12: 00$ & $5: 05-12: 05$ \\
\hline s9 & $\begin{array}{c}7: 00-7: 35 \\
7: 55-9: 25 \\
9: 50 \\
\end{array}$ & $5: 25-12: 20$ & $6: 25-11: 55$ & $5: 45-11: 55$ & $5: 30-11: 55$ & $5: 25-12: 00$ \\
\hline s10 & $\begin{array}{c}5: 30-6: 25 \\
7: 45-12: 10 \\
\end{array}$ & $5: 10-9: 30$ & $5: 15-11: 55$ & $5: 10-12: 05^{* *}$ & $5: 40-12: 00$ & $4: 45-12: 00$ \\
\hline s11 & -- & -- & -- & $5: 45-12: 00$ & $5: 20-12: 00$ & $5: 10-12: 00$ \\
\hline s12 & -- & -- & -- & $5: 55-12: 25$ & $6: 00-12: 15$ & $5: 15-12: 00 \#$ \\
\hline
\end{tabular}

* Invalid data for five-minute average at 6:20.

** Invalid data for five-minute average at 5:40, 5:55, and 6:00.

\# Invalid data for five-minute average at 10:45.

To summarize Table 6, four stations s1, s6, s9, and s10 were not successful in keeping data loss

to less than $1 / 2$ hour over the six hour sampling period in IOP 1 . This was in part due to logistical considerations which were corrected after the first IOP and in part due to equipment problems.

In IOP 2, two stations failed to collect data over the entire period, s2 and s10. In IOP3, five stations failed to collect continuous data. Four of them failed almost simultaneously at 10:30 in the morning. This was attributed to overheating of the computer equipment that is part of the data acquisition system. Fans were installed and measures were taken to provide more shade for this equipment after IOP3. Once this was done, all equipment was successful in data acquisition for the last 3 IOPs.

The rooftop and setback stations also had data acquisition problems. Nominally, the data were collected continuously for the entire month of August 2005. In Table 5, any value less than 58 
Mbytes of data collection for a day indicates partial failure. A blank in table 5 indicates that no data were collected on that day. Table 7 presents the time of data collection from rooftop sonic anemometers during the six IOPs. A shaded table cell indicates that data were not obtained for at least 30 minutes during the six hour IOP.

Table 7 Roof top and set back 3D anemometer operating times during the IOPs.

\begin{tabular}{|l|l|l|l|l|l|l|}
\hline Station ID & $\begin{array}{l}\text { IOP1 } \\
\text { August 8 }\end{array}$ & $\begin{array}{l}\text { IOP2 } \\
\text { August 12 }\end{array}$ & $\begin{array}{l}\text { IOP3 } \\
\text { August 14 }\end{array}$ & $\begin{array}{l}\text { IOP4 } \\
\text { August 18 }\end{array}$ & $\begin{array}{l}\text { IOP5 } \\
\text { August 20 }\end{array}$ & $\begin{array}{l}\text { IOP6 } \\
\text { August 24 }\end{array}$ \\
\hline gm1 & $6: 00-12: 00$ & $\begin{array}{l}6: 00-8: 25 \\
8: 40-12: 00 *\end{array}$ & -- & $6: 00-12: 00$ & $6: 00-12: 00$ & $6: 00-12: 00$ \\
\hline lbr2 & -- & $6: 00-12: 00$ & $6: 00-12: 00$ & $6: 00-12: 00$ & $6: 00-12: 00$ & $6: 00-12: 00$ \\
\hline mgh1 & -- & -- & $7: 05-12: 00$ & $6: 00-12: 00$ & $6: 00-12: 00$ & $6: 00-12: 00$ \\
\hline mgh2 & $6: 00-12: 00$ & $6: 00-12: 00$ & $6: 00-12: 00$ & $6: 00-12: 00$ & $6: 00-12: 00$ & $6: 00-12: 00$ \\
\hline metl1 & $6: 00-12: 00$ & $6: 00-8: 45$ & $6: 00-12: 00$ & $6: 00-12: 00$ & $6: 00-12: 00$ & $6: 00-12: 00$ \\
\hline opp & -- & $6: 00-12: 00$ & -- & $6: 00-12: 00$ & $6: 00-12: 00$ & $6: 00-12: 00$ \\
\hline ppz & -- & -- & -- & $6: 00-12: 00$ & $6: 00-12: 00$ & $6: 00-12: 00$ \\
\hline
\end{tabular}

* Instrument gm1 failed at $13: 50$ on August $12^{\text {th }}$ and was restarted on August $17^{\text {th }}$ at $18: 15$.

Seven roof top or setback sonic anemometers were deployed. In the first and third IOP, three of the seven were successful in collecting data. In the second IOP, four of the seven operated properly. All seven stations were available $100 \%$ of the time during the last 3 IOPs.

\subsubsection{Mini sodar data}

Mini sodar instrumentation was installed on the Met Life building on August $3^{\text {rd }} 2005$ and the General Motors Building on August $19^{\text {th }}$. After installation the sodars operated continually over the remainder of the IOPs. Therefore, data are available for all IOPs from the mini Sodar installed at the Met Life building and the $5^{\text {th }}$ and $6^{\text {th }}$ IOP for the mini Sodar installed at the General Motors building. The mini Sodar data includes a three-dimensional wind-field at 
elevations of $20-200 \mathrm{~m}$ above the instrument in $20 \mathrm{~m}$ intervals. A complete description of the raw data is in Appendix A and the description of the processed data is in Appendix B.

\subsubsection{Mesonet data}

Table 8 presents the meteorological variables measured at the mesonet stations from the nominal period starting at midnight (time 00:00:00) August 1, 2005 to September 4, 2005, time (00:00:00). Due to instrumentation problems a continuous record was not kept on all instruments as described in sections 5.4.2. The mesonet included all rooftop and setback stations, regional ASOS stations, DCUrbanet stations, CCNY stations and Stevens Institute of Technology stations. Continuous data over the time period was available from the ASOS and DCUrbanet stations.

Table 8 Meteorological variables measured at the mesonet stations.

\begin{tabular}{|c|c|c|}
\hline Station ID & $\begin{array}{l}\text { Sample } \\
\text { duration (s) }\end{array}$ & Variables measured \\
\hline cony & 1 & $\mathrm{~s}, \mathrm{~d}, \mathrm{t}, \mathrm{rh}, \mathrm{p}$ \\
\hline cpk & 1 & s,d,t,rh,ppt,p \\
\hline eml & 1 & $\mathrm{~s}, \mathrm{~d}, \mathrm{t}, \mathrm{rh}$ \\
\hline ewr & 0.5 & s,d,t,rh,p,ppt \\
\hline gm1 & 0.1 & $\mathrm{~s}, \mathrm{~d}, \mathrm{w}, \mathrm{tsonic}$ \\
\hline gm2 & 1 & $\mathrm{u}, \mathrm{v}, \mathrm{w}(20-150 \mathrm{~m})$ \\
\hline jfk & 1 & $\mathrm{~s}, \mathrm{~d}, \mathrm{t}, \mathrm{rh}, \mathrm{p}, \mathrm{ppt}$ \\
\hline lbr1 & 0.5 & $\mathrm{u}, \mathrm{v}$ \\
\hline lbr2 & 0.1 & $\mathrm{u}, \mathrm{v}, \mathrm{w}, \mathrm{tsonic}$ \\
\hline lga & 1 & s,d,t,rh,p,ppt \\
\hline met1 & 0.1 & $\mathrm{u}, \mathrm{v}, \mathrm{w}, \mathrm{ts}$ sonic \\
\hline met2 & 1 & $\mathrm{u}, \mathrm{v}, \mathrm{w}(20-150 \mathrm{~m})$ \\
\hline mgh1 & 0.1 & $\mathrm{u}, \mathrm{v}, \mathrm{w}, \mathrm{tsonic}$ \\
\hline
\end{tabular}




\begin{tabular}{|c|c|c|}
\hline mgh2 & 0.1 & u,v,w tsonic \\
\hline opp & 0.1 & u,v,w,tsonic \\
\hline ppz & 0.1 & u,v,w,tsonic \\
\hline sit1 & 1 & s,d,t,rh \\
\hline
\end{tabular}

Key to table 8.

$\begin{array}{ll}\mathrm{d}- & \text { direction }\left({ }^{\circ} \text { true North) }\right. \\ \mathrm{p}- & \text { barometric pressure }(\mathrm{mbar}) \\ \mathrm{ppt}- & \text { precipitation }(\mathrm{mm} / \mathrm{hr}) \\ \mathrm{rh}- & \text { relative humidity }(\%) \\ \mathrm{s}- & \text { wind speed }(\mathrm{m} / \mathrm{s}) \\ \mathrm{t}- & \text { temperature }\left({ }^{\circ} \mathrm{C}\right) \\ \mathrm{tsonic}- & \text { Sonic temperature }\left({ }^{\circ} \mathrm{C}\right) \\ \mathrm{u}- & \text { velocity from east }(\mathrm{m} / \mathrm{s}) \\ \mathrm{v}- & \text { velocity from north }(\mathrm{m} / \mathrm{s}) \\ \mathrm{w}- & \text { velocity from below }(\mathrm{m} / \mathrm{s})\end{array}$




\section{DATA PROCESSING}

Data were collected on the ten 3D Sonic anemometers and the two mini Sodars that were deployed during each IOP. The raw data were processed to provide 5 minute averages of the sonic anemometer data and 15 minute averages of the sodar data. Sodar data were collected from 20 - $200 \mathrm{~m}$ above the installation height at $10 \mathrm{~m}$ increments. This section describes the data processing steps and the output files that were generated.

\subsection{Sonic Anemometer Data Processing}

\section{Clean raw data files:}

Raw 3D sonic data files are described in Appendix A. Raw files are stored in the "levelo" folder

mid_level0 -> stationID_ShortDesc -> daqID\$yyyyMMdd.dat

example:

mid_level0 -> gm1_GMBldg_roof_met -> UDP01\$20050821.dat

where the stationID is described above in Table 3 (e.g. gm1), the ShortDesc is an arbitrary short descriptive string, daqID is identifies the data acquisition system, in this case the laptop assigned ID UDP01, yyyyMMdd is the data of the first record in this data file. The "dat" raw files are large, about $58 \mathrm{MB}$ per day. This file is a Level 0 file.

Each raw data file is first scanned to remove bad records. Bad records occur during rain or in rare cases $(<0.01 \%)$ during collection by the data collection program ECCheck. The program 01_clean_all_rmy3d.pl is a PERL program that reads each record of the raw data file and discards all bad records. It can be found in the directory mid -> sw -> rmy3d ->. The cleaned files are saved with names like 
mid_level0 -> gm1_GMBldg_roof_met -> gm1_yyyyMMdd.da1

where the "da1" extension identifies a cleaned raw data file. Since the only thing that has been done is to remove bad records, this is also a Level 0 data file.

\section{Average cleaned data files:}

After the raw files are cleaned of any bad records, the program 02_avg_all_rmy3d.pl produces averages of the $10 \mathrm{~Hz}$ data. This program is also in the mid -> sw -> rmy3d -> directory. Averaging times are user-defined, but typically a $300 \mathrm{sec}$ (5 min) averaging time is selected. Note, in all cases, when averages are made, the time mark of the average is the center of the averaging period. In the example above, the averaged data file is saved in the following way:

mid -> level1 -> meso -> gm1_GeneralMotors_met -> mid_meso_gm1_rmy3d_av300.dat

All processed data are stored the following hierarchy:

mid (main project data set)

level1 (first level of processed data)

meso (IOP data subdivision, includes iop1, ... iop6)

gm1_GeneralMotors_met (station ID)

mid_meso_gm1_rmy3d_av300.dat (5-min averaged data)

An example of an averaged data file is given in Table 9.

Table 9 File format for 3D sonic anemometer data processed and averaged over a 5 minute period.

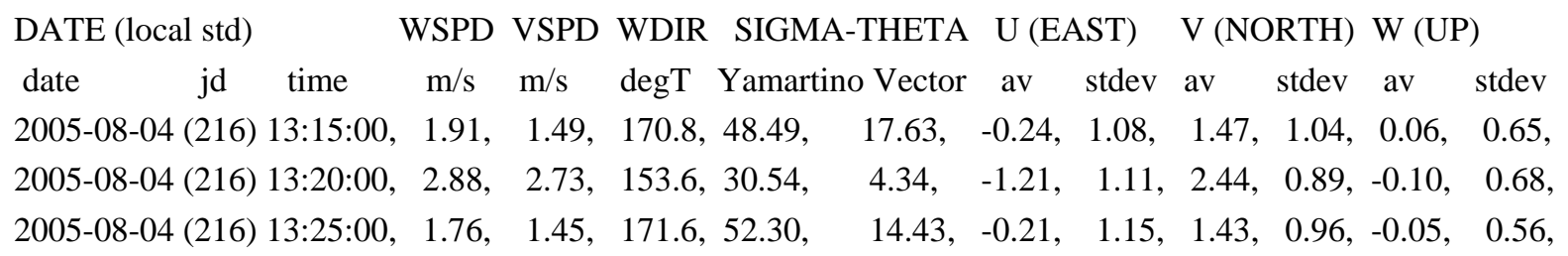


where the data columns include:

date(year -month -day),

ID (modified Julian day),

time (hours:minutes:seconds) - midpoint of the averaging period,

WSPD - scalar average wind speed $(\mathrm{m} / \mathrm{s})$

VSPD - vector average wind speed (m/s)

$\operatorname{deg} \mathrm{T}-{ }^{\circ}$ true North

Yamartino - The standard deviation of the wind direction as determined using the EPA approved Yamartino method.

Vector - The standard deviation of the wind direction as determined from the ratio of the vectormean speed and the mean scalar wind speed.

$\mathrm{U}$ (two columns) - the mean scalar average $(\mathrm{m} / \mathrm{s})$ and standard deviation of vector wind speed from the East.

$\mathrm{V}$ (two columns) - the mean scalar average $(\mathrm{m} / \mathrm{s})$ and standard deviation of vector wind speed from the East.

$\mathrm{W}$ (two columns) - the mean scalar average $(\mathrm{m} / \mathrm{s})$ and standard deviation of vector wind speed from below. 


\subsection{Mini Sodar Data Processing}

\section{$\underline{\text { Raw Sodar Processing }}$}

\begin{tabular}{|c|c|c|c|c|c|c|c|c|c|c|c|c|c|c|c|c|c|c|c|}
\hline HT & SPD & DIR & $\mathbf{W}$ & SDW & IW & GSPD & GDIR & $\mathbf{U}$ & SDU & NU & IU & SNRU & $\mathbf{V}$ & SDV & NV & IV & SNRV & NW & $\begin{array}{l}\text { SNR } \\
\mathrm{W}\end{array}$ \\
\hline 200 & 4.93 & 145 & -0.16 & 0.34 & 187 & 7.15 & 138 & -4.44 & 0.32 & 18 & 174 & 5 & 2.16 & 0.38 & 11 & 168 & 5 & 17 & 4 \\
\hline 190 & 5.25 & 136 & -0.34 & 0.31 & 201 & 6.89 & 107 & -5.02 & 0.4 & 12 & 158 & 5 & 1.53 & 0.48 & 11 & 160 & 5 & 24 & 5 \\
\hline 180 & 5.29 & 141 & -0.17 & 0.33 & 186 & 6.81 & 133 & -4.9 & 0.31 & 22 & 173 & 6 & 1.99 & 0.43 & 14 & 162 & 6 & 21 & 5 \\
\hline 170 & 5 & 151 & -0.15 & 0.3 & 186 & 6.66 & 117 & -4.26 & 0.38 & 36 & 195 & 6 & 2.61 & 0.35 & 22 & 171 & 6 & 28 & 5 \\
\hline 160 & 4.35 & 135 & 0 & 0.45 & 210 & 7.14 & 134 & -4.19 & 0.45 & 48 & 233 & 8 & 1.17 & 0.41 & 39 & 215 & 8 & 41 & 6 \\
\hline 150 & 3.98 & 141 & -0.11 & 0.39 & 270 & 9.38 & 79 & -3.71 & 0.46 & 50 & 261 & 9 & 1.46 & 0.53 & 40 & 196 & 9 & 49 & 8 \\
\hline 140 & 4.19 & 144 & -0.05 & 0.41 & 304 & 8.83 & 147 & -3.8 & 0.5 & 46 & 258 & 9 & 1.76 & 0.81 & 41 & 189 & 9 & 57 & 9 \\
\hline 130 & 3.97 & 136 & -0.06 & 0.4 & 309 & 7.38 & 99 & -3.8 & 0.57 & 52 & 290 & 10 & 1.14 & 0.79 & 37 & 193 & 10 & 50 & 9 \\
\hline 120 & 3.92 & 138 & -0.05 & 0.51 & 372 & 11.18 & 102 & -3.7 & 0.61 & 57 & 317 & 11 & 1.29 & 0.78 & 49 & 207 & 11 & 60 & 10 \\
\hline 110 & 3.9 & 140 & -0.06 & 0.51 & 389 & 7.3 & 139 & -3.63 & 0.56 & 61 & 316 & 12 & 1.42 & 0.72 & 53 & 223 & 12 & 63 & 11 \\
\hline 100 & 3.25 & 141 & -0.15 & 0.54 & 401 & 7.16 & 118 & -3.01 & 0.57 & 69 & 305 & 12 & 1.21 & 0.7 & 55 & 246 & 12 & 67 & 13 \\
\hline 90 & 3.19 & 134 & -0.06 & 0.52 & 444 & 6.45 & 101 & -3.08 & 0.58 & 67 & 320 & 15 & 0.81 & 0.6 & 64 & 278 & 15 & 66 & 15 \\
\hline 80 & 3.34 & 137 & -0.03 & 0.57 & 495 & 7.11 & 125 & -3.18 & 0.61 & 65 & 380 & 15 & 1.02 & 0.6 & 70 & 412 & 15 & 67 & 15 \\
\hline 70 & 3.13 & 133 & -0.03 & 0.6 & 508 & 8.23 & 87 & -3.04 & 0.54 & 71 & 430 & 18 & 0.76 & 0.61 & 70 & 413 & 18 & 71 & 15 \\
\hline 60 & 3.27 & 131 & 0 & 0.54 & 544 & 6.42 & 168 & -3.2 & 0.49 & 69 & 404 & 16 & 0.67 & 0.49 & 72 & 399 & 16 & 71 & 18 \\
\hline 50 & 2.39 & 119 & -0.03 & 0.44 & 565 & 4.19 & 120 & -2.39 & 0.43 & 72 & 436 & 19 & 0 & 0.31 & 72 & 618 & 19 & 72 & 18 \\
\hline 40 & 2.53 & 116 & -0.11 & 0.25 & 802 & 3.84 & 115 & -2.52 & 0.42 & 72 & 600 & 22 & -0.13 & 0.18 & 72 & 1268 & 22 & 72 & 25 \\
\hline 30 & 2.95 & 123 & -0.09 & 0.24 & 1434 & 5.79 & 121 & -2.95 & 0.49 & 72 & 778 & 21 & 0.21 & 0.05 & 72 & 4509 & 21 & 72 & 20 \\
\hline 20 & 2.55 & 95 & -0.04 & 0.09 & 4912 & 4.5 & 102 & -2.33 & 0.43 & 71 & 1159 & 16 & -1.05 & 0.15 & 72 & 4191 & 16 & 72 & 23 \\
\hline
\end{tabular}

The mini Sodar Acoustic Signal Processor (ASP) produces binary data that is stored and collected from the sodar on a regular basis. The binary records are processed by a program 
called SodarPro to produce raw ASCII data files. The ASCII data files are stored in the level0 data folder as (for example):

mid_level0 -> gm2_GMBldg_roof_sodar -> 050820dv.dat

where the previously described folder/file nomenclature is followed. The raw ASCII data file name has the yyMMdd to identify the data set. The SodarPro program divides the data into individual days.

The sodar dv files are organized in "data tables” showing all setup data and the measured winds at each level for each time. Table 10 shows an example of the data table.

Table 10 mini Sodar data table

GM1 08/20/2005 00:05:03 TO 08/20/2005 00:10:02 VR3.14 $4500 \quad 800 \quad 100 \quad 90 \quad 20 \quad 0 \quad 0$

30010155 -12001056496065 5-800800-800800-80080011029500681197000102

3 COMPONENT 19HTS ZENITH 16-16 ARA 029 SEPANG 090 MXHT 0 UNOISE 122 VNOISE 144

WNOISE 157 ANTENNA STATUS: OK AC STATUS: OFF BATTV 3.970

where the first three lines are the setup data and include the date and time period of the measurement (8-25-05 from 00:05:03 to 00:10:02) (the third line is wrapped in this example) used by the code, the fourth line is a header and each elevation level follows from the highest to lowest. A complete description of the header data is presented in Appendix A.

In the processing we convert the raw ASCII data files to raw time series files using a PERL program a01_read_sodar_dat.pl which produces time series ASCII data files that have the sodar wind data for each individual level. In this way, we can process sodar data exactly as if it were made by an individual anemometer magically held at the level. This program is found in the directory mid -> sw -> sodar ->. A time series data file is labeled

mid_level0 -> gm2_GMBldg_roof_sodar -> gm2_30.dat 
where the data file name includes the station name and the sodar level ( $30 \mathrm{~m}$ in this example). These files are used to generate the time series average files and are not presented in the data package.

Note the wspd/wdir in the sodar wind table and in the time series ascii files are computed by the sodar using the $\mathrm{u}$, and $\mathrm{v}$, wind directions and the sodar alignment angle shown in the table header. The alignment angle is set in the installation configuration file and is shown in header line 3 following the string "ARA". The time series file is a simple translation of the sodar raw ASCII wind tables. No data processing or calibrations are applied to the data in the time series files.

\subsubsection{Averaging the Sodar Time Series Data}

The time series data files are averaged with the PERL program a02_sodar_dat_avg.pl. this file is stored in the directory mid -> sw -> sodar ->

The program produces level1 data files such as:

mid -> level1 -> meso -> gm2_GMBldg_sodar -> gm2_030_av900.dat

where the sodar wind level and the averaging time are shown in the file name ( $30 \mathrm{~m}$ and $900 \mathrm{sec}$ in this example.

Program: a02_sodar_dat_avg

Version: 2.1

EditDate: $2005 / 11 / 22$

Runtime 2005-11-22 (326) 21:47:33

platform: BNL

expname: mid

iopname: meso

sitename: gm2

sensorname: 30

datapath: /Users/mike/data/urban

rawpath: /Users/mike/data/urban/mid/level0/gm2_GMBldg_roof_sodar 
rawid:_30.dat

outpath: /Users/mike/data/urban/mid/meso/gm2_GMBldg_sodar

avgsecs: 900

timezone: -5

MaxSamps: $2 \mathrm{e}+20$

Nsamp_min: 1

missing: -999

snr_threshold: 5

rotation: 29

StartTime: 2005,8,1,0,0,0

EndTime: 2005,9,30,0,0,0

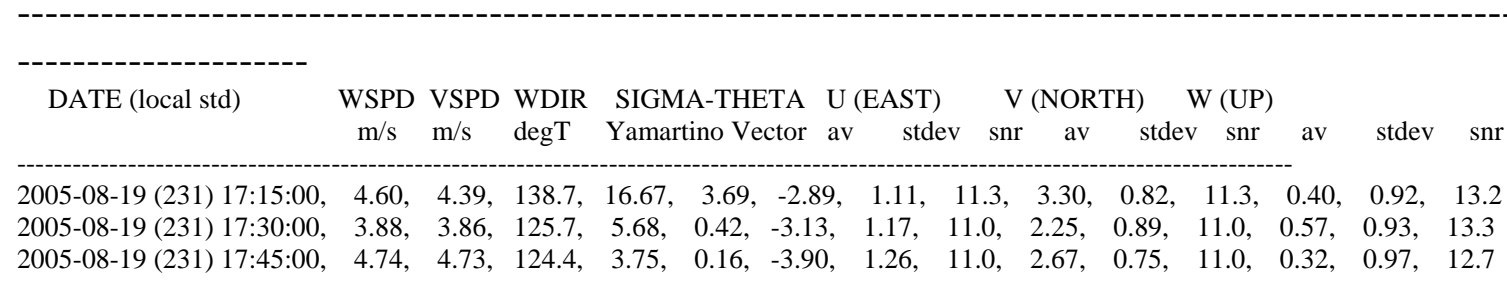

This file has an extensive header which provides information on the data processing. The time mark is the time at the center of the averaging period. WSPD is the scalar wind speed. VSPD and WDIR are vector averaged wind speed and direction based on the wind vector. In processing, the 'Av_u' and 'Av_v' columns of the raw time series files are rotated by the ARA angle described above and this vector is used to compute the average $\mathrm{U}$ and $\mathrm{V}$ columns and the vector averaged VSPD and WDIR columns. Sigma-theta by the Yamartino method uses unit vectors from the raw $\mathrm{u}$ and $\mathrm{v}$ data and sigma-theta by the vector method uses the ratio of vector speeds, VSPD to WSPD.

Techniques for removal of sporadic errors have been provided by Fleming (Fleming and Hill, 1982) and Taylor(Taylor, 1982). The Chauvenet's Criterion method was used to reject outlying points. Noise in the SODAR data occurs often as sporadic bursts which are effectively removed by the Chauvenet's criterion, described above. Occasionally an averaging time block will be primarily "bad" data and in this case Chauvenet's criterion fails to work properly because it will 
remove the good data points which are in a minority. An ad hoc method was developed from trial and error. When an averaged block is dominated by noise, the resulting value of the vector mean speed,Sv, tends to jump significantly from surrounding good data. An example time series might be $\mathrm{Sv}=\{\ldots, 1.45,1.96,2.21,22.34,15.55,1.45,1.98, \ldots\}$. The final cleaning process compares the current value of Sv with the previous value. If the time gap since the last good block is less than 2 hours and if the difference between $\operatorname{Sv}($ now)-Sv(last $)<10 \mathrm{~m} / \mathrm{s}$, then the block is recorded, otherwise it is rejected. This last procedure removes almost all of the sporadic periods of noise. The final output table contains only the data that has padded the quality assurance checks.

Table 11 Results from the three-step cleaning process for SODAR wind time series from different levels above the roof.

$\begin{array}{llll}\mathrm{Z} & \mathrm{N} & \text { Nerror } & \text { \% bad } \\ 20 & 42596 & 1105 & 3 \\ 40 & 42596 & 3817 & 9 \\ 60 & 42596 & 7767 & 18\end{array}$

The SODAR deployment period was 471.4 days and during this time the data recovery was 94.1\%. Outages were due to software crashes and a power failure. Of the recovered data, the number of bad winds, Nerror, varied from $3 \%$ at $20 \mathrm{~m}$ to $18 \%$ data loss at $60 \mathrm{~m}$. 


\section{Equipment Quality Assurance Tests}

Equipment were installed and used following manufacturers specifications. However, it is difficult to determine the quality of the data. One approach that provides confidence in data quality is comparison between outputs of instruments that are collocated. For this reason, comparisons were done between the mini Sodar and 3D Sonic anemometers on the rooftops at Met Life and the General Motors buildings.

Although the mini Sodar provides wind data at elevations of 20 - $200 \mathrm{~m}$ and the anemometer provides data at $6 \mathrm{~m}$, the general wind directions should be consistent. Wind velocities should be slightly less at the lower elevations. Figure 6 contains eight graphs of the 15 minute averaged wind data taken during the field campaign. The top row of three graphs are mini Sodar wind velocity comparisons between $20-40 \mathrm{~m}, 30-40 \mathrm{~m}$, and $40-60 \mathrm{~m}$. Beneath this are three graphs for the comparison of the wind direction at these three elevations. The bottom two graphs in the Figure are the comparison of wind speed and direction from the mini Sodar (red) at $40 \mathrm{~m}$ and the sonic anemometer (blue).

The wind speeds shown in Figure 6 at 20 and 30 meter elevations were substantially lower than at $40 \mathrm{~m}$. In addition the wind directions were much different at the lower elevations as compared to $40 \mathrm{~m}$. This suggests that the lower elevation data from the sodar are not representative of the regional winds. The comparison of sodar data at 40 and 60 meters is much better. Both the wind speed and directions are closely aligned. Considerable differences were found between the wind directions from the anemometer and the sodar located on the Met Life building, Figure 6. A quality assurance check was performed on the software used to process both data streams. The processing programs were checked on a step-by-step basis and compared with hand calculations. Both the anemometer and sodar processing programs were demonstrated to be operating 
correctly. This further supports the finding that the sodar wind data below $40 \mathrm{~m}$ are not representative of the winds in the area. After verifying the software, comparison plots of sodar and anemometer data were made for short time blocks throughout the time series.

Figure 7 is identical to Figure 6 except the data are from the mini Sodar and anemometer on the roof top of the GM building. In this case, the wind speed and direction compare as expected at all three elevations. The mini Sodar data at $40 \mathrm{~m}$ and the sonic anemometer data are consistent at this site. 


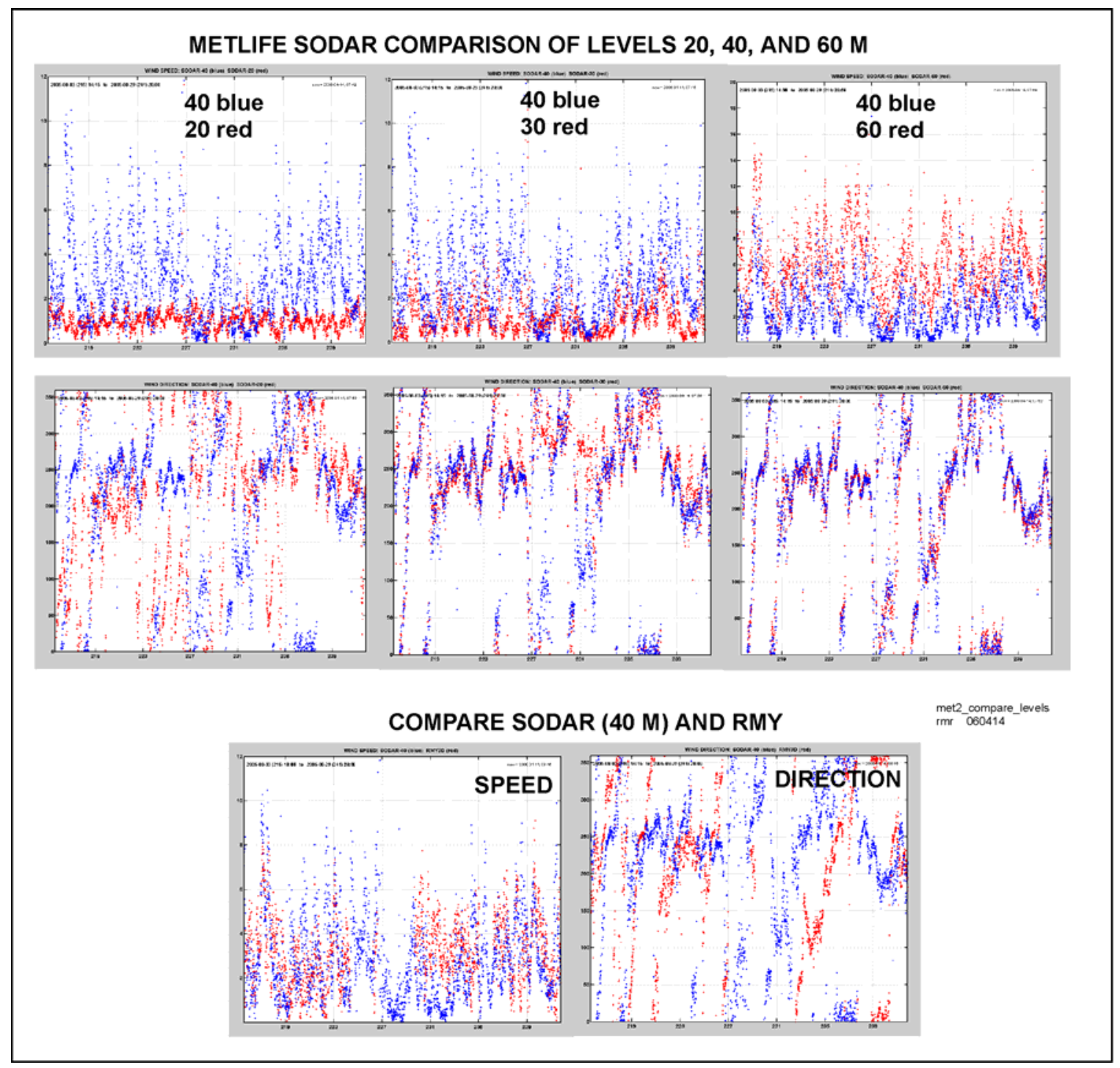

Figure 6 The mini Sodar and wind measurements from the MetLife building roof. The sodar 40-m level is used as a standard for comparison here. The 40-m measurements are shown in blue. In the top two rows the sodar wind speeds and directions at 20, 30, and $60 \mathrm{~m}$ are compared to the 40-m level. The bottom row compares the speed and direction of the sonic anemometer (red)to the sodar 40-m level (blue). All of these data are 900 sec (15 min) averages. 


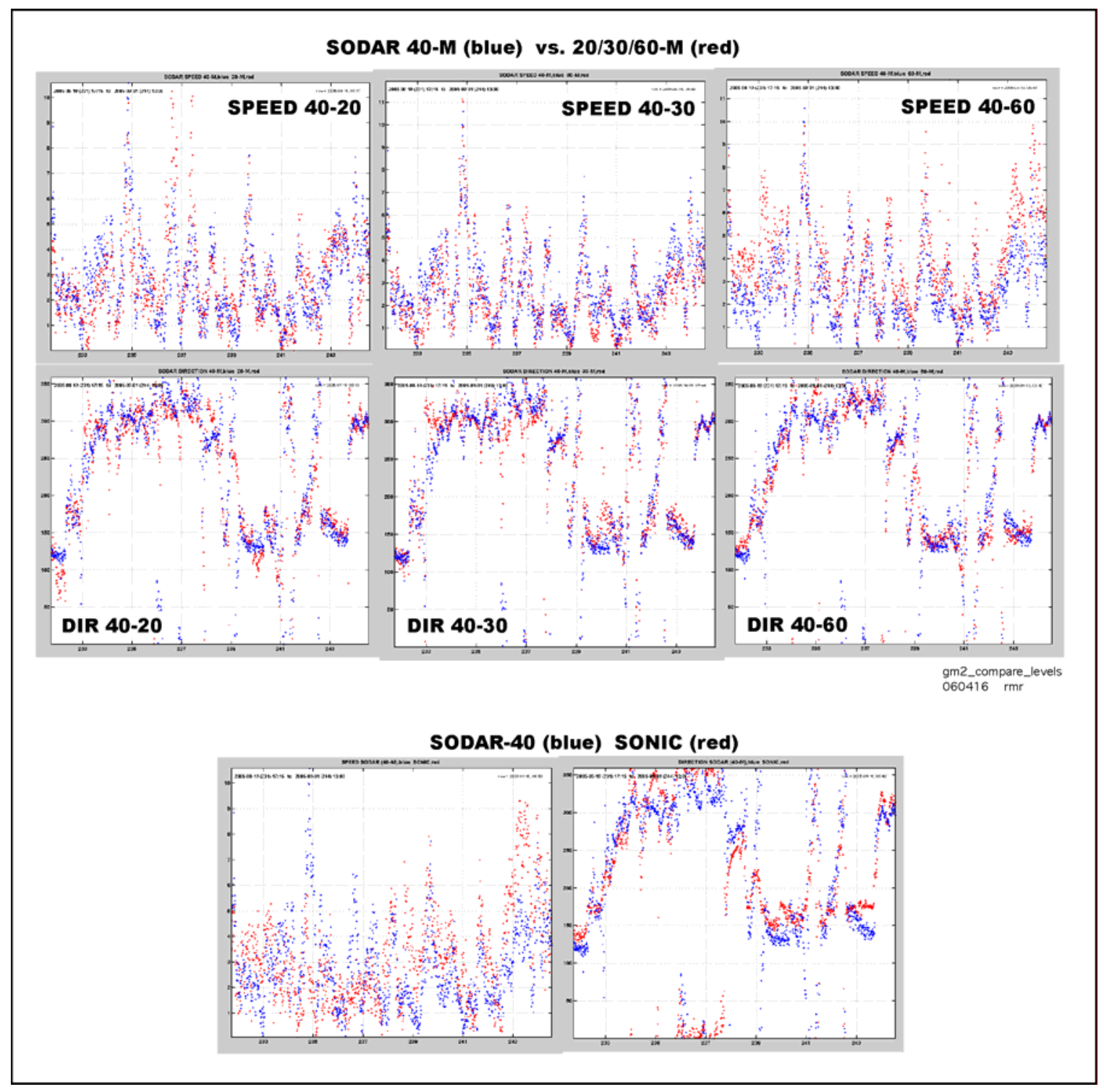

Figure 7 The mini Sodar and wind measurements from the General Motors Building (gm) roof. The sodar 40-m level is used as a standard for comparison here. The 40-m measurements are shown in blue. In the top two rows the sodar wind speeds and directions at 20, 30, and $60 \mathrm{~m}$ are compared to the 40-m level. The bottom row compares the speed and direction of the sonic anemometer to the sodar 40-m level. All of these data are $900 \mathrm{sec}(15 \mathrm{~min})$ averages.

\subsection{Windrose Intercomparison at Mesonet Stations}

Figure 8 is a compilation of simple wind roses for stations in the mesonet array during the MID05 test period in the month of August, 2005 for 16 different direction bins. Wind roses are presented for the ASOS stations at Kennedy Airport (JFK) and LaGuardia airport (LGA); roof top 3Dsonic anemometers at One Penn Plaza (OPP), Metropolitan Life Building (MET1), Park 
Plaza (PPZ), General Motors Building (GM1) and the McGraw Hill building (MGH1); and mini Sodars at $60 \mathrm{~m}$ above the roof top at Metropolitan Life (MET2) and General Motors (GM2) buildings. The wind rose shows the percentage of time that the wind is blowing from one of the 16 direction bins. Wind speeds are color coded with the inner red region representing $0-3 \mathrm{~m} / \mathrm{s}$, the white region is $3-7 \mathrm{~m} / \mathrm{s}$, and the outer red regions is $>7 \mathrm{~m} / \mathrm{s}$. In some cases the percentages do not add up to $100 \%$ because of equipment failures that occurred during the month (heat problems). Nevertheless, one can see a tremendous scatter in the wind directions at the different stations. This highlights the need for very localized data to accurately predict dispersion in urban environments..

The ASOS stations at JFK and LGA airports are consistent and show a dominant southerly wind. The LGA station also shows a sea/land breeze related to flow in and out of Long Island Sound. Examining the figure it is clear that the wind patterns at these two airport ASOS stations are considerably different than the Manhattan Mesonet stations.

The raw data sets were carefully checked to assure that the instruments were installed correctly and that the data processing was done properly. The exact same processing software was used in the BNL meteorological field comparisons (section 7.2) and the results there were quite good (average wind speeds within $0.1 \mathrm{~m} / \mathrm{s}$ and wind direction within $2^{\circ}$ ).

The miniSodar data shown in Figure 8 are from time series of winds at the $60 \mathrm{~m}$ range gate. Comparison with the wind roses at 40,50 , and $70 \mathrm{~m}$ show similar and consistent results. The comparison between MET1 (MetLife building 6-m tower) and MET2 (MetLife building miniSodar at $60 \mathrm{~m}$ ) is somewhat similar. However, they are dissimilar enough as to call into question the value of roof stations as a predictor of higher level winds. The comparisons of 
GM1 (General Motors building rooftop) and GM2 (General Motors building sodar at $60 \mathrm{~m}$ ) are different as discussed above.

The 3D sonic rooftop wind direction measurements from OPP, PPZ and GM1, are generally similar showing, roughly, a N-S preference and a dominant southerly flow.

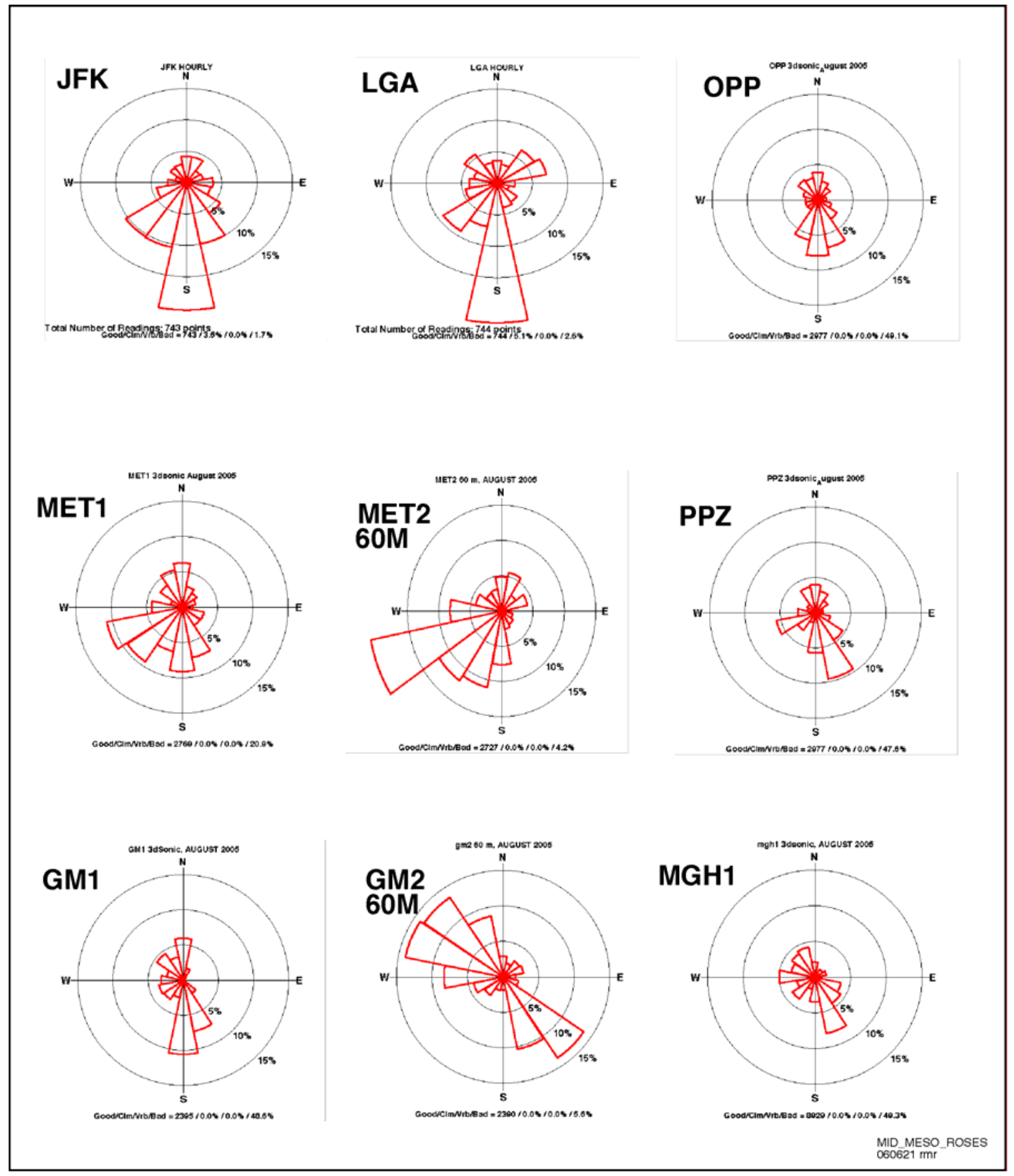

Figure 8 Directional Wind Rose from nine mesonet stations in August 2005.

42 of 88 


\subsection{Wind Intercomparisons in the BNL Meteorological Field}

\subsubsection{Field Comparisons of the mini Sodar at BNL}

A mini Sodar has been evaluated through comparison of measurements made at the BNL

meteorological tower. The BNL meteorological tower has calibrated anemometers at heights of $10 \mathrm{~m}$ and $88 \mathrm{~m}$. The sodar was placed in the meteorological field approximately $100 \mathrm{~m}$ from the base of the tower. A comparison of the winds is shown in the figures below:

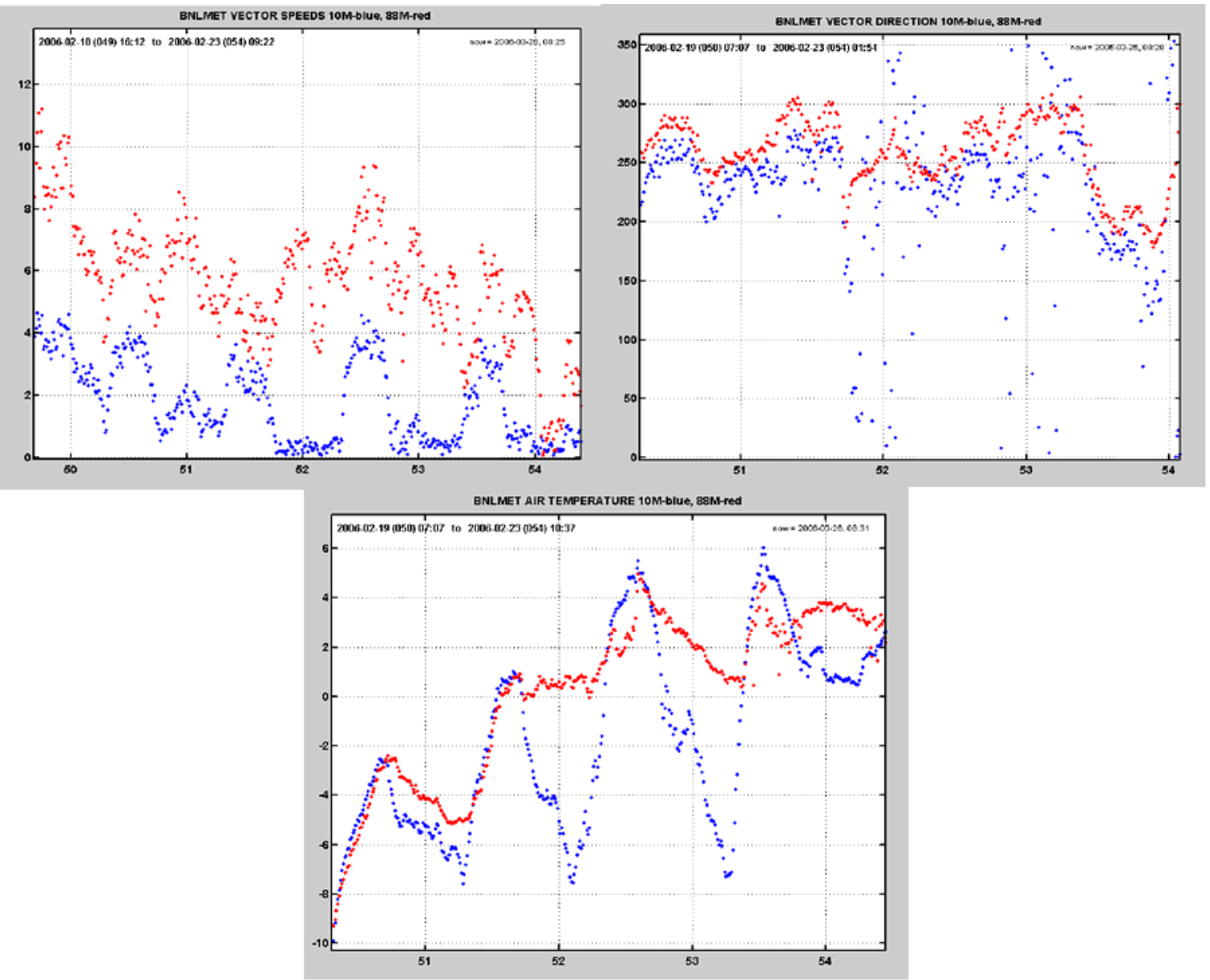

Figure 9 The vector speed, vector direction, and air temperature at $10 \mathrm{~m}$ (blue) and $88 \mathrm{~m}$ (red) from the BNL meteorological tower. 


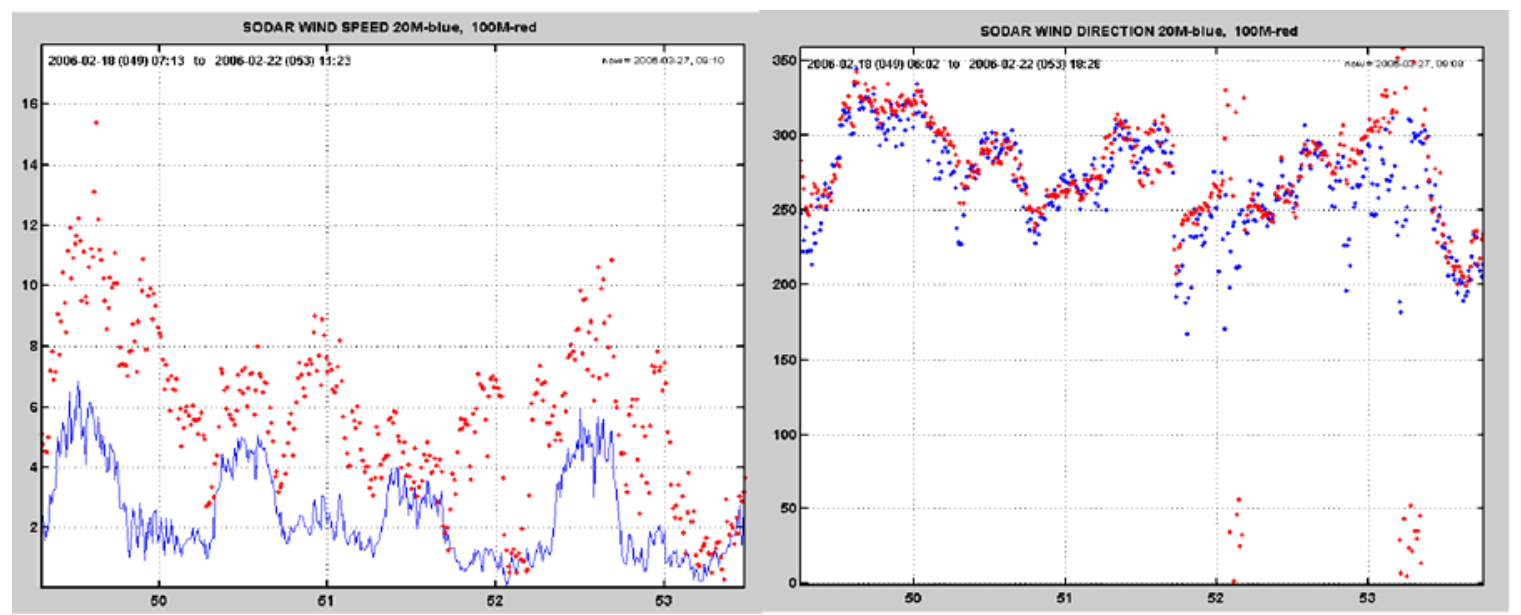

Figure 10 Wind speed and direction measured by the mini Sodar at $20 \mathrm{~m}$ (blue) and $100 \mathrm{~m}$ (red).

Prior to field deployment the new ASC miniSodar (called SODAR-2) was operated in the BNL meteorological field (BNLMET) from June 21 - June 30, 2005 where the 90 m tower collects precise wind measurements at $10 \mathrm{~m}$ and $88 \mathrm{~m}$. The sodar averaged wind profiles for two minutes. BNLMET data are sampled at one hertz and the data acquisition system makes one minute averages.

The sodar data were processed with the same processing routines as used for the Manhattan study on the General Motors building (gm2). Nine hundred second averages, 15 minutes, were calculated of the wind speed and direction at $10 \mathrm{~m}$ intervals from $20 \mathrm{~m}$ to $200 \mathrm{~m}$. Figure 11 compares data from three levels, (20 m, $40 \mathrm{~m}$, and $90 \mathrm{~m}$ ) with the tower wind measurements taken from the $88 \mathrm{~m}$ level. The wind roses consider three groups, $0-3 \mathrm{~m} / \mathrm{s}$ (red near center of the figure), $3-7 \mathrm{~m} / \mathrm{s}$ (white regions), and $>7 \mathrm{~m} / \mathrm{s}$ red in the outer region of the circle. The figure demonstrates that the comparison between the $90 \mathrm{~m}$ sodar and the $88 \mathrm{~m}$ tower wind roses 
is quite good. The wind roses from lower levels show the expected effects of friction and summer time nocturnal inversions. The sodar data file header below shows the processing parameters.

\section{SODAR2 DATA AVERAGING HEADER}

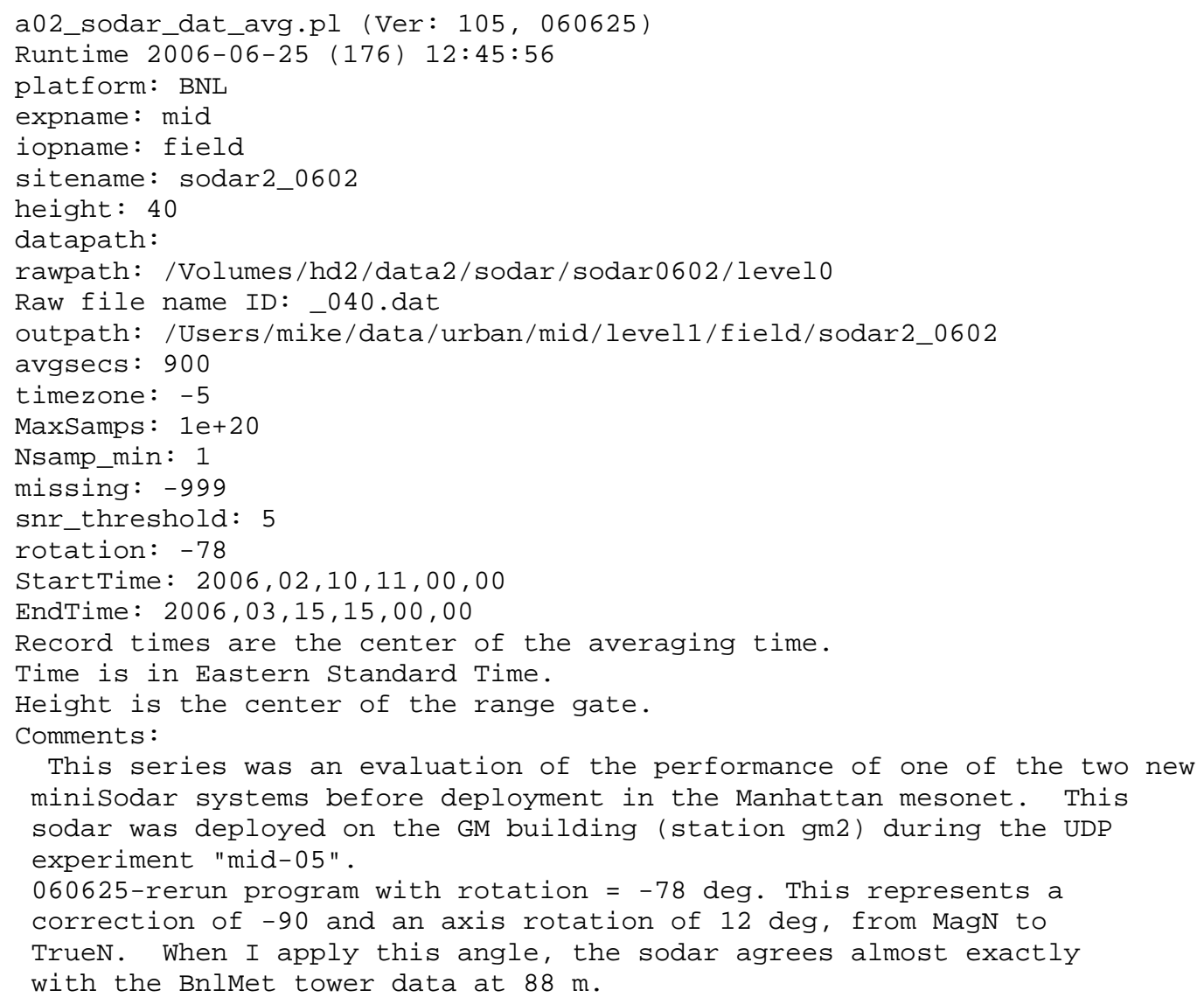




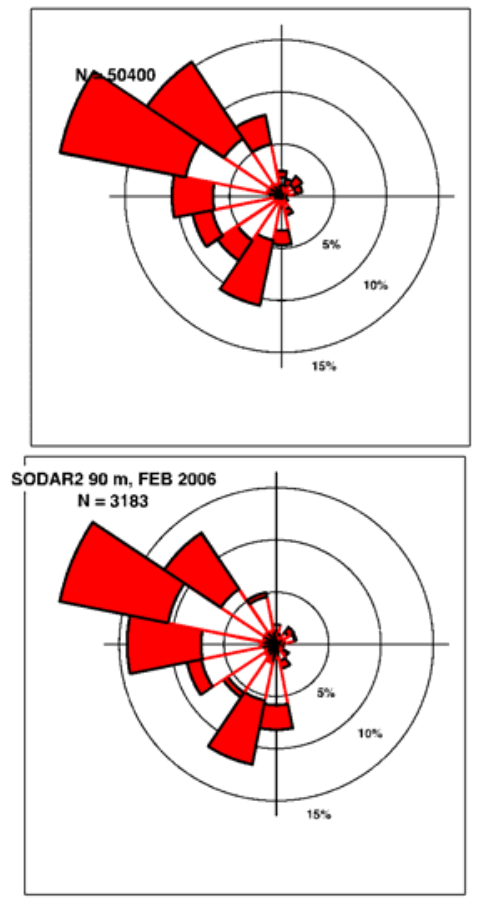

BNLMET 88M

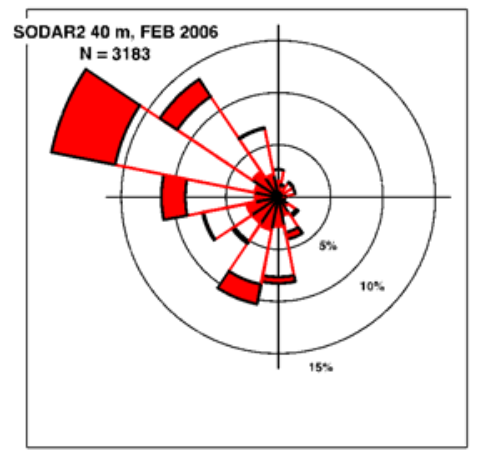

SODAR2 $90 \mathrm{M}$

SODAR2 $40 \mathrm{M}$

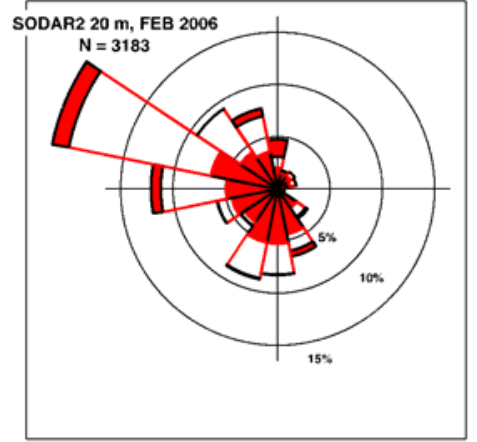

SODAR2 $20 \mathrm{M}$

Figure 11 Comparison of Wind Speed and Direction for the miniSodar at 20, 40, 60, and 90 meters and the BNL meteorological tower at $88 \mathrm{~m}$.

\subsubsection{Side-by-side comparison of 3D Sonic Anemometers}


After the field campaign in August 2005, the 3D Sonic anemometers were brought back to BNL. In September, these instruments were tested at the BNL meteorological field in a side-by- side comparisons over several days. Four series of tests were completed based on when the instrumentation was returned. In general, one or more instruments were used in more than one series to provide a measure of consistency between tests. Figure 12 plots the mean speed and mean direction of each group. This was a mid-summer exercise and the winds were generally weak and showed strong nocturnal variability. Average wind speeds for the days tested ranged from $0.3 \mathrm{~m} / \mathrm{s}$ to $1.4 \mathrm{~m} / \mathrm{s}$. Table 12 compares the deviation from the group mean speed and direction based on 5 minute averages. The horizontal speed and direction are compared in this analysis. In almost all cases, each anemometer agreed with the group mean by, $<0.1 \mathrm{~m} / \mathrm{s}$ in speed and by $<2$ deg in direction. Note the direction bias is dependent on proper alignment during installation. A few instruments showed high standard deviations about the mean in wind direction. 


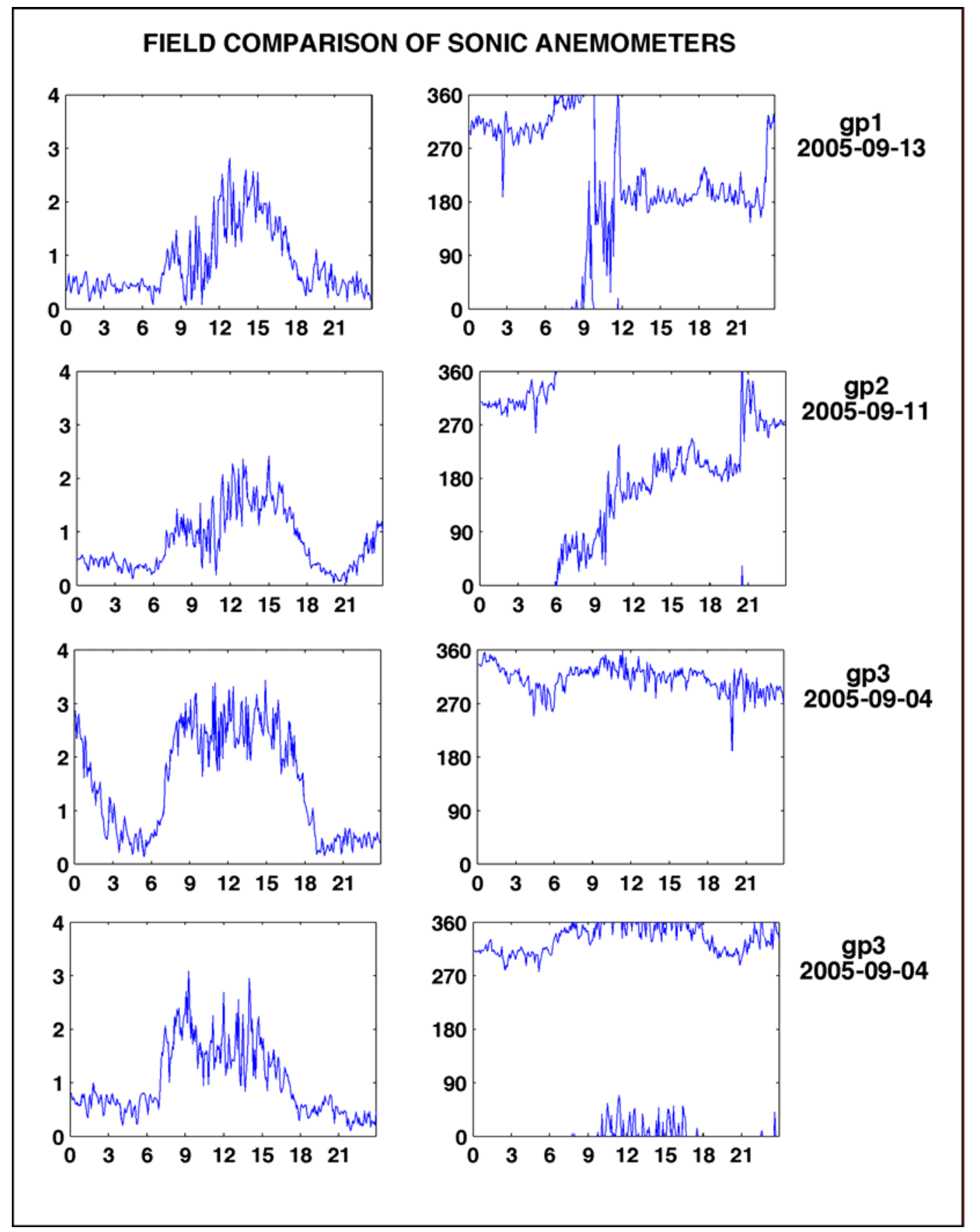

Figure 12 The group mean wind speed (left column) and direction (right column) during each of the intercomparison periods. 
Table 12 The mean bias and standard deviation for each sonic anemometer during the four-part field test.

GROUP: GP1 MEAN VEL: $0.4 \mathrm{~m} / \mathrm{s}, 204.1 \mathrm{deg}$

START: 20050913, 000000 , STOP: 20050913, 235500, Npts $=288$

\begin{tabular}{lllll} 
& \multicolumn{2}{c}{ Wind Speed } & \multicolumn{1}{c}{ Wind Direct } \\
RMY SN & BIAS & STD DEV & BIAS & \multicolumn{1}{c}{ STD DE } \\
SN 320 & 0.039 & 0.100 & -0.1 & 15.7 \\
SN 358 & 0.039 & 0.118 & -0.1 & 14.4 \\
SN 362 & 0.061 & 0.110 & 1.0 & 15.5 \\
SN 363 & 0.055 & 0.108 & 1.1 & 14.5 \\
SN 364 & -0.027 & 0.230 & 0.7 & 24.6 \\
SN 365 & 0.064 & 0.107 & 0.3 & 14.0 \\
SN 366 & 0.056 & 0.253 & -1.1 & 26.8 \\
SN 367 & 0.062 & 0.110 & -1.7 & 16.5 \\
SN 597 & 0.017 & 0.094 & -1.4 & 14.7 \\
SN 607 & 0.017 & 0.512 & 5.1 & 53.0
\end{tabular}

GROUP: GP2 MEAN VEL: $0.3 \mathrm{~m} / \mathrm{s}, 194.3 \mathrm{deg} T$

START: 20050911,000000, STOP: 20050911,235500, Npts $=288$

\section{Wind Speed}

RMY SN

SN 595

SN 602

SN 608

\section{BIAS}

๑. 066

STD DEV

0.055

0.087

0.235

0.241

0.341
Wind Direction

\section{BIAS STD DEV}

$-3.0 \quad 23.8$

$\begin{array}{ll}-2.8 & 23.9\end{array}$

$7.0 \quad 35.9$

GROUP: GP3 MEAN VEL: $1.4 \mathrm{~m} / \mathrm{s}, 320.4$ degT

START: 20050901, 000000 , STOP: 20050901, 235500, Npts $=288$

\begin{tabular}{|c|c|c|c|c|}
\hline & \multicolumn{2}{|c|}{ Wind Speed } & \multicolumn{2}{|c|}{ Wind Directi } \\
\hline RMY & BIAS & STD DEV & BIAS & STD \\
\hline SN 358 & 0.055 & 0.108 & -1.0 & 5.5 \\
\hline SN 601 & -0.046 & 0.521 & -2.5 & 23.4 \\
\hline 606 & 0.004 & 0.139 & -0.5 & 7.4 \\
\hline 610 & ๑. . 010 & 0.106 & -0.1 & 5. \\
\hline SN 611 & 0.003 & 0.100 & 0.8 & 5. \\
\hline 614 & 0.032 & 0.110 & -0.5 & 5. \\
\hline N 621 & 0.046 & 0.108 & -1.1 & 5. \\
\hline
\end{tabular}

GROUP: GP4 MEAN VEL: $0.9 \mathrm{~m} / \mathrm{s}, 343.3 \mathrm{degT}$

START: 20050904, 000000, STOP: 20050904, 235500, Npts $=288$

\section{Wind Speed}

RMY SN

SN 320

SN 358

SN 364

SN 598

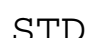

BIAS

0.009

$\odot .026$

0.094

$-0.023$
0.109

0.124

0.178

0.104

\section{Wind Direction}

BIAS

$-0.4$

$-0.3$

$-0.0$

$-1.0$
STD DEV

7.0

8.0

8.3

7.5 


\begin{tabular}{|c|c|c|}
\hline 608 & 0.014 & 0.438 \\
\hline SN 616 & 0.013 & 0.110 \\
\hline
\end{tabular}

Table 12 shows that some of the 3D sonic anemometers exhibit a large standard deviation in the wind direction. For example, machines SN 607 in group 1, SN 608 in group 2 and 4. Further examination of the performance of these machines is presented in Figure 13 which contains a comparison of the difference between the mean wind direction of the group and the wind direction of three individual instruments, SN 320, SN 364, and SN 607. From table 12, the mean direction $204^{\circ}$ true North and instrument SN 607 had a bias of 5.1 and standard deviation of $53^{\circ}$. This is considerably more scatter than the others and this is evident in Figure 13. This suggests that values for wind speed and direction can be viewed with high confidence for instruments SN 320 and SN 364, while this is not the case for instrument SN 607. Based on the data in Figure 12, the performance of instruments SN 601 and SN 608 are also suspect. 

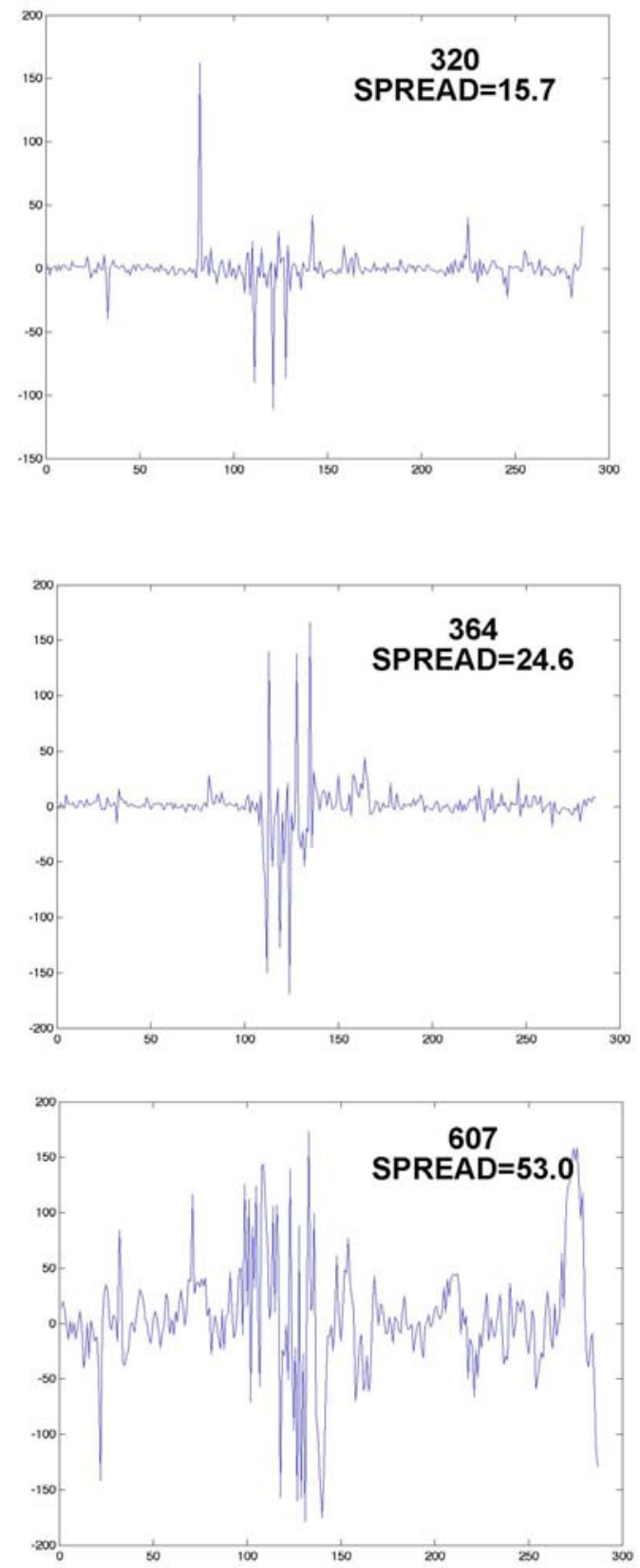

Figure 13 Comparison of measured wind direction to the group mean wind direction for instruments SN 320, SN 364, and SN 607 in group 1. 


\section{Appendix A: LEVEL 0, TOP LEVEL FOLDERS}

Each instrument used in the MID05 has a level 0 folder containing all of the raw data collected during the field campaign. A listing of all of the folders in the level 0 folder is shown below.

\begin{tabular}{|c|c|c|c|c|c|c|c|}
\hline \multirow[b]{2}{*}{4} & \multirow[b]{2}{*}{$r m r$} & \multirow[b]{2}{*}{$r m r$} & \multicolumn{2}{|c|}{ Date } & \multicolumn{3}{|c|}{ Folder Name } \\
\hline & & & 136 & Oct & 27 & 2005 & cony \\
\hline 4 & $r m r$ & $r m r$ & 136 & Oct & 27 & 2005 & cpk_Central_Park_asos \\
\hline 3 & $r m r$ & $r m r$ & 102 & Oct & 27 & 2005 & eml_nyc००2 \\
\hline 4 & $r m r$ & $r m r$ & 136 & Oct & 27 & 2005 & ewr_NewarkAirport_asos \\
\hline 54 & $r m r$ & $r m r$ & 1836 & Oct & 27 & 2005 & gm1_GMBldg_roof_met \\
\hline 16 & $r m r$ & $r m r$ & 544 & Oct & 27 & 2005 & gm2_GMBldg_roof_sodar \\
\hline 5 & $r m r$ & $r m r$ & 170 & Oct & 27 & 2005 & hpn_airport_asos \\
\hline 4 & $r m r$ & $r m r$ & 136 & Oct & 27 & 2005 & isp_IslipAirp[ort_asos \\
\hline 3 & $r m r$ & $r m r$ & 102 & Oct & 27 & 2005 & jfk_KennedyAirport_asos \\
\hline 4 & $r m r$ & $r m r$ & 136 & Oct & 27 & 2005 & lbr1_BldgA_roof \\
\hline 49 & $r m r$ & $r m r$ & 1666 & Oct & 27 & 2005 & Ibr2_LehmanBros_setback \\
\hline 3 & $r m r$ & $r m r$ & 102 & Oct & 27 & 2005 & lga_laguardia airport_asos \\
\hline 63 & $r m r$ & $r m r$ & 2142 & Oct & 27 & 2005 & metl1_met \\
\hline 33 & $r m r$ & $r m r$ & 1122 & Oct & 27 & 2005 & met12_sodar \\
\hline 41 & $r m r$ & $r m r$ & 1394 & Oct & 27 & 2005 & mgh1_McGrawHill_roof \\
\hline 59 & $r m r$ & $r m r$ & 2006 & Oct & 27 & 2005 & mgh2_McGrawHill_setback \\
\hline 60 & $r m r$ & $r m r$ & 2040 & Oct & 27 & 2005 & opp_onePennPlaza_roof_met \\
\hline 84 & $r m r$ & $r m r$ & 2856 & Oct & 27 & 2005 & ppza_ParkPlaza_roof \\
\hline 16 & $r m r$ & $r m r$ & 544 & Oct & 27 & 2005 & s1 \\
\hline 16 & $r m r$ & $r m r$ & 544 & Oct & 27 & 2005 & s10 \\
\hline 10 & $r m r$ & $r m r$ & 340 & Oct & 27 & 2005 & s11 \\
\hline 10 & $r m r$ & $r m r$ & 340 & Oct & 27 & 2005 & s12 \\
\hline 10 & $r m r$ & $r m r$ & 340 & Oct & 27 & 2005 & s2 \\
\hline 17 & $r m r$ & $r m r$ & 578 & Oct & 27 & 2005 & s3 \\
\hline 17 & $r m r$ & $r m r$ & 578 & Oct & 27 & 2005 & s4 \\
\hline 17 & $r m r$ & $r m r$ & 578 & Oct & 27 & 2005 & s5 \\
\hline 12 & $r m r$ & $r m r$ & 408 & Oct & 27 & 2005 & s6 \\
\hline 16 & $r m r$ & $r m r$ & 544 & Oct & 27 & 2005 & s7 \\
\hline 19 & $r m r$ & $r m r$ & 646 & Oct & 27 & 2005 & s8 \\
\hline 16 & $r m r$ & $r m r$ & 544 & Oct & 27 & 2005 & s9 \\
\hline 4 & $r m r$ & $r m r$ & 136 & Oct & 27 & 2005 & sit_Stevens_HoweCenter \\
\hline
\end{tabular}

The level 0 folder contains all of the raw data that are collected from the following sources:

ASOS: (cpk, ewr, hpn, isp, jfk, lga): NOAA automated weather stations. The raw file here has a name such as cpk2005 which has data from 1 Jan 2005 to 1 Sep 2005 which covers both the MSG and MID experiments. The header of the ASOS file is shown below

Time correction: $\odot$ 


\begin{tabular}{|c|c|c|c|c|c|c|c|c|c|}
\hline Date & JD & Time & & wspd (m/s) & $\begin{array}{l}\text { wdir } \\
\text { (degt) }\end{array}$ & $\operatorname{tair}(\mathrm{C})$ & $\operatorname{tdew}(\mathrm{C})$ & $r h(\%) \operatorname{slp}(\mathrm{hPa})$ & $\operatorname{ppt}(\mathrm{mm})$ \\
\hline $2005-01-01$ & $(001)$ & $\odot \odot: 51: 00$ & 5.7, & 230 & 11.1 & 5.6 & 69 & $1016.8, \quad 0.0$ & \\
\hline $2005-01-01$ & $(001)$ & $01: 51: 00$ & 3.6 , & 240 & 11.1, & 5.6 & 69 , & 1016.8, & \\
\hline $2 \odot \odot 5-01-\odot 1$ & $(\odot \odot 1)$ & $\odot 2: 51: \odot \odot$, & 4.1, & 240 & 11.1 & 5.6, & 69 , & 1016.8, & \\
\hline $2005-01-01$ & $(001)$ & $03: 51: 00$ & 4.1, & 240 & 10.6 & 5.6 & 71 & 1016.8, & \\
\hline
\end{tabular}

ASOS records are reported hourly, typically at 50 minutes after the hour, and are a five-minute average of the measurement suite. The file contains columns for the date, Julian date, time (EST), wind speed $(\mathrm{m} / \mathrm{s})$, wind direction (True North), air temperature $\left({ }^{\circ} \mathrm{C}\right)$, dew point temperature $\left({ }^{\circ} \mathrm{C}\right)$, barometric pressure $(\mathrm{kPa})$, and precipitation $(\mathrm{mm})$.

BNL Rooftop and Setback Sonics (gm1, lbr2, metl1, mgh1, mgh2, opp, ppza): BNL installed seven sonic anemometer stations on roof tops and setbacks. These are all identical instruments collecting three components of wind and the sonic temperature at a $10 \mathrm{~Hz}$ sampling rate.

There are two file types in this folder. The original files have names such as UDP01\$20050805.dat where the first five characters designate the data acquisition computer and the last eight are the starting date for the file.

The second type of file has a form such as gm1_20050826.da1 where the location is identified along with the date. These files called the "da1" files are exactly the same as the first type but the data here are cleaned and any short records are removed. The header and a few data lines for the da1 files is given below:

PROGRAM rmy3d_clean_v3b.pl (ver v3b, 0509030): requires two arguments. The program reads each raw data file and combs through for short data lines. Experience indicates that at 
worst, we get about $0.3 \%$ bad data lines. When short lines occur several other lines are lost completely. Spike values are also bad lines with missing data. All short or bad lines (spikes) are removed from the file.

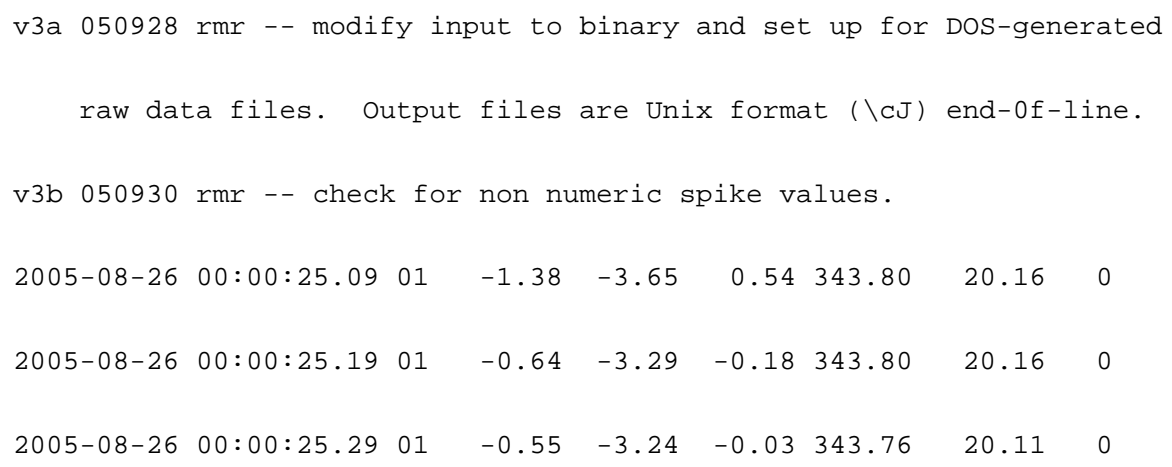

BNL Rooftop Sodar Stations (gm2, metl2): Two mini Sodar systems were deployed on two different rooftop locations in Midtown. The raw data from the sodar system is organized in daily files with names such as $050807 d v$.dat which gives the date as yyMMdd as the file name.

The data in the raw files are organized into data tables. A new data table is created at the end of each averaging period (1 minute) for this experiment. A typical table is shown here (pardon the small print). Header information follows. 


\begin{tabular}{|c|c|c|c|c|c|c|c|c|c|c|c|c|c|c|c|c|c|c|c|c|c|c|}
\hline 6rest & 93 & 15ked & is be:t & 1:10 $\mathrm{rg}$ & $03 y$ & 15rabe & 9:F & :a1 Wh & 25 & de & in & 190 & 57 & 10 & $t$ & 9 & मант & Ba & IT: & $1 \%$ & FABTLIT & Majte \\
\hline 120 in & $15 \mathrm{~s}$ & -1200 & 105 & 445 & E 3 & $3-60$ & $10-6$ & 6 and & 4004 & 0110 & i1t & 54 & de 1 is & 700 & 14 & 2 & & & & & & \\
\hline $3 \mathrm{corT}$ & news & Ltats: & 2ЕнТTH & $16-14$ & min & its sEF & $a z$ & 6. мпнт & 4 & wot be & & 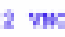 & $\operatorname{tg}$ & 12 кus & & 42 & คВтЕหM & str & TUE: & WA & SThTUS: & $\mathrm{Wh}$ \\
\hline Hт & STD & Dth & W & Bts & I & "ars & का & v & sot & หบบ & IU & BMn & $y$ & Brrt & $\mathrm{Nr}$ & $\boldsymbol{I V}$ & sнат & $\mathrm{my}$ & ตнม & & & \\
\hline 200 & 9.93 & 5413 & -9.5 & 9.23 & 3 & 44.45 & 434) & -9.14 & dit $3 t$ & $y$ & 21 & 4 & 43.5 & 45.47 & 4 & 23 & 3 & 2 & 11 & & & \\
\hline 190 & 9.93 & $4+14$ & 9.9 & E. $A$ & 34 & 44.45 & 434 & $-34 t$ & 1.44 & $y$ & 25 & 4 & 43.5 & 44.47 & 4 & 2 & I & 7 & $1)$ & & & \\
\hline 160 & 18.96 & 216 & -1.7 & 9.43 & 3 & 16. $\mathrm{F}$ & stou & 0.60 & 1.46 & 5 & 24 & 4 & 14. & $9 . \mathrm{H}$ & 1 & 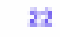 & j & 7 & 11 & & & \\
\hline 170 & ㄹ. 10 & 34 & 1. 19 & $=.34$ & 34 & $2 . \mathrm{Bu}$ & 251 & 1.73 & a. 40 & 3 & 26 & 4 & 2.20 & $=0$ & 1 & $2 y$ & 4 & 19 & 14 & & & \\
\hline 140 & 10.34 & 37 & -4.91 & D. B & 43 & 14 . b4 & 917 & zat & $1.4 \%$ & 6 & 26 & 5 & 19.5 & $=.34$ & I & $2 y$ & 4 & 11 & 17 & & & \\
\hline 180 & 7.94 & 234 & a. 50 & $=.8$ & 42 & 12.62 & 265 & - b.t. & 2.36 & 12 & yt & b & 7.94 & $=.41$ & I & 27 & 4 & 12 & 17 & & & \\
\hline 140 & 4.90 & 34 & a. $=4$ & $=4.44$ & 44 & 10.75 & 911 & bet & d. $4 b$ & ib & 90 & 7 & 4.54 & 9.57 & 12 & 34 & 9 & 11 & 17 & & & \\
\hline 130 & 7.24 & $2 \mathrm{at}$ & 9.21 & $=.47$ & st & 4.15 & 24 & $-1.7 t$ & 0.95 & 13 & $4 b$ & 4 & 7.97 & $=.45$ & $j i$ & 34 & 7 & 11 & 22 & & & \\
\hline t1260 & 4.97 & 240 & 9.93 & : Tis & 5 & B. Fe & 272 & -1.75 & 0.71 & 13 & 36 & 11 & 4.t.t. & 9.44 & 11 & 44 & 9 & 11 & 14 & & & \\
\hline 110 & 5.11 & 216 & 9.롱 & P. $\mathrm{AL}$ & T4 & 4. 들 & 281 & -203 & 0.4 4 & 13 & $t b$ & it & 3.44 & E. At & 12 & 4 & 9 & 11 & 22 & & & \\
\hline cob & 4.91 & 246 & 9.21 & : $\mathrm{AH}$ & Bi & 40 & $27 t$ & -3.00 & a.ti & 13 & +7 & 14 & 3.21 & = $=.74$ & 11 & 57 & 11 & 11 & 27 & & & \\
\hline 40 & 5.13 & $2 x$ & 9.21 & $=.73$ & 123 & 13.늘 & 324 & -8.34 & 2.47 & 13 & $\mathrm{dot}$ & 14 & 3. 14 & D.t. & 11 & $\mathbf{B t}$ & 13 & 11 & 3 & & & \\
\hline 10 & 5.36 & 244 & 9.14 & $=.77$ & 1 th & B. & $2 k i$ & $-2.4 b$ & D. 42 & 13 & 191 & 13 & 4.54 & $=.7 y$ & 11 & 10 & 14 & 11 & 21 & & & \\
\hline 70 & 5.34 & 266 & 9.2H & $=. \mathrm{BL}$ & 157 & T. .52 & 274 & -2.73 & 0.74 & 13 & 195 & 16 & 4.6 & 9.57 & 11 & 121 & 15 & 11 & $\mathrm{st}$ & & & \\
\hline 40 & 4.12 & 290 & -4.6 & $=.4 \mathrm{i}$ & $1 T t$ & B.y & 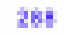 & -1.72 & d.th & 13 & 206 & 16 & 3.7.7 & 9.51 & 11 & it) & 17 & 13 & 24 & & & \\
\hline 30 & 9.31 & 247 & -9.21 & 9.TH & 242 & B. 41 & 27 & -0.40 & t.ts & 13 & 235 & 14 & 3.44 & 9.57 & 11 & 20 & 17 & 13 & $\mathrm{~A}$ & & & \\
\hline 40 & 9.17 & 31 & -4.3 & 4.62 & 134 & 6.t. & 30 te & 10.43 & 4.95 & 13 & 90 & 16 & 3.13 & $=.44$ & 11 & 25 & 17 & 11 & 24 & & & \\
\hline 10 & 4.91 & 246 & -4.94 & $=.64$ & 442 & 5.63 & 265 & -0.43 & 4.91 & 13 & -91 & 16 & 4.14 & 9.57 & 1) & 414 & 19 & 11 & 24 & & & \\
\hline 20 & 2. 76 & 24 & -9.13 & D. AH & Abt & 5.44 & 243 & -0.35 & 4. $9 k$ & 13 & A1 & 21 & ㄹ. 새 & : 14 & 11 & 474 & 17 & 11 & 31 & & & \\
\hline 10 & 4.48 & 141 & a.s & $=.79$ & 74 & 10.6 & 164 & -4.44 & 0.44 & 12: & 497 & 6 & -1.94 & 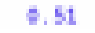 & 1 & Idest & 4 & 11 & 7 & & & \\
\hline
\end{tabular}




\begin{tabular}{|c|c|c|c|}
\hline \multicolumn{4}{|c|}{ ine 1} \\
\hline 1 & \multicolumn{3}{|l|}{ Site Name } \\
\hline 2 & \multicolumn{3}{|l|}{ Start Date/Time } \\
\hline 3 & \multirow{2}{*}{\multicolumn{3}{|c|}{$\begin{array}{l}\text { End Date/Time } \\
\text { SW version }\end{array}$}} \\
\hline 4 & & & \\
\hline 5 & freq & Transmit frequency & $\mathrm{Hz}$ \\
\hline 6 & bw & Filter bandwidth & $\mathrm{Hz}$ \\
\hline 7 & damp & \% Amplitude level & \& \\
\hline 8 & pulw & Transmit pulsewidth & millisecond \\
\hline 9 & rise & Pulse shading & millisecond \\
\hline 10 & rafs & In phasea?set & millivolt \\
\hline 11 & jofs & Quadrature o?set & millivolt \\
\hline \multicolumn{4}{|c|}{ Line 2} \\
\hline 1 & $\sec$ & Wind table time reporting interval & seconds \\
\hline 2 & avdst & Wind table altitude reportinginterval & meters \\
\hline 3 & amp & Fixed amplitude threshold & millivolt \\
\hline 4 & snr & Signal to Noise threshold & \\
\hline 5 & back & Noise samplecollectiontime & millisecond \\
\hline 6 & noms & Not used & \\
\hline 7 & nwt & Not used & \\
\hline 8 & gd & Percent good threshold & \% \\
\hline 9 & $n \pi t$ & Number of FFT points & \\
\hline 10 & srate & Digital samplingrate & $\mathrm{Hz}$ \\
\hline 11 & clut & Ground clutter rejection?ag & \\
\hline 12 & nbini & Signal searchwindow & \# points \\
\hline 13 & ngav & Number of pulsesfor gust detection & \\
\hline 14 & mincr & C Beam spectra searchlimit (lower) & $\mathrm{radial} \mathrm{m} / \mathrm{s}$ \\
\hline 15 & $\operatorname{maxcr}$ & C Beam spectrasearchlimit (upper) & $\mathrm{radial} \mathrm{m} / \mathrm{s}$ \\
\hline 16 & $\operatorname{minbr}$ & B Beam spectrasearchlimit (lower) & $\mathrm{radial} \mathrm{m} / \mathrm{s}$ \\
\hline 17 & $\operatorname{maxbr}$ & B Beam spectrasearchlimit (upper) & $\mathrm{radial} \mathrm{m} / \mathrm{s}$ \\
\hline 18 & minar & A Beam spectra searchlimit (lower) & $\mathrm{radial} \mathrm{m} / \mathrm{s}$ \\
\hline 19 & maxar & A Beam spectra searchlimit (upper) & radial $\mathrm{m} / \mathrm{s}$ \\
\hline 20 & wdog & Watchdog timer (enable ?ag) & \\
\hline 21 & mxdel & Mixing height amplitude detectionthreshold & millivolt \\
\hline 22 & ptdir & Sodar referencefram rotation angle & degrees \\
\hline 23 & wmax & Vertical velocity detection threshold & $\mathrm{m} / \mathrm{s}$ \\
\hline 24 & phase & Interelementspacing & \\
\hline 25 & speci & S Ple output interval increment & \\
\hline 26 & specl & S Tle number of levels output & \\
\hline 27 & specm & 5 ?le ?ag to detail number of axesrecorded & \\
\hline 28 & specn & S 7le number of pulsesaverages & \\
\hline 29 & specs & 5 ?le index of ?rst level recorded & \\
\hline 30 & cdia & DFS data axis & \\
\hline 31 & cdid & DFS number of SRATE samplesper level & \\
\hline 32 & cdin & DFS number of pulsesper record & \\
\hline \multicolumn{4}{|c|}{ (5 } \\
\hline 1 & Axes & Number of active beams & \\
\hline 2 & Levels & Number of asamplingaltitudes & \\
\hline 3 & ZenithV & Zenith angle of $\mathrm{V}$ beam & deg \\
\hline 4 & ZenithU & Zenith angle of $U$ beam & deg \\
\hline 5 & Rotation & Sodar antenna rotation angle & deg \\
\hline 6 & Seperation & Deviation of sodar referencefrom orthogonalorientation & deg \\
\hline 7 & mixHit & Detected mixing height & meters \\
\hline 8 & $\mathrm{rmnU}$ & Noise samplefor $X$ beam & millivolt \\
\hline 9 & $\mathrm{rmnV}$ & Noise samplefor $Y$ beam & millivolt \\
\hline 10 & $\mathrm{rmnW}$ & Noise samplerfor Z beam & millivalt \\
\hline 11 & Antenna status & (optional) status of antenna & \\
\hline \multirow[t]{2}{*}{12} & AC status & (optional) of UPS & \\
\hline & (Optionally) & & \\
\hline 13 & AnemometerTemp & (optional) anemometertemperature & $\operatorname{deg} \mathrm{C}$ \\
\hline 14 & Battery Voltage & (optional) ASP battery voltage (DC systemsonly) & 0.25 \\
\hline
\end{tabular}


Other sources (ccny, sit): The two universities CCNY and SIT operate meteorological instrumentation on exposed sites on their campuses. Their data are available from the internet.

DCNet NOAA (lbr1, eml): Two stations are operated by NOAA. The data were collected from NOAA by internet connection. The files have names such as eml_nyc002_rooftop.txt and the first lines in a file look like the example below:

\begin{tabular}{|c|c|c|c|c|c|c|}
\hline Date & Time & tempc, & relHumidity, & windSpeedMs, & windDir, & baromb \\
\hline $2005-08-01$ & $\odot \odot: 15: \odot \odot$, & 21.5 & 86 & 1.814, & 101 & -999.0 \\
\hline $2005-08-01$ & $\odot \odot: 3 \odot: \odot \odot$, & 21.7, & 84, & 1.661, & 110, & -999.0 \\
\hline $2005-08-01$ & $\odot \odot: 45: \odot \odot$, & 21.6, & 84, & 1.747, & 114, & -999.0 \\
\hline
\end{tabular}

These records are produced each 15 minutes. 


\section{Appendix A: Listing of All Folders and Files In Level 0 for MID05}

The following lists the folder and file names for all level 0 data. A complete list of folders is found

FOLDER: /Volumes/hd1/mid_level0/ccny:

total 752

-rwxrwxrwx 1 rmr rmr 381316 Sep 122005 ccny_08-26-31.txt

FOLDER: /Volumes/hd1/mid_level0/cpk_Central_Park_asos:

total 912

-rwxrwxrwx 1 rmr rmr 460493 Sep 122005 cpk2005.dat

FOLDER: /Volumes/hd1/mid_level0/eml_nyc002:

total 304

-rwxrwxrwx 1 rmr rmr 147512 Sep 122005 eml_nyc002_rooftop.txt

FOLDER: /Volumes/hd1/mid_level0/ewr_NewarkAirport_asos:

total 912

-rwxrwxrwx 1 rmr rmr 460849 Sep 122005 ewr2005.dat

FOLDER: /Volumes/hd1/mid_level0/gm1_GMBldg_roof_met:

total 5166064

-rwxrwxrwx 1 rmr rmr 26519888 Sep 292005 UDP01\$20050804.dat

-rwxrwxrwx 1 rmr rmr 58751448 Aug 62005 UDP01\$20050805.dat

-rwxrwxrwx $1 \mathrm{rmr}$ rmr 58751616 Aug 72005 UDP01\$20050806.dat

-rwxrwxrwx $1 \mathrm{rmr}$ rmr 58751968 Aug 82005 UDP01\$20050807.dat

-rwxrwxrwx $1 \mathrm{rmr}$ rmr 58751984 Aug 92005 UDP01\$20050808.dat

-rwxrwxrwx 1 rmr rmr 58751976 Aug 102005 UDP01\$20050809.dat

-rwxrwxrwx 1 rmr rmr 58751992 Aug 112005 UDP01\$20050810.dat

-rwxrwxrwx $1 \mathrm{rmr}$ rmr 58751952 Aug 122005 UDP01\$20050811.dat

-rwxrwxrwx 1 rmr rmr 33536795 Aug 172005 UDP01\$20050812.dat

-rwxrwxrwx 1 rmr rmr 14157378 Aug 182005 UDP01\$20050817.dat

-rwxrwxrwx $1 \mathrm{rmr}$ rmr 58751944 Aug 192005 UDP01\$20050818.dat

-rwxrwxrwx 1 rmr rmr 58751984 Aug 202005 UDP01\$20050819.dat

-rwxrwxrwx $1 \mathrm{rmr}$ rmr 58752000 Aug 212005 UDP01\$20050820.dat

-rwxrwxrwx 1 rmr rmr 58752001 Sep 302005 UDP01\$20050821.dat

-rwxrwxrwx 1 rmr rmr 58751968 Aug 232005 UDP01\$20050822.dat

-rwxrwxrwx 1 rmr rmr 58750001 Aug 242005 UDP01\$20050823.dat

-rwxrwxrwx 1 rmr rmr 58751952 Aug 252005 UDP01\$20050824.dat

-rwxrwxrwx $1 \mathrm{rmr}$ rmr 58752000 Aug 262005 UDP01\$20050825.dat

-rwxrwxrwx 1 rmr rmr 58751960 Aug 272005 UDP01\$20050826.dat

-rwxrwxrwx 1 rmr rmr 58752000 Aug 282005 UDP01\$20050827.dat

-rwxrwxrwx 1 rmr rmr 58751912 Aug 292005 UDP01\$20050828.dat 
-rwxrwxrwx 1 rmr rmr 58752000 Aug 302005 UDP01\$20050829.dat -rwxrwxrwx 1 rmr rmr 58751912 Aug 312005 UDP01\$20050830.dat -rwxrwxrwx 1 rmr rmr 58751928 Oct 12005 UDP01\$20050831.dat -rwxrwxrwx $1 \mathrm{rmr}$ rmr 32435976 Sep 12005 UDP01\$20050901.dat -rwxrwxrwx $1 \mathrm{rmr}$ rmr 2312 Oct 12005 UDP01x\$20050831.dat -rw-r--r-- 1 rmr rmr 26115128 Sep 302005 gm1_20050804.da1 -rw-r--r-- 1 rmr rmr 57852157 Sep 302005 gm1_20050805.da1 -rw-r--r-- 1 rmr rmr 57870448 Sep 302005 gm1_20050806.da1 -rw-r--r-- 1 rmr rmr 57870582 Sep 302005 gm1_20050807.da1 -rw-r--r-- 1 rmr rmr 57509251 Sep 302005 gm1_20050808.da1 -rW-r--r-- 1 rmr rmr 57811488 Sep 302005 gm1_20050809.da1 -rw-r--r-- 1 rmr rmr 57643921 Sep 302005 gm1_20050810.da1 -rw-r--r-- 1 rmr rmr 57796949 Sep 302005 gm1_20050811.da1 -rw-r--r-- 1 rmr rmr 32787390 Sep 302005 gm1_20050812.da1 -rw-r--r-- 1 rmr rmr 13923406 Sep 302005 gm1_20050817.da1 -rw-r--r-- 1 rmr rmr 57279240 Sep 302005 gm1_20050818.da1 -rw-r--r-- 1 rmr rmr 54208295 Sep 302005 gm1_20050819.da1 -rw-r--r-- 1 rmr rmr 57538061 Sep 302005 gm1_20050820.da1 -rw-r--r-- 1 rmr rmr 57704757 Oct 12005 gm1_20050821.da1 -rW-r--r-- 1 rmr rmr 57388651 Oct 12005 gm1_20050822.da1 -rW-r--r-- 1 rmr rmr 57279174 Oct 12005 gm1_20050823.da1 -rw-r--r-- 1 rmr rmr 56977204 Oct 12005 gm1_20050824.da1 -rw-r--r-- 1 rmr rmr 56747394 Oct 12005 gm1_20050825.da1 -rw-r--r-- 1 rmr rmr 57456589 Oct 12005 gm1_20050826.da1 -rw-r--r-- 1 rmr rmr 57635144 Oct 12005 gm1_20050827.da1 -rw-r--r-- 1 rmr rmr 56754563 Oct 12005 gm1_20050828.da1 -rw-r--r-- 1 rmr rmr 57062897 Oct 12005 gm1_20050829.da1 -rw-r--r-- 1 rmr rmr 56527165 Oct 12005 gm1_20050830.da1 -rW-r--r-- 1 rmr rmr 55021969 Oct 12005 gm1_20050831.da1 -rw-r--r-- 1 rmr rmr 31640417 Oct 12005 gm1_20050901.da1

FOLDER: /Volumes/hd1/mid_level0/gm2_GMBldg_roof_sodar: total 18704

-rwxrwxrwx 1 rmr rmr 231329 Sep 22005 050819dv.dat -rwxrwxrwx 1 rmr rmr 774720 Sep 22005 050820dv.dat -rwxrwxrwx 1 rmr rmr 774720 Sep 22005 050821dv.dat -rwxrwxrwx $1 \mathrm{rmr}$ rmr 774720 Sep 22005 050822dv.dat -rwxrwxrwx 1 rmr rmr 774720 Sep 22005 050823dv.dat -rwxrwxrwx $1 \mathrm{rmr}$ rmr 774720 Sep 22005 050824dv.dat -rwxrwxrwx 1 rmr rmr 774720 Sep 22005 050825dv.dat -rwxrwxrwx 1 rmr rmr 774720 Sep 22005 050826dv.dat -rwxrwxrwx 1 rmr rmr 774720 Sep 22005 050827dv.dat -rwxrwxrwx 1 rmr rmr 774720 Sep 22005 050828dv.dat -rwxrwxrwx $1 \mathrm{rmr}$ rmr 774720 Sep 22005 050829dv.dat -rwxrwxrwx $1 \mathrm{rmr}$ rmr 774720 Sep 22005 050830dv.dat -rwxrwxrwx 1 rmr rmr 774720 Sep 22005 050831dv.dat 
FOLDER: /Volumes/hd1/mid_level0/hpn_airport_asos:

total 2048

-rwxrwxrwx 1 rmr rmr 578254 Sep 122005 hpn2004.dat

-rwxrwxrwx 1 rmr rmr 460298 Sep 122005 hpn2005.dat

FOLDER: /Volumes/hd1/mid_level0/isp_IslipAirp[ort_asos:

total 2272

-rwxrwxrwx $1 \mathrm{rmr}$ rmr 692959 Sep 122005 isp2004.dat

-rwxrwxrwx 1 rmr rmr 460928 Sep 122005 isp2005.dat

FOLDER: /Volumes/hd1/mid_level0/jfk_KennedyAirport_asos:

total 912

-rwxrwxrwx 1 rmr rmr 460927 Sep 122005 jfk2005.dat

FOLDER: /Volumes/hd1/mid_level0/lbr1_BldgA_roof:

total 288

-rwxrwxrwx 1 rmr rmr 141045 Sep 122005 lbr1_BldgA.txt

FOLDER: /Volumes/hd1/mid_level0/lbr2_LehmanBros_setback:

total 4931584

-rwxrwxrwx 1 rmr rmr 16742912 Aug 112005 UDP21\$20050810.dat

-rwxrwxrwx $1 \mathrm{rmr}$ rmr 58788506 Aug 122005 UDP21\$20050811.dat

-rwxrwxrwx 1 rmr rmr 58790377 Aug 132005 UDP21\$20050812.dat

-rwxrwxrwx $1 \mathrm{rmr}$ rmr 58787268 Aug 142005 UDP21\$20050813.dat

-rwxrwxrwx 1 rmr rmr 58823800 Aug 152005 UDP21\$20050814.dat

-rwxrwxrwx 1 rmr rmr 58782440 Aug 162005 UDP21\$20050815.dat

-rwxrwxrwx 1 rmr rmr 58826448 Aug 172005 UDP21\$20050816.dat

-rwxrwxrwx $1 \mathrm{rmr}$ rmr 58786745 Aug 182005 UDP21\$20050817.dat

-rwxrwxrwx 1 rmr rmr 58827418 Aug 192005 UDP21\$20050818.dat

-rwxrwxrwx 1 rmr rmr 58783105 Aug 202005 UDP21\$20050819.dat

-rwxrwxrwx 1 rmr rmr 58825712 Aug 212005 UDP21\$20050820.dat

-rwxrwxrwx $1 \mathrm{rmr}$ rmr 58788004 Aug 222005 UDP21\$20050821.dat

-rwxrwxrwx $1 \mathrm{rmr}$ rmr 58788479 Aug 232005 UDP21\$20050822.dat

-rwxrwxrwx 1 rmr rmr 58827744 Aug 242005 UDP21\$20050823.dat

-rwxrwxrwx 1 rmr rmr 58784590 Aug 252005 UDP21\$20050824.dat

-rwxrwxrwx 1 rmr rmr 58825308 Aug 262005 UDP21\$20050825.dat

-rwxrwxrwx $1 \mathrm{rmr}$ rmr 58785581 Aug 272005 UDP21\$20050826.dat

-rwxrwxrwx $1 \mathrm{rmr}$ rmr 58784484 Aug 282005 UDP21\$20050827.dat

-rwxrwxrwx 1 rmr rmr 58826539 Aug 292005 UDP21\$20050828.dat

-rwxrwxrwx 1 rmr rmr 58786189 Aug 302005 UDP21\$20050829.dat

-rwxrwxrwx 1 rmr rmr 58788460 Aug 312005 UDP21\$20050830.dat

-rwxrwxrwx $1 \mathrm{rmr}$ rmr 58785054 Sep 12005 UDP21\$20050831.dat

-rwxrwxrwx 1 rmr rmr 31369560 Sep 12005 UDP21\$20050901.dat

-rw-r--r-- 1 rmr rmr 16435999 Oct 12005 lbr2_20050810.da1

-rw-r--r-- 1 rmr rmr 57721588 Oct 12005 lbr2_20050811.da1 
-rw-r--r-- 1 rmr rmr 57727868 Oct 12005 lbr2_20050812.da1 -rw-r--r-- 1 rmr rmr 57717684 Oct 12005 lbr2_20050813.da1 -rW-r--r-- 1 rmr rmr 47963224 Oct 12005 lbr2_20050814.da1 -rw-r--r-- 1 rmr rmr 55467385 Oct 12005 lbr2_20050815.da1 -rw-r--r-- 1 rmr rmr 57751951 Oct 12005 lbr2_20050816.da1 -rW-r--r-- 1 rmr rmr 57715623 Oct 12005 lbr2_20050817.da1 -rw-r--r-- 1 rmr rmr 57755408 Oct 12005 lbr2_20050818.da1 -rw-r--r-- 1 rmr rmr 52832185 Oct 12005 lbr2_20050819.da1 -rw-r--r-- 1 rmr rmr 57749827 Oct 12005 lbr2_20050820.da1 -rw-r--r-- 1 rmr rmr 57719599 Oct 12005 lbr2_20050821.da1 -rw-r--r-- 1 rmr rmr 57721380 Oct 12005 lbr2_20050822.da1 -rw-r--r-- 1 rmr rmr 57756437 Oct 12005 lbr2_20050823.da1 -rw-r--r-- 1 rmr rmr 57708718 Oct 12005 lbr2_20050824.da1 -rw-r--r-- 1 rmr rmr 57748099 Oct 12005 lbr2_20050825.da1 -rW-r--r-- 1 rmr rmr 57712021 Oct 12005 lbr2_20050826.da1 -rw-r--r-- 1 rmr rmr 57708347 Oct 12005 lbr2_20050827.da1 -rw-r--r-- 1 rmr rmr 57746510 Oct 12005 lbr2_20050828.da1 -rw-r--r-- 1 rmr rmr 57713725 Oct 12005 lbr2_20050829.da1 -rw-r--r-- 1 rmr rmr 57721306 Oct 12005 lbr2_20050830.da1 -rw-r--r-- 1 rmr rmr 56984425 Oct 12005 lbr2_20050831.da1 -rw-r--r-- 1 rmr rmr 30799234 Oct 12005 lbr2_20050901.da1

FOLDER: /Volumes/hd1/mid_level0/lga_laguardia airport_asos: total 912 -rwxrwxrwx 1 rmr rmr 460847 Sep 122005 lga2005.dat

FOLDER: /Volumes/hd1/mid_level0/metl1_met: total 5697008

-rwxrwxrwx $1 \mathrm{rmr}$ rmr $40800 \mathrm{Jul} 282005$ UDP10\$20050728.dat -rwXrwXrwx 1 rmr rmr 28883642 Jul 302005 UDP10\$20050729.dat -rwxrwxrwx $1 \mathrm{rmr}$ rmr 58542976 Jul 312005 UDP10\$20050730.dat -rwxrwxrwx 1 rmr rmr 58543088 Aug 12005 UDP10\$20050731.dat -rwxrwxrwx 1 rmr rmr 58543752 Aug 22005 UDP10\$20050801.dat -rwxrwxrwx 1 rmr rmr 58543560 Aug 32005 UDP10\$20050802.dat -rwxrwxrwx $1 \mathrm{rmr}$ rmr 58340176 Aug 42005 UDP10\$20050803.dat -rwxrwxrwx 1 rmr rmr 58585784 Aug 52005 UDP10\$20050804.dat -rwxrwxrwx 1 rmr rmr 58544896 Aug 62005 UDP10\$20050805.dat -rwxrwxrwx $1 \mathrm{rmr}$ rmr 58544816 Aug 72005 UDP10\$20050806.dat -rwxrwXrwx 1 rmr rmr 58544864 Aug 82005 UDP10\$20050807.dat -rwxrwxrwx 1 rmr rmr 58544824 Aug 92005 UDP10\$20050808.dat -rwxrwxrwx 1 rmr rmr 58585664 Aug 102005 UDP10\$20050809.dat -rwXrwxrwx 1 rmr rmr 57523736 Aug 112005 UDP10\$20050810.dat -rwXrwXrwx 1 rmr rmr 58585688 Aug 122005 UDP10\$20050811.dat -rwXrwxrwx 1 rmr rmr 21460496 Aug 122005 UDP10\$20050812.dat -rwxrwxrwx 1 rmr rmr 22602129 Aug 142005 UDP10\$20050814.dat -rwxrwxrwx 1 rmr rmr 20399507 Aug 172005 UDP21\$20050817.dat 
-rwxrwxrwx 1 rmr rmr 58751836 Aug 192005 UDP21\$20050818.dat -rwxrwxrwx 1 rmr rmr 58751784 Aug 192005 UDP21\$20050819.dat -rwxrwxrwx 1 rmr rmr 58751834 Aug 202005 UDP21\$20050820.dat -rwxrwxrwx $1 \mathrm{rmr}$ rmr 58751796 Aug 212005 UDP21\$20050821.dat -rwxrwxrwx $1 \mathrm{rmr}$ rmr 58751340 Aug 222005 UDP21\$20050822.dat -rwxrwxrwx 1 rmr rmr 6405526 Aug 222005 UDP21\$20050823.dat -rwxrwxrwx 1 rmr rmr 46552410 Aug 242005 UDP21\$20050824.dat -rwxrwxrwx 1 rmr rmr 58751817 Aug 252005 UDP21\$20050825.dat -rwxrwxrwx $1 \mathrm{rmr}$ rmr 58751808 Aug 262005 UDP21\$20050826.dat -rwxrwxrwx $1 \mathrm{rmr}$ rmr 58751739 Aug 272005 UDP21\$20050827.dat -rwxrwxrwx 1 rmr rmr 58751515 Aug 282005 UDP21\$20050828.dat -rwxrwxrwx 1 rmr rmr 49775815 Aug 292005 UDP21\$20050829.dat -rw-r--r-- 1 rmr rmr 40764 Oct 12005 metl1_20050728.da1 -rw-r--r-- 1 rmr rmr 26651020 Oct 12005 metl1_20050729.da1 -rw-r--r-- 1 rmr rmr 55893170 Oct 12005 metl1_20050730.da1 -rw-r--r-- 1 rmr rmr 56090217 Oct 12005 metl1_20050731.da1 -rw-r--r-- 1 rmr rmr 55908446 Oct 12005 metl1_20050801.da1 -rw-r--r-- 1 rmr rmr 55940137 Oct 12005 metl1_20050802.da1 -rw-r--r-- 1 rmr rmr 54981434 Oct 12005 metl1_20050803.da1 -rw-r--r-- 1 rmr rmr 55365411 Oct 12005 metl1_20050804.da1 -rw-r--r-- 1 rmr rmr 55210909 Oct 12005 metl1_20050805.da1 -rw-r--r-- 1 rmr rmr 53594735 Oct 12005 metl1_20050806.da1 -rw-r--r-- 1 rmr rmr 54821639 Oct 12005 metl1_20050807.da1 -rw-r--r-- 1 rmr rmr 55308997 Oct 12005 metl1_20050808.da1 -rw-r--r-- 1 rmr rmr 55356031 Oct 12005 metl1_20050809.da1 -rw-r--r-- 1 rmr rmr 54420979 Oct 12005 metl1_20050810.da1 -rw-r--r-- 1 rmr rmr 57200072 Oct 12005 metl1_20050811.da1 -rw-r--r-- 1 rmr rmr 21144893 Oct 12005 metl1_20050812.da1 -rw-r--r-- 1 rmr rmr 22050800 Oct 12005 metl1_20050814.da1 -rw-r--r-- 1 rmr rmr 20098579 Oct 12005 metl1_20050817.da1 -rw-r--r-- 1 rmr rmr 57886909 Oct 12005 metl1_20050818.da1 -rw-r--r-- 1 rmr rmr 57886758 Oct 12005 metl1_20050819.da1 -rw-r--r-- 1 rmr rmr 57887166 Oct 12005 metl1_20050820.da1 -rw-r--r-- 1 rmr rmr 57870886 Oct 12005 metl1_20050821.da1 -rw-r--r-- 1 rmr rmr 57832551 Oct 12005 metl1_20050822.da1 -rw-r--r-- 1 rmr rmr 6306729 Oct 12005 metl1_20050823.da1 -rw-r--r-- 1 rmr rmr 45795354 Oct 12005 metl1_20050824.da1 -rw-r--r-- 1 rmr rmr 57846162 Oct 12005 metl1_20050825.da1 -rw-r--r-- 1 rmr rmr 57748869 Oct 12005 metl1_20050826.da1 -rw-r--r-- 1 rmr rmr 57816539 Oct 12005 metl1_20050827.da1 -rw-r--r-- 1 rmr rmr 57837592 Oct 12005 metl1_20050828.da1 -rw-r--r-- 1 rmr rmr 49001378 Oct 12005 metl1_20050829.da1

FOLDER: /Volumes/hd1/mid_level0/metl2_sodar: total 224112

-rwxrwxrwx 1 rmr rmr 1688008 Aug 192005 050803dv.dat 
-rWXrWXrWX

-rwXrwXrwX

-rWXrWXrWX

-rwXrwXrwX

-rwXrwXrwX

-rWXrWXrWX

-rWXrWXrWX

-rwXrwXrwX

-rwXrwXrwX

-rwXrwXrwX

-rwXrwXrwX

-rwXrwXrwX

-rwXrwXrwX

-rwXrwXrwX

-rwXrwXrwX

-rwXrwXrwX

-rwXrwXrwX

-rWXrWXrwX

-rwXrwXrwX

-rwXrwXrwX

-rWXrWXrWX

-rWXIWXIWX

-rwXrwXrwX

-rwXrwXrwX

-rWXrWXrWX

-rWXrwXrwX

-rWXrwXrWX

-rwXrwXrwX

1 rmr rmr 4037760 Aug 192005 050804dv.dat

$1 \mathrm{rmr}$ rmr 4037760 Aug 192005 050805dv.dat

1 rmr rmr 4037760 Aug 192005 050806dv.dat

1 rmr rmr 4037760 Aug 192005 050807dv.dat

1 rmr rmr 4037763 Aug 192005 050808dv.dat

$1 \mathrm{rmr}$ rmr 4037760 Aug 192005 050809dv.dat

$1 \mathrm{rmr}$ rmr 4001308 Aug 192005 050810dv.dat

1 rmr rmr 4037767 Aug 192005 050811dv.dat

1 rmr rmr 4037762 Aug 192005 050812dv.dat

1 rmr rmr 4037760 Aug 192005 050813dv.dat

1 rmr rmr 4037760 Aug 192005 050814dv.dat

1 rmr rmr 4037760 Sep 22005 050815dv.dat

$1 \mathrm{rmr}$ rmr 4037760 Sep 22005 050816dv.dat

$1 \mathrm{rmr}$ rmr 4037774 Sep 22005 050817dv.dat

$1 \mathrm{rmr}$ rmr 4037760 Sep $22005050818 d v$.dat

$1 \mathrm{rmr}$ rmr 4037760 Sep 22005 050819dv.dat

1 rmr rmr 4034839 Sep 22005 050820dv.dat

$1 \mathrm{rmr}$ rmr 4037760 Sep $22005050821 d v$.dat

$1 \mathrm{rmr}$ rmr 4037760 Sep 22005 050822dv.dat

1 rmr rmr 4037760 Sep $22005050823 d v$.dat

$1 \mathrm{rmr}$ rmr 4037760 Sep 22005 050824dv.dat

$1 \mathrm{rmr}$ rmr 4037760 Sep 22005 050825dv.dat

$1 \mathrm{rmr}$ rmr 4037760 Sep 22005 050826dv.dat

$1 \mathrm{rmr}$ rmr 4037760 Sep 22005 050827dv.dat

1 rmr rmr 4037760 Sep 22005 050828dv.dat

$1 \mathrm{rmr}$ rmr 4037760 Sep 22005 050829dv.dat

$1 \mathrm{rmr}$ rmr 4037760 Sep 22005 050830dv.dat

$1 \mathrm{rmr}$ rmr 4037760 Sep 22005 050831dv.dat

drwxr-xr-X 29 rmr rmr 986 Oct 272005 metl2_sodar_dfs

FOLDER: /Volumes/hd1/mid_level0/mgh1_McGrawHill_roof: total 3689664

-rwXrwxrwx 1 rmr rmr 856634 Aug 52005 UDP09\$20050805.dat -rwxrwxrwx 1 rmr rmr 41410139 Aug 152005 UDP09\$20050814.dat -rwxrwxrwx 1 rmr rmr 35618328 Aug 152005 UDP09\$20050815.dat -rwxrwxrwx 1 rmr rmr 28395518 Aug 182005 UDP09\$20050817.dat -rwxrwxrwx 1 rmr rmr 58751976 Aug 192005 UDP09\$20050818.dat -rwXrwxrwx 1 rmr rmr 58751944 Aug 202005 UDP09\$20050819.dat -rwxrwxrwx 1 rmr rmr 58751936 Aug 212005 UDP09\$20050820.dat -rwxrwxrwx 1 rmr rmr 58751936 Aug 222005 UDP09\$20050821.dat -rwxrwxrwx 1 rmr rmr 58751872 Aug 232005 UDP09\$20050822.dat -rwXrwxrwx 1 rmr rmr 58751920 Aug 242005 UDP09\$20050823.dat -rwXrwxrwx 1 rmr rmr 58751952 Aug 252005 UDP09\$20050824.dat -rwxrwxrwx 1 rmr rmr 58751968 Aug 262005 UDP09\$20050825.dat -rwxrwxrwx 1 rmr rmr 58751968 Aug 272005 UDP09\$20050826.dat -rwxrwxrwx 1 rmr rmr 58751976 Aug 282005 UDP09\$20050827.dat 
-rwxrwxrwx 1 rmr rmr 58751936 Aug 292005 UDP09\$20050828.dat -rwxrwxrwx 1 rmr rmr 58751992 Aug 302005 UDP09\$20050829.dat -rwxrwxrwx 1 rmr rmr 58751952 Aug 312005 UDP09\$20050830.dat -rwxrwxrwx $1 \mathrm{rmr}$ rmr 58751976 Sep 12005 UDP09\$20050831.dat -rwxrwxrwx $1 \mathrm{rmr}$ rmr 28355984 Sep 12005 UDP09\$20050901.dat -rw-r--r-- 1 rmr rmr 844094 Oct 12005 mgh1_20050805.da1 -rw-r--r-- 1 rmr rmr 39563193 Oct 12005 mgh1_20050814.da1 -rw-r--r-- 1 rmr rmr 34866694 Oct 12005 mgh1_20050815.da1 -rw-r--r-- 1 rmr rmr 27976749 Oct 12005 mgh1_20050817.da1 -rw-r--r-- 1 rmr rmr 57888497 Oct 12005 mgh1_20050818.da1 -rw-r--r-- 1 rmr rmr 54858020 Oct 12005 mgh1_20050819.da1 -rw-r--r-- 1 rmr rmr 57888363 Oct 12005 mgh1_20050820.da1 -rw-r--r-- 1 rmr rmr 57888296 Oct 12005 mgh1_20050821.da1 -rw-r--r-- 1 rmr rmr 57885214 Oct 12005 mgh1_20050822.da1 -rw-r--r-- 1 rmr rmr 57887693 Oct 12005 mgh1_20050823.da1 -rw-r--r-- 1 rmr rmr 57887961 Oct 12005 mgh1_20050824.da1 -rw-r--r-- 1 rmr rmr 57887760 Oct 12005 mgh1_20050825.da1 -rw-r--r-- 1 rmr rmr 57875968 Oct 12005 mgh1_20050826.da1 -rw-r--r-- 1 rmr rmr 57887291 Oct 12005 mgh1_20050827.da1 -rw-r--r-- 1 rmr rmr 55002807 Oct 12005 mgh1_20050828.da1 -rw-r--r-- 1 rmr rmr 57728568 Oct 12005 mgh1_20050829.da1 -rw-r--r-- 1 rmr rmr 57856270 Oct 12005 mgh1_20050830.da1 -rw-r--r-- 1 rmr rmr 54214083 Oct 12005 mgh1_20050831.da1 -rw-r--r-- 1 rmr rmr 27939497 Oct 12005 mgh1_20050901.da1

FOLDER: /Volumes/hd1/mid_level0/mgh2_McGrawHill_setback: total 6102512

-rwxrwxrwx 1 rmr rmr 31251840 Aug 62005 UDP07\$20050805.dat -rwxrwxrwx $1 \mathrm{rmr}$ rmr 58710464 Aug 72005 UDP07\$20050806.dat -rwxrwxrwx 1 rmr rmr 58752000 Aug 82005 UDP07\$20050807.dat -rwxrwxrwx 1 rmr rmr 58751904 Aug 92005 UDP07\$20050808.dat -rwxrwxrwx 1 rmr rmr 58751968 Aug 102005 UDP07\$20050809.dat -rwxrwxrwx $1 \mathrm{rmr}$ rmr 58751952 Aug 112005 UDP07\$20050810.dat -rwxrwxrwx 1 rmr rmr 58751968 Aug 122005 UDP07\$20050811.dat -rwxrwxrwx 1 rmr rmr 58542304 Aug 132005 UDP07\$20050812.dat -rwxrwxrwx 1 rmr rmr 58742024 Aug 142005 UDP07\$20050813.dat -rwxrwxrwx 1 rmr rmr 58750984 Aug 152005 UDP07\$20050814.dat -rwxrwxrwx 1 rmr rmr 58751840 Aug 162005 UDP07\$20050815.dat -rwxrwxrwx 1 rmr rmr 58751960 Aug 172005 UDP07\$20050816.dat -rwxrwxrwx $1 \mathrm{rmr}$ rmr 58710719 Aug 182005 UDP07\$20050817.dat -rwxrwxrwx 1 rmr rmr 58751944 Aug 192005 UDP07\$20050818.dat -rwxrwxrwx $1 \mathrm{rmr}$ rmr 58745720 Aug 202005 UDP07\$20050819.dat -rwxrwxrwx 1 rmr rmr 58740912 Aug 212005 UDP07\$20050820.dat -rwxrwxrwx 1 rmr rmr 58751064 Aug 222005 UDP07\$20050821.dat -rwxrwxrwx 1 rmr rmr 58751928 Aug 232005 UDP07\$20050822.dat -rwxrwxrwx 1 rmr rmr 58751912 Aug 242005 UDP07\$20050823.dat 
-rwxrwxrwx 1 rmr rmr 58751896 Aug 252005 UDP07\$20050824.dat -rwXrwxrwx 1 rmr rmr 58751872 Aug 262005 UDP07\$20050825.dat -rwxrwxrwx 1 rmr rmr 58745264 Aug 272005 UDP07\$20050826.dat -rwXrwxrwx 1 rmr rmr 58741224 Aug 282005 UDP07\$20050827.dat -rwXrwxrwx 1 rmr rmr 58751480 Aug 292005 UDP07\$20050828.dat -rwXrwxrwx 1 rmr rmr 58751888 Aug 302005 UDP07\$20050829.dat -rwxrwxrwx 1 rmr rmr 58751952 Aug 312005 UDP07\$20050830.dat -rwxrwxrwx $1 \mathrm{rmr}$ rmr 58751928 Sep 12005 UDP07\$20050831.dat -rwxrwxrwx 1 rmr rmr 26234360 Sep 12005 UDP07\$20050901.dat -rw-r--r-- 1 rmr rmr 30790682 Oct 12005 mgh2_20050805.da1 -rw-r--r-- 1 rmr rmr 57845885 Oct 12005 mgh2_20050806.da1 -rw-r--r-- 1 rmr rmr 57888564 Oct 12005 mgh2_20050807.da1 -rw-r--r-- 1 rmr rmr 57888229 Oct 12005 mgh2_20050808.da1 -rw-r--r-- 1 rmr rmr 57888430 Oct 12005 mgh2_20050809.da1 -rw-r--r-- 1 rmr rmr 57888296 Oct 12005 mgh2_20050810.da1 -rw-r--r-- 1 rmr rmr 57888430 Oct 12005 mgh2_20050811.da1 -rw-r--r-- 1 rmr rmr 57668536 Oct 12005 mgh2_20050812.da1 -rw-r--r-- 1 rmr rmr 57855064 Oct 12005 mgh2_20050813.da1 -rw-r--r-- 1 rmr rmr 46458699 Oct 12005 mgh2_20050814.da1 -rW-r--r-- 1 rmr rmr 54937616 Oct 12005 mgh2_20050815.da1 -rW-r--r-- 1 rmr rmr 57888430 Oct 12005 mgh2_20050816.da1 -rw-r--r-- 1 rmr rmr 57846823 Oct 12005 mgh2_20050817.da1 -rw-r--r-- 1 rmr rmr 57888363 Oct 12005 mgh2_20050818.da1 -rw-r--r-- 1 rmr rmr 52708325 Oct 12005 mgh2_20050819.da1 -rw-r--r-- 1 rmr rmr 57851513 Oct 12005 mgh2_20050820.da1 -rw-r--r-- 1 rmr rmr 57885415 Oct 12005 mgh2_20050821.da1 -rW-r--r-- 1 rmr rmr 57888296 Oct 12005 mgh2_20050822.da1 -rW-r--r-- 1 rmr rmr 57888229 Oct 12005 mgh2_20050823.da1 -rW-r--r-- 1 rmr rmr 57888296 Oct 12005 mgh2_20050824.da1 -rw-r--r-- 1 rmr rmr 57888095 Oct 12005 mgh2_20050825.da1 -rw-r--r-- 1 rmr rmr 57865918 Oct 12005 mgh2_20050826.da1 -rw-r--r-- 1 rmr rmr 57852384 Oct 12005 mgh2_20050827.da1 -rw-r--r-- 1 rmr rmr 57886822 Oct 12005 mgh2_20050828.da1 -rW-r--r-- 1 rmr rmr 57888162 Oct 12005 mgh2_20050829.da1 -rw-r--r-- 1 rmr rmr 57888363 Oct 12005 mgh2_20050830.da1 -rw-r--r-- 1 rmr rmr 55744095 Oct 12005 mgh2_20050831.da1 -rw-r--r-- 1 rmr rmr 25849030 Oct 12005 mgh2_20050901.da1

FOLDER: /Volumes/hd1/mid_level0/opp_OnePennPlaza_roof_met: total 5513232

-rw-r--r-- 1 rmr rmr 1853 Sep 292005 CleanSummary.dat -rwXrwxrwx 1 rmr rmr 815922 Aug 32005 UDP05\$20050803.dat -rwXrwXrwx $1 \mathrm{rmr}$ rmr 38683 Aug 102005 UDP05\$20050810.dat -rwxrwxrwx 1 rmr rmr 22113237 Aug 122005 UDP05\$20050811.dat -rwxrwxrwx 1 rmr rmr 44104486 Aug 122005 UDP05\$20050812.dat -rwxrwxrwx 1 rmr rmr 36107472 Aug 182005 UDP05\$20050817.dat 
-rwxrwxrwx 1 rmr rmr 58751968 Aug 192005 UDP05\$20050818.dat -rwxrwxrwx 1 rmr rmr 58751896 Aug 202005 UDP05\$20050819.dat -rwxrwxrwx 1 rmr rmr 58751976 Aug 212005 UDP05\$20050820.dat -rwxrwxrwx $1 \mathrm{rmr}$ rmr 58751968 Aug 222005 UDP05\$20050821.dat -rwxrwxrwx $1 \mathrm{rmr}$ rmr 58752000 Aug 232005 UDP05\$20050822.dat -rwxrwxrwx 1 rmr rmr 58751960 Aug 242005 UDP05\$20050823.dat -rwxrwxrwx 1 rmr rmr 58751968 Aug 252005 UDP05\$20050824.dat -rwxrwxrwx 1 rmr rmr 58751968 Aug 262005 UDP05\$20050825.dat -rwxrwxrwx $1 \mathrm{rmr}$ rmr 58751904 Aug 272005 UDP05\$20050826.dat -rwxrwxrwx $1 \mathrm{rmr}$ rmr 58752000 Aug 282005 UDP05\$20050827.dat -rwxrwxrwx 1 rmr rmr 58751984 Aug 292005 UDP05\$20050828.dat -rwxrwxrwx 1 rmr rmr 58751968 Aug 302005 UDP05\$20050829.dat -rwxrwxrwx 1 rmr rmr 58752000 Aug 312005 UDP05\$20050830.dat -rwxrwxrwx $1 \mathrm{rmr}$ rmr 58751976 Sep 12005 UDP05\$20050831.dat -rwxrwxrwx 1 rmr rmr 58751984 Sep 22005 UDP05\$20050901.dat -rwxrwxrwx 1 rmr rmr 58751976 Sep 32005 UDP05\$20050902.dat -rwxrwxrwx $1 \mathrm{rmr}$ rmr 58751944 Sep 42005 UDP05\$20050903.dat -rwxrwxrwx $1 \mathrm{rmr}$ rmr 58751968 Sep 52005 UDP05\$20050904.dat -rwxrwxrwx $1 \mathrm{rmr}$ rmr 58751968 Sep 62005 UDP05\$20050905.dat -rwxrwxrwx 1 rmr rmr 58751976 Sep 72005 UDP05\$20050906.dat -rwxrwxrwx $1 \mathrm{rmr}$ rmr 58751928 Sep 82005 UDP05\$20050907.dat -rwxrwxrwx $1 \mathrm{rmr}$ rmr 58751960 Sep 92005 UDP05\$20050908.dat -rwxrwxrwx $1 \mathrm{rmr}$ rmr 26152800 Sep 92005 UDP05\$20050909.dat -rw-r--r-- 1 rmr rmr 804296 Oct 12005 opp_20050803.da1 -rw-r--r-- 1 rmr rmr 36878 Oct 12005 opp_20050810.da1 -rw-r--r-- 1 rmr rmr 21788159 Oct 12005 opp_20050811.da1 -rw-r--r-- 1 rmr rmr 43455877 Oct 12005 opp_20050812.da1 -rw-r--r-- 1 rmr rmr 35575889 Oct 12005 opp_20050817.da1 -rW-r--r-- 1 rmr rmr 57888497 Oct 12005 opp_20050818.da1 -rW-r--r-- 1 rmr rmr 57796774 Oct 12005 opp_20050819.da1 -rw-r--r-- 1 rmr rmr 57888497 Oct 12005 opp_20050820.da1 -rw-r--r-- 1 rmr rmr 57888430 Oct 12005 opp_20050821.da1 -rw-r--r-- 1 rmr rmr 57888564 Oct 12005 opp_20050822.da1 -rw-r--r-- 1 rmr rmr 57888363 Oct 12005 opp_20050823.da1 -rw-r--r-- 1 rmr rmr 57888497 Oct 12005 opp_20050824.da1 -rW-r--r-- 1 rmr rmr 57888430 Oct 12005 opp_20050825.da1 -rw-r--r-- 1 rmr rmr 57888296 Oct 12005 opp_20050826.da1 -rw-r--r-- 1 rmr rmr 57888564 Oct 12005 opp_20050827.da1 -rw-r--r-- 1 rmr rmr 57841798 Oct 12005 opp_20050828.da1 -rw-r--r-- 1 rmr rmr 57888497 Oct 12005 opp_20050829.da1 -rW-r--r-- 1 rmr rmr 57888564 Oct 12005 opp_20050830.da1 -rw-r--r-- 1 rmr rmr 57780627 Oct 12005 opp_20050831.da1 -rw-r--r-- 1 rmr rmr 57888497 Oct 12005 opp_20050901.da1 -rw-r--r-- 1 rmr rmr 57886822 Oct 12005 opp_20050902.da1 -rw-r--r-- 1 rmr rmr 57888430 Oct 12005 opp_20050903.da1 -rw-r--r-- 1 rmr rmr 57888497 Oct 12005 opp_20050904.da1 
-rw-r--r-- 1 rmr rmr 57888497 Oct 12005 opp_20050905.da1 -rw-r--r-- 1 rmr rmr 57888497 Oct 12005 opp_20050906.da1 -rw-r--r-- 1 rmr rmr 57888363 Oct 12005 opp_20050907.da1 -rw-r--r-- 1 rmr rmr 57888430 Oct 12005 opp_20050908.da1 -rw-r--r-- 1 rmr rmr 25768764 Oct 12005 opp_20050909.da1

FOLDER: /Volumes/hd1/mid_level0/ppza_ParkPlaza_roof: total 8410448

-rwxrwxrwx 1 rmr rmr 32517392 Aug 52005 UDP02\$20050804.dat -rwxrwxrwx 1 rmr rmr 22031719 Aug 162005 UDP02\$20050805.dat -rwxrwxrwx 1 rmr rmr 23786328 Aug 172005 UDP02\$20050816.dat -rwXrwxrwx 1 rmr rmr 58751992 Aug 182005 UDP02\$20050817.dat -rwXrwxrwx 1 rmr rmr 58751992 Aug 192005 UDP02\$20050818.dat -rwXrwxrwx 1 rmr rmr 58752000 Aug 202005 UDP02\$20050819.dat -rwXrwxrwx 1 rmr rmr 58752000 Aug 212005 UDP02\$20050820.dat -rwXrwxrwx 1 rmr rmr 58751992 Aug 222005 UDP02\$20050821.dat -rwXrwxrwx 1 rmr rmr 58752000 Aug 232005 UDP02\$20050822.dat -rwxrwxrwx 1 rmr rmr 58751940 Aug 242005 UDP02\$20050823.dat -rwXrwxrwx 1 rmr rmr 58752000 Aug 252005 UDP02\$20050824.dat -rwxrwxrwx 1 rmr rmr 58751992 Aug 262005 UDP02\$20050825.dat -rwXrwxrwx 1 rmr rmr 58752000 Aug 272005 UDP02\$20050826.dat -rwxrwxrwx $1 \mathrm{rmr}$ rmr 58752000 Aug 282005 UDP02\$20050827.dat -rwxrwxrwx 1 rmr rmr 58751960 Aug 292005 UDP02\$20050828.dat -rwXrwxrwx 1 rmr rmr 58751960 Aug 302005 UDP02\$20050829.dat -rwxrwxrwx 1 rmr rmr 58751920 Aug 312005 UDP02\$20050830.dat -rwxrwxrwx $1 \mathrm{rmr}$ rmr 58751984 Sep 12005 UDP02\$20050831.dat -rwxrwxrwx 1 rmr rmr 58752000 Sep 22005 UDP02\$20050901.dat -rwxrwxrwx 1 rmr rmr 58751992 Sep 32005 UDP02\$20050902.dat -rwxrwxrwx $1 \mathrm{rmr}$ rmr 58751992 Sep 42005 UDP02\$20050903.dat -rwxrwxrwx $1 \mathrm{rmr}$ rmr 58752000 Sep 52005 UDP02\$20050904.dat -rwxrwxrwx $1 \mathrm{rmr}$ rmr 58751960 Sep 62005 UDP02\$20050905.dat -rwxrwxrwx $1 \mathrm{rmr}$ rmr 58752000 Sep 72005 UDP02\$20050906.dat -rwxrwxrwx $1 \mathrm{rmr}$ rmr 58752000 Sep 82005 UDP02\$20050907.dat -rwxrwxrwx 1 rmr rmr 58751976 Sep 92005 UDP02\$20050908.dat -rwxrwxrwx 1 rmr rmr 22725560 Sep 92005 UDP02\$20050909.dat -rw-r--r-- 1 rmr rmr 32039294 Oct 12005 ppz_20050804.da1 -rW-r--r-- 1 rmr rmr 21708028 Oct 12005 ppz_20050805.da1 -rw-r--r-- 1 rmr rmr 23436829 Oct 12005 ppz_20050816.da1 -rw-r--r-- 1 rmr rmr 57888497 Oct 12005 ppz_20050817.da1 -rw-r--r-- 1 rmr rmr 57888497 Oct 12005 ppz_20050818.da1 -rw-r--r-- 1 rmr rmr 55244811 Oct 12005 ppz_20050819.da1 -rw-r--r-- 1 rmr rmr 57888564 Oct 12005 ppz_20050820.da1 -rw-r--r-- 1 rmr rmr 57888497 Oct 12005 ppz_20050821.da1 -rw-r--r-- 1 rmr rmr 57888564 Oct 12005 ppz_20050822.da1 -rw-r--r-- 1 rmr rmr 57888363 Oct 12005 ppz_20050823.da1 -rw-r--r-- 1 rmr rmr 57888564 Oct 12005 ppz_20050824.da1 
-rw-r--r-- 1 rmr rmr 57888497 Oct 12005 ppz_20050825.da1 -rw-r--r-- 1 rmr rmr 57888564 Oct 12005 ppz_20050826.da1 -rw-r--r-- 1 rmr rmr 57888564 Oct 12005 ppz_20050827.da1 -rw-r--r-- 1 rmr rmr 57810844 Oct 12005 ppz_20050828.da1 -rw-r--r-- 1 rmr rmr 57888430 Oct 12005 ppz_20050829.da1 -rw-r--r-- 1 rmr rmr 57887626 Oct 12005 ppz_20050830.da1 -rw-r--r-- 1 rmr rmr 53208949 Oct 12005 ppz_20050831.da1 -rw-r--r-- 1 rmr rmr 57888564 Oct 12005 ppz_20050901.da1 -rw-r--r-- 1 rmr rmr 57888497 Oct 12005 ppz_20050902.da1 -rw-r--r-- 1 rmr rmr 57888497 Oct 12005 ppz_20050903.da1 -rw-r--r-- 1 rmr rmr 57888564 Oct 12005 ppz_20050904.da1 -rw-r--r-- 1 rmr rmr 57888430 Oct 12005 ppz_20050905.da1 -rw-r--r-- 1 rmr rmr 57888564 Oct 12005 ppz_20050906.da1 -rw-r--r-- 1 rmr rmr 57888564 Oct 12005 ppz_20050907.da1 -rw-r--r-- 1 rmr rmr 57888497 Oct 12005 ppz_20050908.da1 -rw-r--r-- 1 rmr rmr 22391830 Oct 12005 ppz_20050909.da1 -rwxrwxrwx 1 rmr rmr 32038979 Sep 132005 ppza_20050804.da1 -rwxrwxrwx $1 \mathrm{rmr}$ rmr 21707659 Sep 132005 ppza_20050805.da1 -rwxrwxrwx 1 rmr rmr 23436460 Sep 132005 ppza_20050816.da1 -rwxrwxrwx 1 rmr rmr 57888128 Sep 132005 ppza_20050817.da1 -rwxrwxrwx 1 rmr rmr 57888128 Sep 132005 ppza_20050818.da1 -rwxrwxrwx 1 rmr rmr 57598360 Sep 132005 ppza_20050819.da1 -rwxrwxrwx 1 rmr rmr 57888195 Sep 132005 ppza_20050820.da1 -rwxrwxrwx 1 rmr rmr 57888128 Sep 132005 ppza_20050821.da1 -rwxrwxrwx 1 rmr rmr 57888195 Sep 132005 ppza_20050822.da1 -rwxrwxrwx 1 rmr rmr 57887994 Sep 132005 ppza_20050823.da1 -rwxrwxrwx 1 rmr rmr 57888195 Sep 132005 ppza_20050824.da1 -rwxrwxrwx 1 rmr rmr 57888128 Sep 132005 ppza_20050825.da1 -rwxrwxrwx 1 rmr rmr 57888195 Sep 132005 ppza_20050826.da1 -rwxrwxrwx 1 rmr rmr 57888195 Sep 132005 ppza_20050827.da1 -rwxrwxrwx 1 rmr rmr 57884408 Sep 132005 ppza_20050828.da1 -rwxrwxrwx 1 rmr rmr 57888061 Sep 132005 ppza_20050829.da1 -rwxrwxrwx 1 rmr rmr 57887797 Sep 132005 ppza_20050830.da1 -rwxrwxrwx $1 \mathrm{rmr}$ rmr 57082076 Sep 132005 ppza_20050831.da1 -rwxrwxrwx 1 rmr rmr 57888195 Sep 132005 ppza_20050901.da1 -rwxrwxrwx 1 rmr rmr 57888128 Sep 132005 ppza_20050902.da1 -rwxrwxrwx $1 \mathrm{rmr}$ rmr 57888128 Sep 132005 ppza_20050903.da1 -rwxrwxrwx $1 \mathrm{rmr}$ rmr 57888195 Sep 132005 ppza_20050904.da1 -rwxrwxrwx 1 rmr rmr 57888061 Sep 132005 ppza_20050905.da1 -rwxrwxrwx 1 rmr rmr 57888195 Sep 132005 ppza_20050906.da1 -rwxrwxrwx 1 rmr rmr 57888195 Sep 132005 ppza_20050907.da1 -rwxrwxrwx 1 rmr rmr 57888128 Sep 132005 ppza_20050908.da1 -rwxrwxrwx 1 rmr rmr 22391461 Sep 132005 ppza_20050909.da1

FOLDER: /Volumes/hd1/mid_level0/s1:

total 367728 
-rwxrwxrwx $1 \mathrm{rmr}$ rmr 856 Sep 212005 CleanSummary.dat -rwxrwxrwx 1 rmr rmr 13259664 Aug 82005 UDP06\$20050808.dat -rwXrwxrwx 1 rmr rmr 16891072 Aug 122005 UDP06\$20050812.dat -rwxrwxrwx 1 rmr rmr 13667672 Aug 142005 UDP06\$20050814.dat -rwxrwxrwx 1 rmr rmr 16564199 Aug 182005 UDP06\$20050818.dat -rwxrwxrwx 1 rmr rmr 16727656 Aug 202005 UDP06\$20050820.dat -rwXrwxrwx 1 rmr rmr 17012970 Aug 242005 UDP06\$20050824.dat -rwxrwxrwx 1 rmr rmr 13258912 Sep 212005 s1_20050808.da1 -rwxrwxrwx 1 rmr rmr 16890716 Sep 212005 s1_20050812.da1 -rwxrwxrwx 1 rmr rmr 13666776 Sep 212005 s1_20050814.da1 -rwxrwxrwx 1 rmr rmr 16562760 Sep 212005 s1_20050818.da1 -rwxrwxrwx 1 rmr rmr 16726912 Sep 212005 s1_20050820.da1 -rwXrwxrwx 1 rmr rmr 17012104 Sep 212005 s1_20050824.da1

FOLDER: /Volumes/hd1/mid_level0/s10: total 349088

-rw-r--r-- 1 rmr rmr 401 Sep 282005 CleanSummary.dat -rwxrwxrwx 1 rmr rmr 13341514 Aug 92005 UDP17\$20050808.dat -rwXrwxrwx 1 rmr rmr 10852730 Aug 122005 UDP17\$20050812.dat -rwxrwxrwx 1 rmr rmr 15463153 Aug 142005 UDP17\$20050814.dat -rwxrwxrwx 1 rmr rmr 16646341 Aug 182005 UDP17\$20050818.dat -rwxrwxrwx 1 rmr rmr 15871194 Aug 202005 UDP17\$20050820.dat -rwxrwxrwx 1 rmr rmr 17829413 Aug 242005 UDP17\$20050824.dat -rW-r--r-- 1 rmr rmr 13144998 Sep 282005 s10_20050808.da1 -rW-r--r-- 1 rmr rmr 10689649 Sep 282005 s10_20050812.da1 -rw-r--r-- 1 rmr rmr 15233184 Sep 282005 s10_20050814.da1 -rW-r--r-- 1 rmr rmr 16400796 Sep 282005 s10_20050818.da1 -rW-r--r-- 1 rmr rmr 15636795 Sep 282005 s10_20050820.da1 -rW-r--r-- 1 rmr rmr 17566261 Sep 282005 s10_20050824.da1

FOLDER: /Volumes/hd1/mid_level0/s11:

total 189744

-rw-r--r-- 1 rmr rmr 200 Sep 282005 CleanSummary.dat -rwxrwxrwx 1 rmr rmr 15503898 Aug 182005 UDP11\$20050818.dat -rwxrwxrwx 1 rmr rmr 16482717 Aug 202005 UDP11\$20050820.dat -rwxrwxrwx 1 rmr rmr 16931692 Aug 242005 UDP11\$20050824.dat -rW-r--r-- 1 rmr rmr 15275330 Sep 282005 s11_20050818.da1 -rW-r--r-- 1 rmr rmr 16236911 Sep 282005 s11_20050820.da1 -rW-r--r-- 1 rmr rmr 16682464 Sep 282005 s11_20050824.da1

FOLDER: /Volumes/hd1/mid_level0/s12:

total 191968

-rw-r--r-- 1 rmr rmr 199 Sep 282005 CleanSummary.dat -rwxrwxrwx 1 rmr rmr 16075148 Aug 182005 UDP15\$20050818.dat -rwxrwxrwx 1 rmr rmr 15503767 Aug 202005 UDP15\$20050820.dat -rwxrwxrwx 1 rmr rmr 17910924 Aug 242005 UDP15\$20050824.dat 
-rw-r--r-- 1 rmr rmr 15838532 Sep 282005 s12_20050818.da1 -rw-r--r-- 1 rmr rmr 15275594 Sep 282005 s12_20050820.da1 -rw-r--r-- 1 rmr rmr 17646728 Sep 282005 s12_20050824.da1

FOLDER: /Volumes/hd1/mid_level0/s2:

total 181360

-rwxrwxrwx $1 \mathrm{rmr}$ rmr 198 Sep 212005 CleanSummary.dat -rwxrwxrwx 1 rmr rmr 12989596 Aug 122005 UDP13\$20050812.dat -rwxrwxrwx 1 rmr rmr 16727851 Aug 82005 UDP15\$20050808.dat -rwxrwxrwx $1 \mathrm{rmr}$ rmr 16727825 Aug 142005 UDP15\$20050814.dat -rwxrwxrwx 1 rmr rmr 16727319 Sep 212005 s2_20050808.da1 -rwxrwxrwx 1 rmr rmr 12937602 Sep 212005 s2_20050812.da1 -rwxrwxrwx 1 rmr rmr 16727590 Sep 212005 s2_20050814.da1

FOLDER: /Volumes/hd1/mid_level0/s3:

total 368608

-rwxrwxrwx $1 \mathrm{rmr}$ rmr 397 Sep 212005 CleanSummary.dat -rwxrwxrwx $1 \mathrm{rmr}$ rmr 32643 Aug 82005 UDP03\$20050808.av1 -rwxrwxrwx $1 \mathrm{rmr}$ rmr 14238080 Aug 82005 UDP03\$20050808.dat -rwxrwxrwx 1 rmr rmr 15339464 Aug 122005 UDP03\$20050812.dat -rwxrwxrwx 1 rmr rmr 16115272 Aug 142005 UDP03\$20050814.dat -rwxrwxrwx $1 \mathrm{rmr}$ rmr 16643872 Aug 182005 UDP03\$20050818.dat -rwxrwxrwx $1 \mathrm{rmr}$ rmr 15624554 Aug 202005 UDP03\$20050820.dat -rwxrwxrwx $1 \mathrm{rmr}$ rmr 16359768 Aug 242005 UDP03\$20050824.dat -rwxrwxrwx 1 rmr rmr 14236208 Sep 212005 s3_20050808.da1 -rwxrwxrwx 1 rmr rmr 15337128 Sep 212005 s3_20050812.da1 -rwxrwxrwx 1 rmr rmr 16113892 Sep 212005 s3_20050814.da1 -rwxrwxrwx 1 rmr rmr 16639328 Sep 212005 s3_20050818.da1 -rwxrwxrwx 1 rmr rmr 15621300 Sep 212005 s3_20050820.da1 -rwxrwxrwx 1 rmr rmr 16358284 Sep 212005 s3_20050824.da1

FOLDER: /Volumes/hd1/mid_level0/s4:

total 363536

-rwxrwxrwx $1 \mathrm{rmr}$ rmr 395 Sep 212005 CleanSummary.dat -rwxrwxrwx $1 \mathrm{rmr}$ rmr 36549 Aug 82005 UDP08\$20050808.av1 -rwxrwxrwx $1 \mathrm{rmr}$ rmr 15789148 Aug 92005 UDP08\$20050808.dat -rwxrwxrwx 1 rmr rmr 16482816 Aug 122005 UDP08\$20050812.dat -rwxrwxrwx $1 \mathrm{rmr}$ rmr 13015099 Aug 142005 UDP08\$20050814.dat -rwxrwxrwx 1 rmr rmr 13545331 Aug 182005 UDP08\$20050818.dat -rwxrwxrwx $1 \mathrm{rmr}$ rmr 16727736 Aug 202005 UDP08\$20050820.dat -rwxrwxrwx 1 rmr rmr 17461950 Aug 242005 UDP08\$20050824.dat -rwxrwxrwx 1 rmr rmr 15788240 Sep 212005 s4_20050808.da1 -rwxrwxrwx 1 rmr rmr 16481908 Sep 212005 s4_20050812.da1 -rwxrwxrwx 1 rmr rmr 13014724 Sep 212005 s4_20050814.da1 -rwxrwxrwx 1 rmr rmr 13544648 Sep 212005 s4_20050818.da1 -rwxrwxrwx 1 rmr rmr 16727116 Sep 212005 s4_20050820.da1 
-rwxrwxrwx 1 rmr rmr 17460700 Sep 212005 s4_20050824.da1

FOLDER: /Volumes/hd1/mid_level0/s5:

total 367104

-rwxrwxrwx $1 \mathrm{rmr}$ rmr 394 Sep 212005 CleanSummary.dat

-rwxrwxrwx 1 rmr rmr 36921 Aug 82005 UDP04\$20050808.av1

-rwxrwxrwx $1 \mathrm{rmr}$ rmr 16197364 Aug 92005 UDP04\$20050808.dat

-rwxrwxrwx $1 \mathrm{rmr}$ rmr 16279011 Aug 122005 UDP04\$20050812.dat

-rwxrwxrwx 1 rmr rmr 13382248 Aug 142005 UDP04\$20050814.dat

-rwxrwxrwx $1 \mathrm{rmr}$ rmr 16115554 Aug 182005 UDP04\$20050818.dat

-rwxrwxrwx 1 rmr rmr 15707802 Aug 202005 UDP04\$20050820.dat

-rwxrwxrwx 1 rmr rmr 16238161 Aug 242005 UDP04\$20050824.dat

-rwxrwxrwx 1 rmr rmr 16197056 Sep 212005 s5_20050808.da1

-rwxrwxrwx 1 rmr rmr 16278452 Sep 212005 s5_20050812.da1

-rwxrwxrwx 1 rmr rmr 13381856 Sep 212005 s5_20050814.da1

-rwxrwxrwx 1 rmr rmr 16114368 Sep 212005 s5_20050818.da1

-rwxrwxrwx 1 rmr rmr 15707320 Sep 212005 s5_20050820.da1

-rwxrwxrwx 1 rmr rmr 16237584 Sep 212005 s5_20050824.da1

FOLDER: /Volumes/hd1/mid_level0/s6:

total 152496

-rwxrwxrwx $1 \mathrm{rmr}$ rmr 198 Sep 212005 CleanSummary.dat

-rwxrwxrwx $1 \mathrm{rmr}$ rmr 17095168 Aug 122005 UDP11\$20050812.dat

-rwxrwxrwx 1 rmr rmr 13830979 Aug 142005 UDP11\$20050814.dat

-rwxrwxrwx 1 rmr rmr 651 Aug 62005 UDP20\$20050806.av1

-rwxrwxrwx $1 \mathrm{rmr}$ rmr 34596 Aug 82005 UDP20\$20050808.av1

-rwxrwxrwx $1 \mathrm{rmr}$ rmr 8076751 Aug 82005 UDP20\$20050808.dat

-rwxrwxrwx 1 rmr rmr 8074924 Sep 212005 s6_20050808.da1

-rwxrwxrwx 1 rmr rmr 17094520 Sep 212005 s6_20050812.da1

-rwxrwxrwx 1 rmr rmr 13829424 Sep 212005 s6_20050814.da1

FOLDER: /Volumes/hd1/mid_level0/s7:

total 364608

-rwxrwxrwx 1 rmr rmr 651 Aug 42005 UDP12\$20050804.av1

-rwxrwxrwx $1 \mathrm{rmr}$ rmr 15789384 Aug 82005 UDP12\$20050808.dat

-rwxrwxrwx 1 rmr rmr 14687608 Aug 122005 UDP12\$20050812.dat

-rwxrwxrwx $1 \mathrm{rmr}$ rmr 16645967 Aug 142005 UDP12\$20050814.dat

-rwxrwxrwx $1 \mathrm{rmr}$ rmr 15952580 Aug 182005 UDP12\$20050818.dat

-rwxrwxrwx 1 rmr rmr 13627072 Aug 202005 UDP12\$20050820.dat

-rwxrwxrwx 1 rmr rmr 17299032 Aug 242005 UDP12\$20050824.dat

-rwxrwxrwx 1 rmr rmr 15557227 Oct 62005 s7_20050808.da1

-rwxrwxrwx $1 \mathrm{rmr}$ rmr 14471223 Oct 62005 s7_20050812.da1

-rwxrwxrwx $1 \mathrm{rmr}$ rmr 16400684 Oct 62005 s7_20050814.da1

-rwxrwxrwx 1 rmr rmr 15718094 Oct 62005 s7_20050818.da1

-rwxrwxrwx $1 \mathrm{rmr}$ rmr 13426962 Oct 62005 s7_20050820.da1

-rwxrwxrwx 1 rmr rmr 17044761 Oct 62005 s7_20050824.da1 
FOLDER: /Volumes/hd1/mid_level0/s8:

total 363520

-rwxrwxrwx $1 \mathrm{rmr}$ rmr 122361 Aug 122005 (lab)UDP16\$20050812.dat

-rw-r--r-- 1 rmr rmr 453 Sep 282005 CleanSummary.dat

-rwxrwxrwx $1 \mathrm{rmr}$ rmr 837 Aug 42005 UDP16\$20050804.av1

-rwxrwxrwx $1 \mathrm{rmr}$ rmr 36270 Aug 82005 UDP16\$20050808.av1

-rwxrwxrwx 1 rmr rmr 15136656 Aug 82005 UDP16\$20050808.dat

-rwxrwxrwx $1 \mathrm{rmr}$ rmr 13911718 Aug 142005 UDP16\$20050814.dat

-rwxrwxrwx $1 \mathrm{rmr}$ rmr 16687124 Aug 182005 UDP16\$20050818.dat

-rwxrwxrwx 1 rmr rmr 16034242 Aug 202005 UDP16\$20050820.dat

-rwxrwxrwx 1 rmr rmr 17299080 Aug 242005 UDP16\$20050824.dat

-rwxrwxrwx $1 \mathrm{rmr}$ rmr 14565332 Aug 122005 UDP21\$20050812.dat

-rw-r--r-- 1 rmr rmr 14913798 Sep 282005 s8_20050808.da1

-rw-r--r-- 1 rmr rmr 14350245 Sep 282005 s8_20050812.da1

-rw-r--r-- 1 rmr rmr 13705706 Sep 282005 s8_20050814.da1

-rW-r--r-- 1 rmr rmr 16441599 Sep 282005 s8_20050818.da1

-rw-r--r-- 1 rmr rmr 15798196 Sep 282005 s8_20050820.da1

-rw-r--r-- 1 rmr rmr 17044398 Sep 282005 s8_20050824.da1

FOLDER: /Volumes/hd1/mid_level0/s9:

total 324720

-rw-r--r-- 1 rmr rmr 393 Sep 282005 CleanSummary.dat

-rwxrwxrwx $1 \mathrm{rmr}$ rmr 5180728 Aug 82005 UDP13\$20050808.dat

-rwxrwxrwx 1 rmr rmr 13830916 Aug 142005 UDP13\$20050814.dat

-rwxrwxrwx $1 \mathrm{rmr}$ rmr 15299488 Aug 182005 UDP13\$20050818.dat

-rwxrwxrwx $1 \mathrm{rmr}$ rmr 15870864 Aug 202005 UDP13\$20050820.dat

-rwxrwxrwx 1 rmr rmr 16319753 Aug 242005 UDP13\$20050824.dat

-rwxrwxrwx $1 \mathrm{rmr}$ rmr 17217466 Aug 122005 UDP15\$20050812.dat

-rw-r--r-- 1 rmr rmr 5102452 Sep 282005 s9_20050808.da1

-rw-r--r-- 1 rmr rmr 16964065 Sep 282005 s9_20050812.da1

-rw-r--r-- 1 rmr rmr 13626795 Sep 282005 s9_20050814.da1

-rw-r--r-- 1 rmr rmr 15073392 Sep 282005 s9_20050818.da1

-rw-r--r-- 1 rmr rmr 15636594 Sep 282005 s9_20050820.da1

-rW-r--r-- 1 rmr rmr 16078928 Sep 282005 s9_20050824.da1

FOLDER: /Volumes/hd1/mid_level0/sit_Stevens_HoweCenter:

total 416

-rwxrwxrwx 1 rmr rmr 206580 Sep 122005 sit_HoweCenter.txt 


\section{Appendix B: Listing of All Folders and Files for Level 1 Data}

\section{DESCRIPTION OF THE LEVEL 1 DATA FOLDERS AND FILES}

The level 1 data are located in the level1 folder on a path /data/mid/level1. A listing of the level 1 folder is given below.

\begin{tabular}{|c|c|c|c|c|c|c|c|c|}
\hline$d r w \times r-x r-x$ & 7 & $r m r$ & $r m r$ & 238 & Apr & 18 & $13: 25$ & field \\
\hline drwxrwxrwx & 15 & $r m r$ & $r m r$ & 510 & Apr & 6 & $21: 41$ & iop1 \\
\hline$d r w \times r w \times r w x$ & 15 & $r m r$ & $r m r$ & 510 & Apr & 6 & $20: 42$ & iop2 \\
\hline$d r w \times r w \times r w x$ & 15 & $r m r$ & $r m r$ & 510 & Apr & 6 & $22: 42$ & iop3 \\
\hline drwxrwxrwx & 15 & $r m r$ & $r m r$ & 510 & Apr & 6 & $22: 46$ & iop4 \\
\hline drwxrwxrwx & 15 & $r m r$ & $r m r$ & 510 & Apr & 6 & $22: 47$ & iop5 \\
\hline drwxrwxrwx & 15 & $r m r$ & $r m r$ & 510 & Apr & 6 & $22: 48$ & iop6 \\
\hline$d r w \times r w \times r w x$ & 22 & $r m r$ & $r m r$ & 748 & Apr & 6 & $23: 42$ & eso \\
\hline
\end{tabular}

where the folders hold data from the field calibrations, the six IOPs and the mesonet array that was in operation most of the month of August.

\section{Field Data files}

The field data are divided into four directories gp1, gp2, gp3, and gp4. Each directory contains a data set from is a different time when a different set of sonic anemometers were compared.

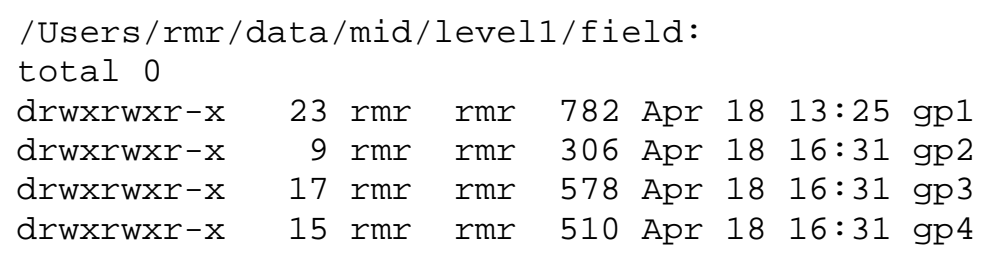

Each of the folders contain avg files for the instruments in that group. As an example, the contents of gp1 is

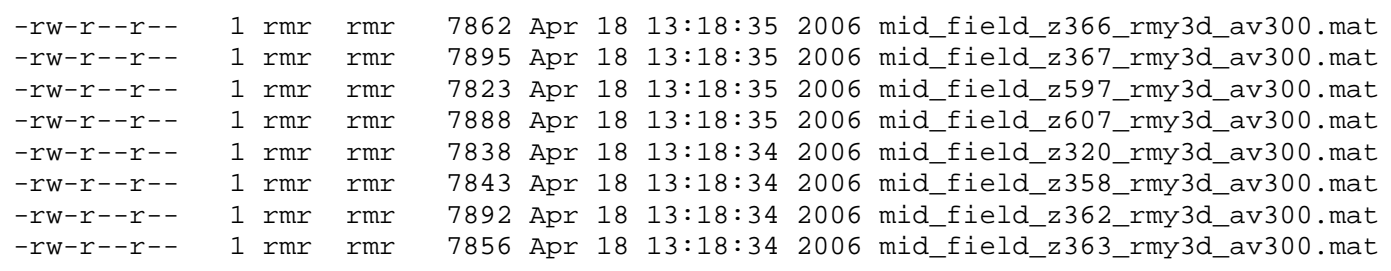




\begin{tabular}{|c|c|c|c|c|c|c|c|c|c|}
\hline$-r W-r--r--$ & 1 & $\mathrm{rmr}$ & $r m r$ & & Apr & 18 & $3: 18: 34$ & 006 & mid_field_z364_rmy3d_av300.mat \\
\hline$-r W-r--r--$ & 1 & $\mathrm{rmr}$ & $r m r$ & 7793 & Apr & 18 & $3: 18: 34$ & 006 & mid_field_z365_rmy3d_av300.mat \\
\hline$-r w-r--r--$ & 1 & $r m r$ & $r m r$ & 37202 & Apr & 17 & $5: 04: 40$ & 006 & $\operatorname{mid} \mathrm{fi}^{-1} \mathrm{~d}^{-} \mathrm{r} 607^{-} \mathrm{r}$ \\
\hline$-r w-r--r--$ & 1 & $\mathrm{rmr}$ & $\mathrm{rmr}$ & 36864 & Apr & 17 & $5: 04: 39$ & 006 & mid_field_z320_rmy3d_av300 \\
\hline$-r w-r--r--$ & 1 & $r m r$ & $r m r$ & 7202 & Apr & 17 & & 006 & $58 r$ \\
\hline$-r w-r--r--$ & 1 & $r m r$ & $r m r$ & 37202 & Apr & 7 & 39 & $\odot \odot 6$ & 2_rmy \\
\hline$-r w-r--r--$ & 1 & $\mathrm{rmr}$ & $r m r$ & 37202 & Apr & 7 & 39 & $\odot \odot 6$ & 63_rn \\
\hline$-r w-r--r--$ & 1 & $\mathrm{rmr}$ & $r m r$ & 37202 & Apr & & 39 & 006 & 34_rmy \\
\hline$-r w-r--r--$ & 1 & $r m r$ & $r m r$ & 36864 & Apr & 7 & 39 & 006 & 5_rmy3d_av30 \\
\hline$r w-r--r--$ & 1 & $\mathrm{rmr}$ & $r m r$ & 372 & Apr & & 39 & 06 & 6_rmy3d_av300 \\
\hline$r w-r--r--$ & 1 & $\mathrm{rmr}$ & $\mathrm{rmr}$ & & Apr & & & & 67_rmy3d_av30 \\
\hline$r w-r--r--$ & 1 & $\mathrm{rmr}$ & $\mathrm{rmr}$ & 37202 & Apr & 17 & $15: \odot 4: 39$ & 2006 & ld_z597_rmy3d_av30 \\
\hline
\end{tabular}

where the file names show the experiment (mid), the data segment (field), the sonic serial number (z366), the instrument type (rmy3d), and the averaging time in seconds (av300). The format of these files is explained below in Appendix B.1 that describes level 1 data formats. Twenty instruments were compared during gp1 time period.

\section{IOP Data Files}

Each of the six IOP data folders is organized in the same way. The iop1 folder contains one ASCII file named INFO_mid_iop1.txt is an information file that provides details about that particular IOP. Any information that is general to the IOP and applies to all measurement systems is recorded in this file. It also contains folders for each street level anemometer used during the IOP. The directory is shown below:

\begin{tabular}{|c|c|c|c|c|c|c|c|c|}
\hline - rwxrwxrwx & 1 & $r m r$ & $r m r$ & 1064 & Sep & 21 & 2005 & INF0_mid_iop1.txt \\
\hline drwxrwxrwx & 5 & $r m r$ & $r m r$ & 170 & Apr & 6 & $21: 39$ & 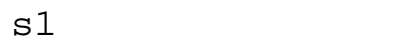 \\
\hline drwxrwxrwx & 5 & $r m r$ & $r m r$ & 170 & Apr & 6 & $21: 39$ & s2 \\
\hline drwxrwxrwx & 5 & $r m r$ & $r m r$ & 170 & Apr & 6 & $21: 39$ & s3 \\
\hline drwxrwxrwx & 5 & $r m r$ & $r m r$ & 170 & Apr & 6 & $21: 39$ & s4 \\
\hline drwxrwxrwx & 5 & $r m r$ & $r m r$ & 170 & Apr & 6 & $21: 39$ & s5 \\
\hline drwxrwxrwx & 5 & $r m r$ & $r m r$ & 170 & Apr & 6 & $21: 39$ & s6 \\
\hline$d r w \times r w \times r w x$ & 5 & $r m r$ & $r m r$ & 170 & Apr & 6 & $21: 39$ & s7 \\
\hline drwxrwxrwx & 5 & $r m r$ & $r m r$ & 170 & Apr & 6 & $21: 39$ & s8 \\
\hline drwxrwxrwx & 5 & $r m r$ & $r m r$ & 170 & Apr & 6 & $21: 39$ & s9 \\
\hline drwxrwxrwx & 5 & $r m r$ & $r m r$ & 170 & Apr & 6 & $21: 41$ & s10 \\
\hline
\end{tabular}

The folders s1, s2, ..., s10 hold all level 1 data for the particular station. One example of this folder, s1, is given below:

\footnotetext{
-rwxrwxrwx $1 \mathrm{rmr}$ rmr $610 \mathrm{Apr} 6$ 22:38 INF0_mid_iop1_s1.txt

-rwxrwxrwX $1 \mathrm{rmr}$ rmr $9224 \mathrm{Apr} 907: 17$ mid_iop1_s1_rmy3d_av300.dat
}

where the file INFO_mid_iop1_s1.txt is an information ASCII file that provides specific information about the station, s1, during IOP 1. Instrument failures, alignment issues, wind blockage from truck, etc are entered to this file. The averaged wind data are listed in the ASCII data file with a name of mid_iop1_s1_rmy3d_av300.dat which includes the experiment (mid), IOP (iop1), station (s1), instrument (rmy3d), and averaging time in seconds (av300).

\section{$\underline{\text { Meso Data Files }}$}


The level 1 folder called "meso" holds the mesoscale wind network data for the experiment. The instruments and data collected here cover the entire month of August as continuous time series. The meso folder is as follows:

\begin{tabular}{|c|c|c|c|c|c|c|c|c|}
\hline - rwxrwxrwx & 1 & $r m r$ & $r m r$ & 591 & Oct & 1 & 2005 & INF0_mid_meso.txt \\
\hline$d r w x r-x r-x$ & 4 & $\mathrm{rmr}$ & $\mathrm{rmr}$ & 136 & Apr & 6 & $23: 05$ & ccny_roof_met \\
\hline drwxrwxrwx & 6 & $\mathrm{rmr}$ & $\mathrm{rmr}$ & 204 & Apr & 6 & $23: 25$ & cpk_CentralPark_asos \\
\hline$d r w x r-x r-x$ & 5 & $r m r$ & $r m r$ & 170 & Apr & 6 & $23: 07$ & eml_EnvironMeasLab_DCnet \\
\hline$d r w x r-x r-x$ & 6 & $\mathrm{rmr}$ & $\mathrm{rmr}$ & 204 & Apr & 6 & $23: 25$ & ewr_Newark_asos \\
\hline drwxrwxrwx & 7 & $r m r$ & $r m r$ & 238 & Apr & 14 & $06: 25$ & gm1_GeneralMotors_met \\
\hline drwxrwxrwx & 42 & $\mathrm{rmr}$ & $r m r$ & 1428 & Apr & 14 & $06: 26$ & gm2_GMBldg_sodar \\
\hline$d r w x r-x r-x$ & 6 & $r m r$ & $r m r$ & 204 & Apr & 6 & $23: 26$ & jfk_Kennedy_asos \\
\hline drwxrwxrwx & 4 & $\mathrm{rmr}$ & $\mathrm{rmr}$ & 136 & Apr & 6 & $23: 28$ & lbr1_LhrBros_roof_noaa \\
\hline drwxrwxrwx & 5 & $r m r$ & $r m r$ & 170 & Oct & 6 & 2005 & Ibr2_LehmanBros_setback \\
\hline drwxrwxrwx & 6 & $r m r$ & $r m r$ & 204 & Apr & 6 & $23: 29$ & lga_LaguardiaAP_asos \\
\hline drwxrwxrwx & 7 & $r m r$ & $r m r$ & 238 & Apr & 14 & $06: 26$ & met1_MetLife_met \\
\hline drwxrwxrwx & 42 & $r m r$ & $r m r$ & 1428 & Apr & 14 & $06: 27$ & met2_MetLife_sodar \\
\hline drwxrwxrwx & 5 & $r m r$ & $r m r$ & 170 & Oct & 4 & 2005 & mgh1_McGraw-Hill_roof \\
\hline drwxrwxrwx & 5 & $r m r$ & $r m r$ & 170 & Oct & 5 & 2005 & mgh2_McGraw-Hill_setback \\
\hline drwxrwxrwx & 5 & $r m r$ & $r m r$ & 170 & Oct & 6 & 2005 & орр \\
\hline drwxrwxrwx & 5 & $r m r$ & $r m r$ & 170 & Oct & 6 & 2005 & ppz_ParkPlaza_roof \\
\hline$d r w x r-x r-x$ & 7 & $r m r$ & $r m r$ & 238 & Apr & 6 & $23: 53$ & sit1_met \\
\hline
\end{tabular}

where the ASCII information file, INFO_mid_meso.txt provides general information about mesonet data. Data specific to a particular station are not in this file.

The different mesonet stations are discussed in the Appendix that describes Level 0 file organization. The following types of stations are included here:

gm1,lbr2,met1,mgh1,mgh2,opp,ppz: UDP rooftop or setback wind stations. These are all RMY 3D sonic anemometers.

gm2,met2: mini Sodar installations.

cpk,ewr,jfk,lga: NOAA NWS ASOS stations.

ccny: The rooftop station from CCNY. This is an important site at the north end of Manhattan.

eml: The NOAA DCnet station on the roof of the DHS's EML building in west Greenwich Village.

lbr1: The NOAA Dcnet station on the roof of Building 'A'

sit1: The meteorological system deployed by the Stevens Institute of Technology in Hoboken New Jersey.

\section{ASOS Data Files}

The Automated Observing Systems (ASOS) weather stations are deployed by NOAA around the country. The ASOS data are collected over the internet (see 
http://www.ncdc.noaa.gov/oa/climate/stationlocator.html) in a standard format. The raw files

from the internet have a format shown below:

Month:, $06 / 2005$

Station Name:, "JOHN F KENNEDY INTERNATIONAL AIRPORT" Call Sign:, JFK

Day, Time, StationType, Maint Indic, SkyConditions, Visibility, weather Type, Dry Bulb Faren, Dry Bulb Cel, wet Bulb Faren, Wet Bulb Cel, Dew Point Faren, Dew Point Cel, Rel Humd, Wind Speed, Wind Dir, Wind

Char Gusts, Val. for Wind Char, Station Pressure, Press Tend, Sea Level Pressure, Report Type, Precip Total

$01,0051, \mathrm{A02},-$ FEWOO8

$12.4,53,11.7,90,5,090,-, \odot, 30.14,0,216, A A,-$

$01,0151, A 02,1,3$

$12.1,52,11.1,87,6,090,-, 0,30.15,-, 218, \mathrm{AA},-$

$01,0251, \mathrm{A02},-, \mathrm{BKN} 008$

$12.4,53,11.7,90,5,090,-, 0,30.14,-, 217, \mathrm{AA},-$

01,0351, A02 , - , OVCO०8

$12.1,52,11.1,87,8,090,-, 0,30.16,3,221, A A,-$

$\begin{array}{lll}, 10 \mathrm{SM} & ,-, 56 \quad, \quad 13.3,54, \\ , 8 \mathrm{SM} & ,-, 56 \quad, \quad 13.3,54 \\ , 7 \mathrm{SM} & ,-, 56 \quad, \quad 13.3,54 \\ , 10 \mathrm{SM} & ,-, 56 \quad, \quad 13.3,54\end{array}$

where the lines are wrapped for convenience. We have ASOS data from 2000 to present day. A

PERL program named ReadNwsAsos.pl reads the raw files and the level 1 files. Level 1 files

with names such as jfk2005.dat where the station identifier (jfk) and the year (2005) define the

data set. An example of the first lines of this file is given below.

PROGRAM: ReadNwSAsos.pl

Version: v1.01, Editdate: 2005/09/12

Station: $j \mathrm{fk}$

Year: 2005

Time correction: 0

DATE (JD) TIME Npts wspd(m/s) $\operatorname{wdir}(\operatorname{deg}) \quad \operatorname{tair}(\mathrm{C}) \quad \operatorname{tdew}(\mathrm{C}) \quad \operatorname{rh}(\%) \quad \operatorname{slp}(\mathrm{hPa}) \quad \mathrm{ppt}(\mathrm{mm})$

2005-02-28 (059) 23:51:00, 5.7, 10, -0.6, -1.7, 92, 996.8, 0.8

2005-03-01 (060) 00:51:00, 5.1, 360, $-0.6, \quad-2.2, \quad 89, \quad 996.5,0.0$

2005-03-01 (060) 01:51:00, 5.7, $10, \quad-0.6, \quad-2.8, \quad 85, \quad 995.8,1.0$

2005-03-01 (060) 02:51:00, 3.6, 350, -1.1, -2.2, 92, 995.8, 0.3

Note that the ASOS data are reported once each hour at 51 minutes after the hour. Each record gives averages for a five-minute sampling period. The data here are taken directly from the raw

files, possibly with a change of units.

\section{$\underline{\text { CCNY Data File Formats }}$}

The CCNY data are available from the BNL mesonet data base.

Timestamp, tempc, relHumidity, windSpeedMs, windDir, baroMb 2005-08-26 12:36:00, 26.9, 34,2.850, 234, 999.9 2005-08-26 12:37:00, 26.7, 35, $0.900,275,999.9$ 2005-08-26 12:38:00, 26.8, 35,1.840, 202, 999.9 2005-08-26 12:39:00, 26.9, 36,2.920, 192, 999.9 2005-08-26 12:40:00, 27.0, 34,3.500, 213, 999.9 2005-08-26 12:41:00, 26.9, 33,2.720, 218, 999.9 
2005-08-26 12:42:00, 26.7, 33,2.060, 224, 999.9

The CCNY data are reported each minute. Units are in metric, relative to true north. The internet files are averaged and converted to level 1 files with a PERL program, wx1_web_avg.pl which is used on all files that are derived from the BNL data base.

The output of the program is a level 1 averaged file with a name such as mid_meso_lbr1_web_av300.dat where the name includes the experiment name (mid), the data segment (meso), the station ID (lbr1), and the averaging time, in seconds (300). An example of the first lines of data follows: 


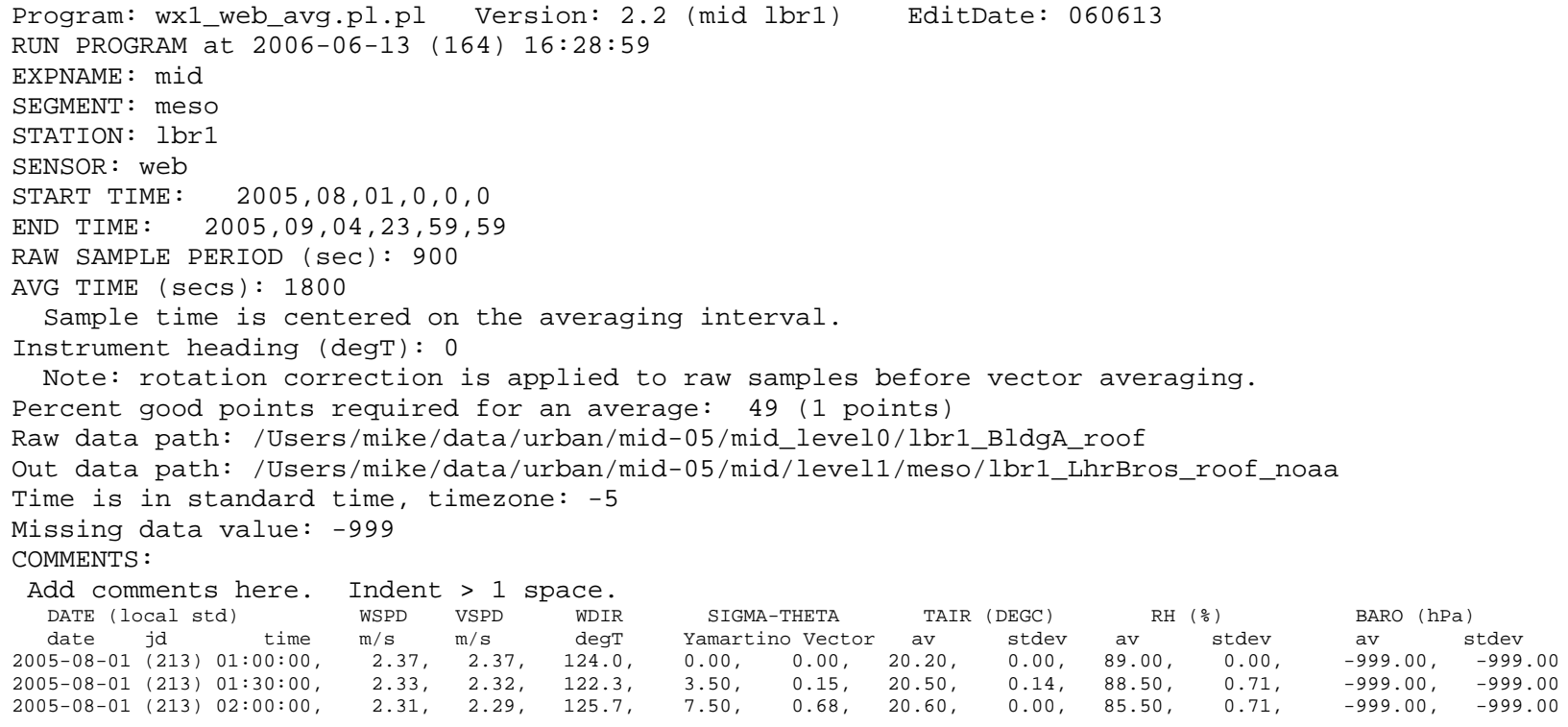

The header lines are wrapped for convenience. The format of the files here is exactly the same for all data sets that are generated from the BNL mesonet web site. 


\section{B.1 FILES CONTAINED IN THE LEVEL 1 FOLDERS}

Folder: /Users/rmr/data/mid/level1/field:

total 0

drwxrwxr-x 23 rmr rmr 782 Apr 18 13:25 gp1

drwxrwxr-x 9 rmr rmr 306 Apr 18 16:31 gp2

drwxrwxr-x 17 rmr rmr 578 Apr 18 16:31 gp3

drwxrwxr-x 15 rmr rmr 510 Apr 18 16:31 gp4

FOLDER/Users/rmr/data/mid/level1/iop1:

total 16

-rw-r--r-- 1 rmr rmr 635 Apr 6 22:38 INFO.tmp

-rwxrwxrwx 1 rmr rmr 1064 Sep 212005 INFO_mid_iop1.txt

drwxrwxrwx $5 \mathrm{rmr}$ rmr 170 Apr 6 21:39 s1

drwxrwxrwx $5 \mathrm{rmr}$ rmr 170 Apr 6 21:41 s10

drwxrwxrwx $5 \mathrm{rmr}$ rmr 170 Apr 6 21:39 s2

drwxrwxrwx $5 \mathrm{rmr}$ rmr 170 Apr 6 21:39 s3

drwxrwxrwx $5 \mathrm{rmr}$ rmr 170 Apr 6 21:39 s4

drwxrwxrwx $5 \mathrm{rmr}$ rmr 170 Apr 6 21:39 s5

drwxrwxrwx $5 \mathrm{rmr}$ rmr 170 Apr 6 21:39 s6

drwxrwxrwx $5 \mathrm{rmr}$ rmr 170 Apr 6 21:39 s7

drwxrwxrwx $5 \mathrm{rmr}$ rmr 170 Apr 6 21:39 s8

drwxrwxrwx $5 \mathrm{rmr}$ rmr 170 Apr 6 21:39 s9

FOLDER /Users/rmr/data/mid/level1/iop2:

total 16

-rw-r--r-- 1 rmr rmr 635 Apr 6 22:42 INFO.tmp

-rwxrwxrwx $1 \mathrm{rmr}$ rmr 689 Sep 212005 INFO_mid_iop2.txt

drwxrwxrwx $4 \mathrm{rmr}$ rmr 136 Oct $62005 \mathrm{~s} 1$

drwxrwxrwx $5 \mathrm{rmr}$ rmr 170 Oct $62005 \mathrm{~s} 10$

drwxrwxrwx $5 \mathrm{rmr}$ rmr 170 Oct $62005 \mathrm{~s} 2$

drwxrwxrwx 4 rmr rmr 136 Oct 62005 s3

drwxrwxrwx $4 \mathrm{rmr}$ rmr 136 Oct $62005 \mathrm{~s} 4$

drwxrwxrwx $4 \mathrm{rmr}$ rmr 136 Oct $62005 \mathrm{~s} 5$

drwxrwxrwx 5 rmr rmr 170 Apr 6 22:43 s6

drwxrwxrwx $5 \mathrm{rmr}$ rmr 170 Oct $62005 \mathrm{~s} 7$

drwxrwxrwx $5 \mathrm{rmr}$ rmr 170 Oct $62005 \mathrm{~s} 8$

drwxrwxrwx 4 rmr rmr 136 Oct 62005 s9

FOLDER /Users/rmr/data/mid/level1/iop3:

total 16

-rw-r--r-- 1 rmr rmr 724 Apr 6 22:45 INFO.tmp

-rwxrwxrwx $1 \mathrm{rmr}$ rmr 595 Apr 9 06:14 INFO_mid_iop3.txt

drwxrwxrwx 5 rmr rmr 170 Apr 6 22:46 s1 
drwxrwxrwx 4 rmr rmr 136 Oct 62005 s10

drwxrwxrwx 5 rmr rmr 170 Apr 6 22:50 s2

drwxrwxrwx $5 \mathrm{rmr}$ rmr 170 Oct 62005 s3

drwxrwxrwx 5 rmr rmr 170 Oct 62005 s 4

drwxrwxrwx 4 rmr rmr 136 Oct 62005 s5

drwxrwxrwx 5 rmr rmr 170 Dec 30 07:17 s6

drwxrwxrwx 4 rmr rmr 136 Oct 62005 s7

drwxrwxrwx 5 rmr rmr 170 Oct 62005 s8

drwxrwxrwx 4 rmr rmr 136 Oct 62005 s9

FOLDER /Users/rmr/data/mid/level1/iop4:

total 16

-rw-r--r-- 1 rmr rmr 542 Apr 6 22:47 INFO.tmp

-rwxrwxrwx $1 \mathrm{rmr}$ rmr 775 Sep 212005 INFO_mid_iop4.txt

drwxrwxrwx $5 \mathrm{rmr}$ rmr 170 Oct 62005 s1

drwxrwxrwx 4 rmr rmr 136 Oct 62005 s10

drwxrwxrwx 5 rmr rmr 170 Oct 62005 s11

drwxrwxrwx 5 rmr rmr 170 Oct 62005 s12

drwxrwxrwx 4 rmr rmr 136 Oct 62005 s3

drwxrwxrwx 4 rmr rmr 136 Oct 62005 s 4

drwxrwxrwx 4 rmr rmr 136 Oct 62005 s5

drwxrwxrwx 4 rmr rmr 136 Oct 62005 s7

drwxrwxrwx $5 \mathrm{rmr}$ rmr 170 Oct 62005 s8

drwxrwxrwx 4 rmr rmr 136 Oct 62005 s9

FOLDER /Users/rmr/data/mid/level1/iop5:

total 16

-rw-r--r-- 1 rmr rmr 539 Apr 6 22:48 INFO.tmp

-rwXrwxrwx 1 rmr rmr 541 Sep 212005 INFO_mid_iop5.txt

drwxrwxrwx $5 \mathrm{rmr}$ rmr 170 Oct 6 20:02 s1

drwxrwxrwx 4 rmr rmr 136 Oct 62005 s10

drwxrwxrwx 4 rmr rmr 136 Oct 62005 s11

drwxrwxrwx 5 rmr rmr 170 Oct 62005 s12

drwxrwxrwx $4 \mathrm{rmr}$ rmr 136 Oct 62005 s3

drwxrwxrwx 4 rmr rmr 136 Oct 62005 s4

drwxrwxrwx 4 rmr rmr 136 Oct 62005 s5

drwxrwxrwx 5 rmr rmr 170 Oct 62005 s7

drwxrwxrwx $5 \mathrm{rmr}$ rmr 170 Oct 62005 s8

drwxrwxrwx 4 rmr rmr 136 Oct 62005 s9

FOLDER /Users/rmr/data/mid/level1/iop6:

total 16

-rw-r--r-- 1 rmr rmr 539 Apr 6 22:49 INFO.tmp

-rwxrwxrwx 1 rmr rmr 785 Sep 212005 INFO_mid_iop6.txt

drwxrwxrwx 5 rmr rmr 170 Oct 62005 s1

drwxrwxrwx 4 rmr rmr 136 Oct 62005 s10 
drwxrwxrwx 4 rmr rmr 136 Oct 62005 s11

drwxrwxrwx 5 rmr rmr 170 Oct 62005 s12

drwxrwxrwx 4 rmr rmr 136 Oct 62005 s3

drwxrwxrwx 4 rmr rmr 136 Oct 62005 s4

drwxrwxrwx 4 rmr rmr 136 Oct 62005 s5

drwxrwxrwx 4 rmr rmr 136 Oct 62005 s7

drwxrwxrwx $5 \mathrm{rmr}$ rmr 170 Oct 62005 s8

drwxrwxrwx 4 rmr rmr 136 Oct 62005 s9

FOLDER /Users/rmr/data/mid/level1/meso:

total 16

-rw-r--r-- 1 rmr rmr 695 Apr 7 00:07 INFO.tmp

-rwxrwxrwx $1 \mathrm{rmr}$ rmr 591 Oct 12005 INFO_mid_meso.txt

drwxr-xr-x 4 rmr rmr 136 Apr 6 23:05 ccny_roof_met

drwxrwxrwx 6 rmr rmr 204 Apr 6 23:25 cpk_CentralPark_asos

drwxr-xr-x 5 rmr rmr 170 Apr 6 23:07 eml_EnvironMeasLab_DCnet

drwxr-xr-x 6 rmr rmr 204 Apr 6 23:25 ewr_Newark_asos

drwxrwxrwx 7 rmr rmr 238 Apr 14 06:25 gm1_GeneralMotors_met

drwxrwxrwx 42 rmr rmr 1428 Apr 14 06:26 gm2_GMBldg_sodar

drwxr-xr-x 6 rmr rmr 204 Apr 6 23:26 jfk_Kennedy_asos

drwxrwxrwx 4 rmr rmr 136 Apr 6 23:28 lbr1_LhrBros_roof_noaa

drwxrwxrwx $5 \mathrm{rmr}$ rmr 170 Oct 62005 lbr2_LehmanBros_setback

drwxrwxrwx 6 rmr rmr 204 Apr 6 23:29 lga_LaguardiaAP_asos

drwxrwxrwx 7 rmr rmr 238 Apr 14 06:26 met1_MetLife_met

drwxrwxrwx $42 \mathrm{rmr}$ rmr 1428 Apr 14 06:27 met2_MetLife_sodar

drwxrwxrwx $5 \mathrm{rmr}$ rmr 170 Oct 42005 mgh1_McGraw-Hill_roof

drwxrwxrwx $5 \mathrm{rmr}$ rmr 170 Oct 52005 mgh2_McGraw-Hill_setback

drwxrwxrwx $5 \mathrm{rmr}$ rmr 170 Oct 62005 opp

drwxrwxrwx $5 \mathrm{rmr}$ rmr 170 Oct 62005 ppz_ParkPlaza_roof

drwxr-xr-x 7 rmr rmr 238 Apr $623: 53$ sit1_met 


\section{Appendix C: RM Young 81000 UltraSonic Anemometer}

\section{YOUNG}

\section{Model 81000 Ultrasonic Anemometer}

The YOUNG Model 81000 Ultrasonic Anemometer is a 3-axis, no moving parts wind sensor. It is perfectly suited for applications requiring fast response, high resolution and threedimensional wind measurement.

The sensor features durable, corrosion-resistant construction with 3 opposing pairs of ultrasonic transducers supported by stainless steel members. The transducers are arranged so that measurements are made through a common volume. A fast, 160 $\mathrm{Hz}$ internal sampling rate ensures superior measurement resolution. Output rates from 4 to $32 \mathrm{~Hz}$

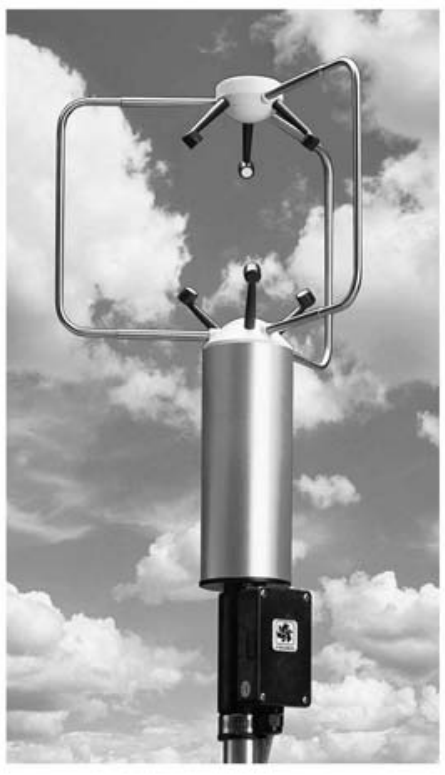
may be selected. Each 81000 is individually wind-tunnel tested and calibrated to compensate for wind shadow effects of the support structure.

Model $\mathbf{8 1 0 0 0}$ features four voltage ouput channels. Serial RS-232 and RS-485 outputs are available as well. For applications requiring synchronized analog measurements, Model $81000 \mathrm{~V}$ includes four voltage input channels instead of voltage outputs. Wind, sonic temperature and voltage input data are transmitted serially. For each model, a variety of preset or custom output format options may be selected by the user.

Both models install on standard 1 inch pipe. Wiring connections are housed in a convenient weatherproof junction box.

Ordering Information MODEL

ULTRASONIC ANEMOMETER- VOLTAGE \& SERIAL OUTPUTS 81000 ULTRASONIC ANEMOMETER- VOLTAGE INPUTS, SERIAL OUTPUTS ONLY $\ldots \ldots . .81000 \mathrm{~V}$

\section{Specifications}

Wind Speed: 0 to $40 \mathrm{~m} / \mathrm{s}$ (0 to $90 \mathrm{mph})$ Resolution: $0.01 \mathrm{~m} / \mathrm{s}$

Threshold: $0.01 \mathrm{~m} / \mathrm{s}$

Accuracy: $\pm 1 \% \mathrm{rms} \pm 0.05 \mathrm{~m} / \mathrm{s}(0$ to $30 \mathrm{~m} / \mathrm{s})$

$\pm 3 \% \mathrm{rms}(30$ to $40 \mathrm{~m} / \mathrm{s})$

Wind Direction: 0 to 360 degrees

Elevation Range: \pm 60 degrees

Resolution: 0.1 degree

Accuracy: \pm 2 degrees (1 to $30 \mathrm{~m} / \mathrm{s}$ )

\pm 5 degrees $(30$ to $40 \mathrm{~m} / \mathrm{s}$ )

Speed of Sound: 300 to $360 \mathrm{~m} / \mathrm{s}$

Resolution: $0.01 \mathrm{~m} / \mathrm{s}$

Accuracy: $\pm 0.1 \% \mathrm{rms} \pm 0.05 \mathrm{~m} / \mathrm{s}(0$ to $30 \mathrm{~m} / \mathrm{s})$

Sonic Temperature: -50 to $+50^{\circ} \mathrm{C}$

Resolution: $0.01{ }^{\circ} \mathrm{C}$

Accuracy: $\pm 2^{\circ} \mathrm{C}(0$ to $30 \mathrm{~m} / \mathrm{s})$

\section{Serial Output:}

RS-232 or RS-485

1200 to 38400 baud

4 to $32 \mathrm{~Hz}$ (user-selected)

User Programmable ASCII output configuration

(select from U, V, W, Speed of sound, Sonic

temperature, $2 \mathrm{D}$ speed, $3 \mathrm{D}$ speed, Azimuth,

Elevation)

Preset outputs:

NMEA-Marine Standard

RMYT- Young Wind Tracker

Units: $\mathrm{m} / \mathrm{s}, \mathrm{cm} / \mathrm{s}, \mathrm{MPH}, \mathrm{Knots}, \mathrm{Km} / \mathrm{hr}$

Analog Voltage Outputs (81000):

4 voltage outputs, 0 to $5000 \mathrm{mV}$

(select from U, V. W. Sonic temperature or

Speed, Azimuth, Elevation, Sonic temperature)

Voltage Input (81000V):

Range: 0 to $5000 \mathrm{mV}$, V1 \& V2

0 to $1000 \mathrm{mV}, \mathrm{V} 3 \&$ V 4

Resolution: 1 part in 4000

Accuracy: $\pm 0.1 \%$ of full scale

Power Requirement:

12 to 24 VDC, $110 \mathrm{~mA}$

\section{Operating Temperature:}

-50 to $+50^{\circ} \mathrm{C}$

Dimensions:

$56 \mathrm{~cm}$ high $\times 17 \mathrm{~cm}$ radius ( 3 support arms)

Weight: $1.7 \mathrm{~kg}(3.8 \mathrm{lb})$

Shipping Weight: $4.5 \mathrm{~kg}(10 \mathrm{lb})$

\section{$c \epsilon$}

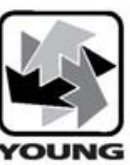

R.M. YOUNG COMPANY

2801 Aero Park Drive

Traverse City, Michigan 49686 USA

TEL: (231) 946-3980 FAX: (231) 946-4772

E-mail: met.sales@youngusa.com

Web Site: www.youngusa.com 
84 of 88 